This work reports on laterally resolved measurements of the current-induced gradient in the electrochemical potential of multiwall carbon nanotubes. Nanotubes with different classes of defects were studied at room temperature. The potential profile of the outermost shell peng the the was measured in a local as well as in along the tube was a nonlocal geometry. The da have been used to separate the contributions of various shells to the total resistance of the whole tube. For this purpose, a classical resistivity model was used that describes the measured potentia profiles well. Additionally, the influence of structural defects on the conductivity has been quantified. Particularly, defects such as an ending outermost shell, an a kink were investigated.

Additionaly, measuredents at low temperaures re-

Additionally, measurements

vealed some quantum conductance effects, such as

weak localization and oscillations in the potential pro-

file. The latter could be traced back to the same origin

as the universal conductance fluctuations.

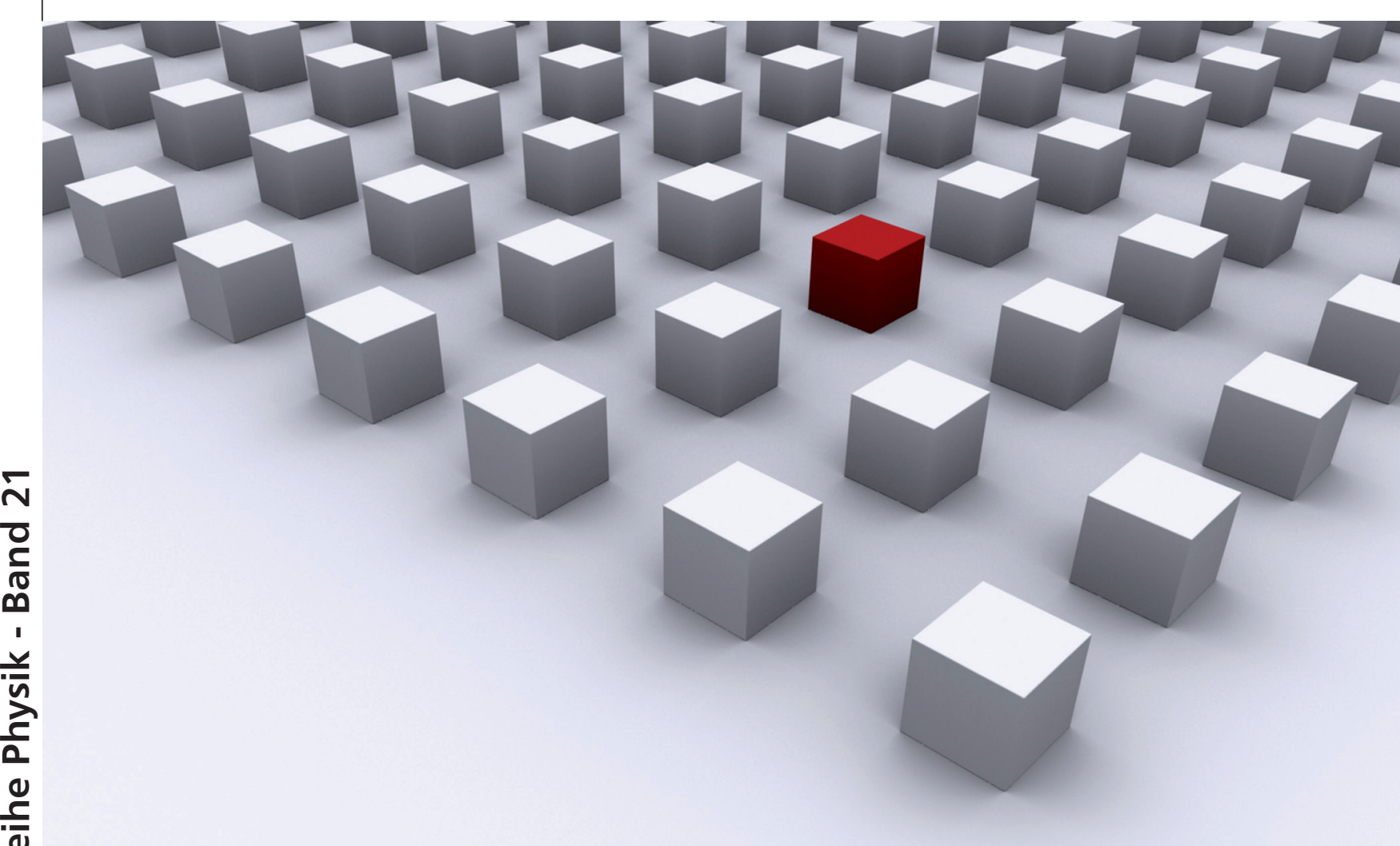

\title{
Andreas Stetter
}

Conductivity of Multiwall

Carbon Nanotubes: Role of

Multiple Shells and Defects
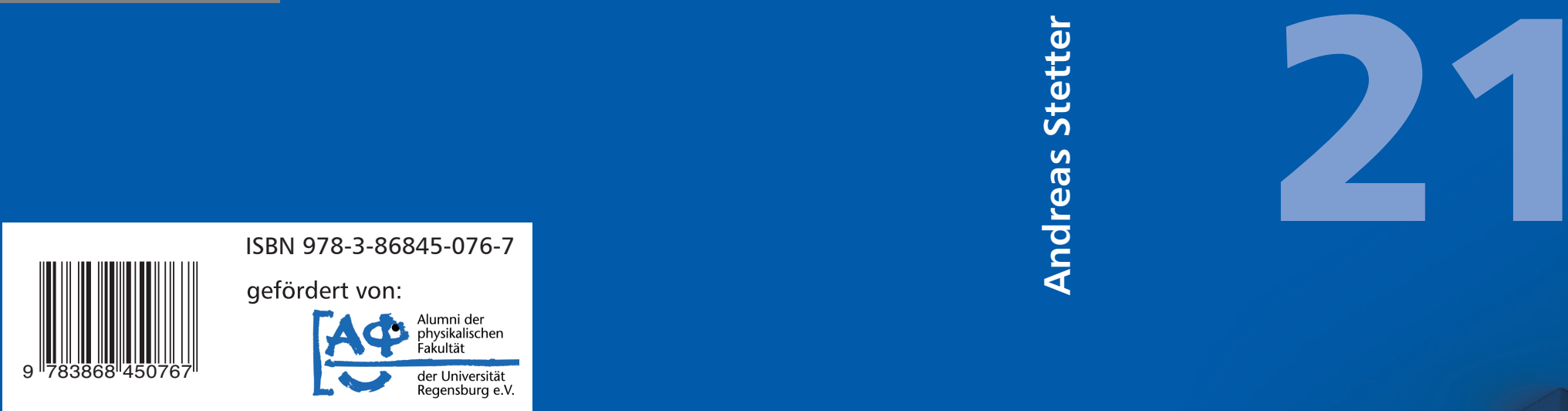

Universitätsverlag Regensburg

QR

Universität Regensburg

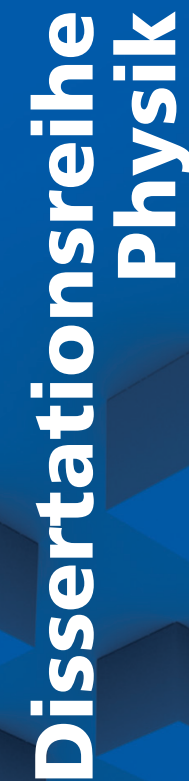


Andreas Stetter

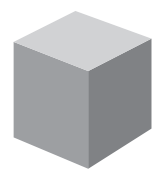

Conductivity of Multiwall

Carbon Nanotubes: Role of Multiple Shells and Defects 


\section{Conductivity of Multiwall Carbon Nanotubes:} Role of Multiple Shells and Defects

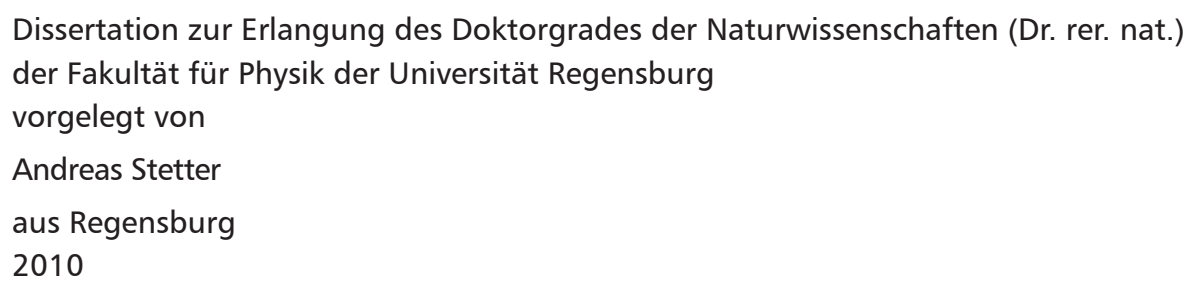

Die Arbeit wurde von Prof. Dr. C. H. Back angeleitet.

Das Promotionsgesuch wurde am 29.10.2010 eingereicht.

Prüfungsausschuss: Vorsitzende: Prof. Dr. M. Grifoni

1. Gutachter: Prof. Dr. C. H. Back

2. Gutachter: Prof. Dr. C. Strunk weiterer Prüfer: Prof. Dr. R. Huber

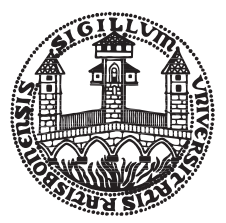

Dissertationsreihe der Fakultät für Physik der Universität Regensburg, Band 21

Herausgegeben vom Präsidium des Alumnivereins der Physikalischen Fakultät:

Klaus Richter, Andreas Schäfer, Werner Wegscheider, Dieter Weiss 
Andreas Stetter

Conductivity of Multiwall

Carbon Nanotubes: Role of

Multiple Shells and defects 
Bibliografische Informationen der Deutschen Bibliothek.

Die Deutsche Bibliothek verzeichnet diese Publikation

in der Deutschen Nationalbibliografie. Detailierte bibliografische Daten sind im Internet über http://dnb.ddb.de abrufbar.

1. Auflage 2011

(C) 2011 Universitätsverlag, Regensburg

Leibnizstraße 13, 93055 Regensburg

Konzeption: Thomas Geiger

Umschlagentwurf: Franz Stadler, Designcooperative Nittenau eG

Layout: Andreas Stetter

Druck: Docupoint, Magdeburg

ISBN: 978-3-86845-076-7

Alle Rechte vorbehalten. Ohne ausdrückliche Genehmigung des Verlags ist es nicht gestattet, dieses Buch oder Teile daraus auf fototechnischem oder elektronischem Weg zu vervielfältigen.

Weitere Informationen zum Verlagsprogramm erhalten Sie unter:

www.univerlag-regensburg.de 


\section{Conductivity of Multiwall Carbon Nanotubes: \\ Role of Multiple Shells and Defects}

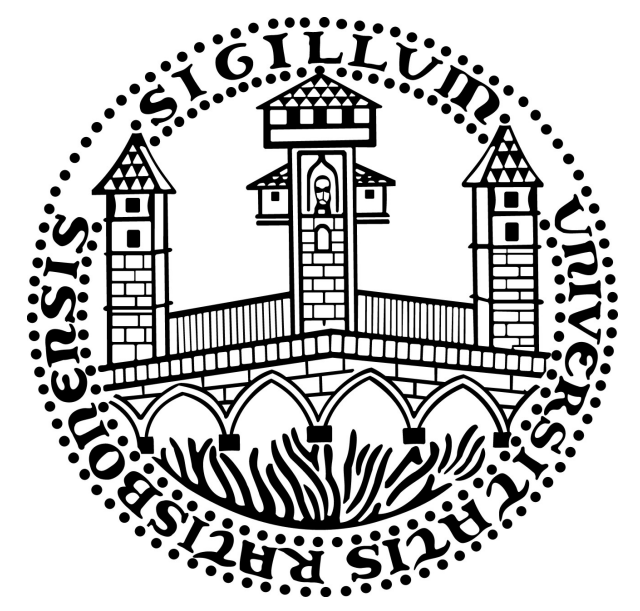

DisSERTATION

ZUR ERLANGUng Des DoKTORgRAdes DER NATURWISSENSCHAFTEN

(DR. RER. NAT.)

DeR FAKUltät Physik DeR Universität

REgensBurg

vorgelegt von

Andreas Stetter

aus Regensburg

im Jahr 2010 
Promotionsgesuch eingereicht am 29.10.2010

Die Arbeit wurde angeleitet von: Prof. Dr. C. H. Back

\section{Prüfungsausschuss:}

Vorsitzende: Prof. Dr. M. Grifoni

1. Gutachter: Prof. Dr. C. H. Back

2. Gutachter: Prof. Dr. C. Strunk

Weiterer Prüfer: Prof. Dr. R. Huber 


\section{Contents}

List of Figures $\quad$ VII

1 A History of carbon science 1

2 Carbon nanotubes: A one dimensional material 3

2.1 Structure of carbon nanotubes . . . . . . . . . . . . . . 3

2.1.1 Ideal carbon nanotubes . . . . . . . . . . . . . . . . . . . . . . . . . . . . . . .

2.1.2 The reality: dirt and defects . . . . . . . . . . . . 5

2.2 Bandstructure and density of states . . . . . . . . . . . . 8

2.2.1 Graphene: the basis for nanotubes . . . . . . . . . 8

2.2.2 Carbon nanotubes: graphene rolled up to a cylinder . . . . 8

2.3 Transport properties . . . . . . . . . . . . . . . . . . . 11

2.3.1 Quantum conductance .............. . . 11

2.3.2 Conductance at room temperature . . . . . . . . . . 13

2.3.3 The role of defects and multiple shells . . . . . . . . . . 14

3 The resistance network model of a MWCNT 17

3.1 Punctual current injection in an infinitely long tube . . . . . . . . 17

3.1.1 Beyond the electrodes . . . . . . . . . . . . . . . 18

3.1.2 Between the electrodes . . . . . . . . . . . . . . . . 19

3.1 .3 Discussion . . . . . . . . . . . . . . . 20

3.2 Continuous current injection and finite tube length . . . . . . . . 21

3.2 .1 Injection zone . . . . . . . . . . . . . . . . . . . . . . . . . . 22

3.2 .2 Boundary conditions . . . . . . . . . . . . . . . . 23

3.2.3 Discussion . . . . . . . . . . . . . . . . 25

3.3 Comparison of both models . . . . . . . . . . . . 27

4 Experimental Setup $\quad 29$

4.1 Sample design . . . . . . . . . . . . . . . . . . . . . . . . . . . . . . . . . . . 30

4.2 Measurement setup . . . . . . . . . . . . . . . . 32 
5 Results obtained at room temperature $\quad 37$

5.1 Sample A: a multiwall carbon nanotube with no obvious defects . 38

5.2 Sample B: a multiwall carbon nanotube with an incomplete outermost shell . . . . . . . . . . . . . . . . . . . 45

5.3 Sample C: A tube with a strongly varying diameter . . . . . . . . 49

5.3.1 Characteristics without gate voltage . . . . . . . . . 49

5.3 .2 Behavior with applied gate voltage . . . . . . . . . . 52

5.4 Sample D: a stretched nanotube with a kink . . . . . . . . . . 53

6 Low temperature results $\quad 59$

6.1 Measurements in local geometry . . . . . . . . . . . . . . . . 60

6.2 Measurements in non-local geometry . . . . . . . . . . . . 63

$\begin{array}{lll}7 & \text { Summary } & 69\end{array}$

$\begin{array}{ll}\text { Bibliography } & 71\end{array}$ 


\section{List of Figures}

2.1 Structure and chiral vector of carbon nanotubes . . . . . . . . 4

2.2 Defects in the hexagonal lattice . . . . . . . . . . . . 5

2.3 Edge dislocation in a multiwall carbon nanotube . . . . . . . . 6

2.4 Bent Nanotubes . . . . . . . . . . . . . . . . . . . . . 7

2.5 Energy level of $\sigma$ and $\pi$ bonds . . . . . . . . . . . . . . . 7

$2.6 \pi$ and $\sigma$ band relative to the Fermi level . . . . . . . . . . . . 9

2.7 Band structure of graphene with allowed $\mathbf{k}$ vectors . . . . . . . . . 10

2.8 Dispersion relation and density of states . . . . . . . . . . . . 10

3.1 Resistor network of a double-wall nanotube . . . . . . . . . . . . 18

3.2 Current leaving the region between the electrodes . . . . . . . . . 20

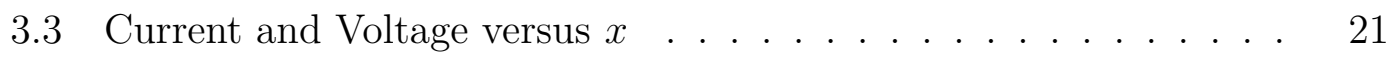

3.4 Resistor model with injection zone . . . . . . . . . . . . 22

3.5 Effect of $l_{l}$ on the current . . . . . . . . . . . . . . . 25

3.6 Current in the injection zone . . . . . . . . . . . . . 26

3.7 Effect of $l_{l}$ on the potential profile . . . . . . . . . . 27

3.8 Comparison of current and voltage with and without additional electrode . . . . . . . . . . . . . . . . . 28

4.1 Tunneling characteristics of the insulating layer . . . . . . . . . 31

4.2 Schematic drawing of the sample . . . . . . . . . . . . . . . . . . . 32

4.3 The UHV-Nanoprobe . . . . . . . . . . . . . . . . . . . . . . 33

4.4 The measurement setup .................. 34

5.1 Carbon nanotube with and without electrodes . . . . . . . . . 38

5.2 STM image of the parts between and beyond the electrodes . . . . 39

5.3 Potential profile of sample A . . . . . . . . . . . . . . . 40

5.4 Influence of the tube length on the bending . . . . . . . . . . . . 41

5.5 Comparison of the potential decrease in the inner and the outermost

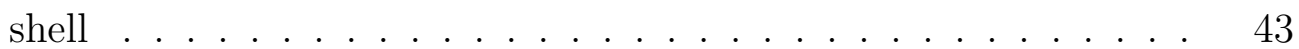


5.6 Illustration and measurement of a tube with incomplete outermost

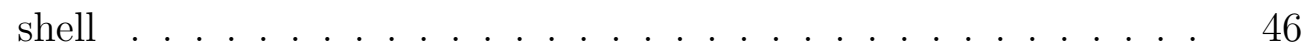

5.7 Potential measurements for both current directions . . . . . . . . 47

5.8 Model with incomplete outermost shell . . . . . . . . . . . . . . . 48

5.9 SEM image of sample $\mathrm{C}$ with varying radius . . . . . . . . . . . 50

5.10 Potential profile of sample $\mathrm{C}$ without applied gate voltage . . . . 51

5.11 Potential profiles of sample $\mathrm{C}$ with applied gate voltage . . . . . . 52

5.12 Dependency on the gate voltage at one position . . . . . . . . 52

5.13 Sample D before and after manipulation . . . . . . . . . . 53

5.14 Potential profile of sample D with kink . . . . . . . . . . . 54

5.15 Non-local potential profile of sample D . . . . . . . . . . . . 57

6.1 Local low temperature potential profiles of sample D . . . . . . 60

6.2 Conductivities of the three sectors as a function of temperature . 62

6.3 Non-local potential profile of sample D at $T=110 \mathrm{~K} \ldots \ldots 4$

6.4 Non-local potential profile of sample D at $T=28 \mathrm{~K}$. . . . . . . . 64

6.5 Interference between different pathways . . . . . . . . . . . 68 


\section{A History of carbon science}

For a long time, carbon in its elemental state had been thought to exist in only three configurations. In its amorphous state, e.g. in charcoal and coal, carbon contributed to early technology of mankind. Early metallurgy is hardly imaginable without the use of charcoal for heating and reducing metal ores. Later coal and its derivative coke along with new technology initiated the industrial revolution. Another application for amorphous carbon that is used since the antiquity is carbon black, a pure form of soot. Soot will be even more interesting further below in this chapter.

A second form of elemental carbon is graphite. It was used for writing and drawing in form of a pencil as well as for crucibles for molten steel because of its heat resistance. Due to this, combined with the weak bond between single layers, graphite is a good lubricant for components that have to withstand high temperatures. Graphite is a stack of hexagonal graphene layers (this name was proposed by ref. [1]) in $\mathrm{AB}$ configuration. In order to achieve the planar hexagonal lattice the carbon atoms are $s p^{2}$ hybridized, where the three $\sigma$-bonds connect the adjacent atoms in an angle of $120^{\circ}$. The $\pi$-orbitals, however, combine to a charge cloud below and above the plain.

The third elemental form of carbon, diamond, was also known since the antiquity but only used as jewelery for a long time. Since diamond is the hardest material known, its applications for tools are countless. Furthermore, the thermal conductivity of diamond is about five times higher than that of silver, although it is a perfect electrical insulator. These extraordinary properties result from the hard $\sigma$-bonds between the $s p^{3}$ hybridized carbon atoms in tetrahedral configuration.

1967 a fourth manifestation of carbon was described: Lonsdalite also called hexagonal diamond [2]. It is created from graphite under high pressure and high temperatures where the orbital hybridization changes from $s p^{2}$ to $s p^{3}$ while the hexagonal configuration is kept.

The next step in carbon science was in 1985 as Kroto et al. [3] synthesized remarkably stable pure carbon molecules consisting of 60 atoms. They proposed 
a round structure with $s p^{2}$ hybridized hexagons and pentagons, arranged similarly to a football and called them Buckminsterfullerenes. Subsequently small closed carbon nanostructures like $\mathrm{C}_{60}$ or $\mathrm{C}_{70}$ became a large domain in science once they could easily be produced. For this purpose Krätschmer et al. [4] vaporized graphite in an arc in helium atmosphere and washed the fullerenes out of the formed soot.

In 1991 Iijima [5], who had already investigated soot in a transmission electron microscope and found graphitized carbon nanoparticles ten years earlier took some of the junk from the cathode of such an arc arrangement and found long hollow fibers with several walls [6]. This multiwall carbon nanotubes (MWCNTs) were formed of several spheric fullerenes cut in two and connected again with a rolled up layer of graphite (graphene), one inside the other like a Matryoshka doll. In practice, the caps have mostly much more complex structures. The nanotubes ranged in length from a few tens of nm up to several $\mu \mathrm{m}$ and a diameter of 4 to $30 \mathrm{~nm}$. The innermost tubes had diameters of about 2 to $4 \mathrm{~nm}$.

Soon two groups reported independently the synthesis of single-walled carbon nanotubes. One was again Iijima, this time together with Ichihashi [7], of NEC and the other were Bethune et al. [8] from the IBM Research Division. They both used a quite similar apparatus like for MWCNTs but contaminated their arc electrodes with iron and cobalt, respectively. This was an important development in order to describe experiments with theory which is reliably for the more simple single-wall tubes. Further investigations led to additional synthesis methods like laser vaporisation [9] or catalytic methods [10].

Eventually, three dimensional (diamond, graphite), one dimensional (carbon nanotubes) and zero dimensional (spherical fullerenes) carbon structures were established. Two dimensional structures, however, were presumed virtually impossible due to existing theorems [11]. 2004 Novoselov [12] surprised with preparing free standing single layer graphene on insulating $\mathrm{SiO}_{2}$. One year later the prediction that electrons behave like massless dirac fermions [13] has been confirmed [14, 15]. Since then graphene has drawn at least as much attention in science as carbon nanotubes or fullerenes. 


\section{Carbon nanotubes: A one dimensional material}

The first micrographs of multiwall carbon nanotubes were published in a Russian journal [16], nearly forty years before the modern nanotube research was triggered by Iijima [6] in 1991. The high resolution transmission electron micrographs (TEM) of Iijima are nevertheless the first ones that revealed the multiple shell configuration of the multiwall carbon nanotubes (MWCNT).

\subsection{Structure of carbon nanotubes}

\subsubsection{Ideal carbon nanotubes}

The structure of a single-wall carbon nanotube (SWCNT) is easy imaginable by cutting a rectangle out of a graphene sheet and connecting two sides of it by rolling it up to a hollow cylinder. The connection condition of the crystal lattice allows only rectangles with the same configuration on two opposing sides. For easier notation the chiral vector $\overrightarrow{C_{h}}$ was adopted (see upper left of fig. 2.1), that corresponds to the circumference of the tube. If $m=0\left(\Theta=0^{\circ}\right)$ it results a zigzag tube and for $n=m\left(\Theta=30^{\circ}\right)$ it results an armchair tube referring to the structure along the circumference. All other combinations result in so-called chiral tubes (illustrations in fig. 2.1. To saturate the dangling bonds on the end of the tube they are capped with an attached hemispherical fullerene. Since the circumference is the absolute value of $\vec{C}_{h}$, the diameter can be calculated to:

$$
d_{T}=\frac{\left|\vec{C}_{h}\right|}{\pi}=\frac{a}{\pi} \sqrt{n^{2}+n m+m^{2}}
$$

where $a=4.46 \AA$ is the lattice constant of the graphene honeycomb structure. The diameters of real single-wall carbon nanotubes spread from about $0.4 \mathrm{~nm}$ [18] 


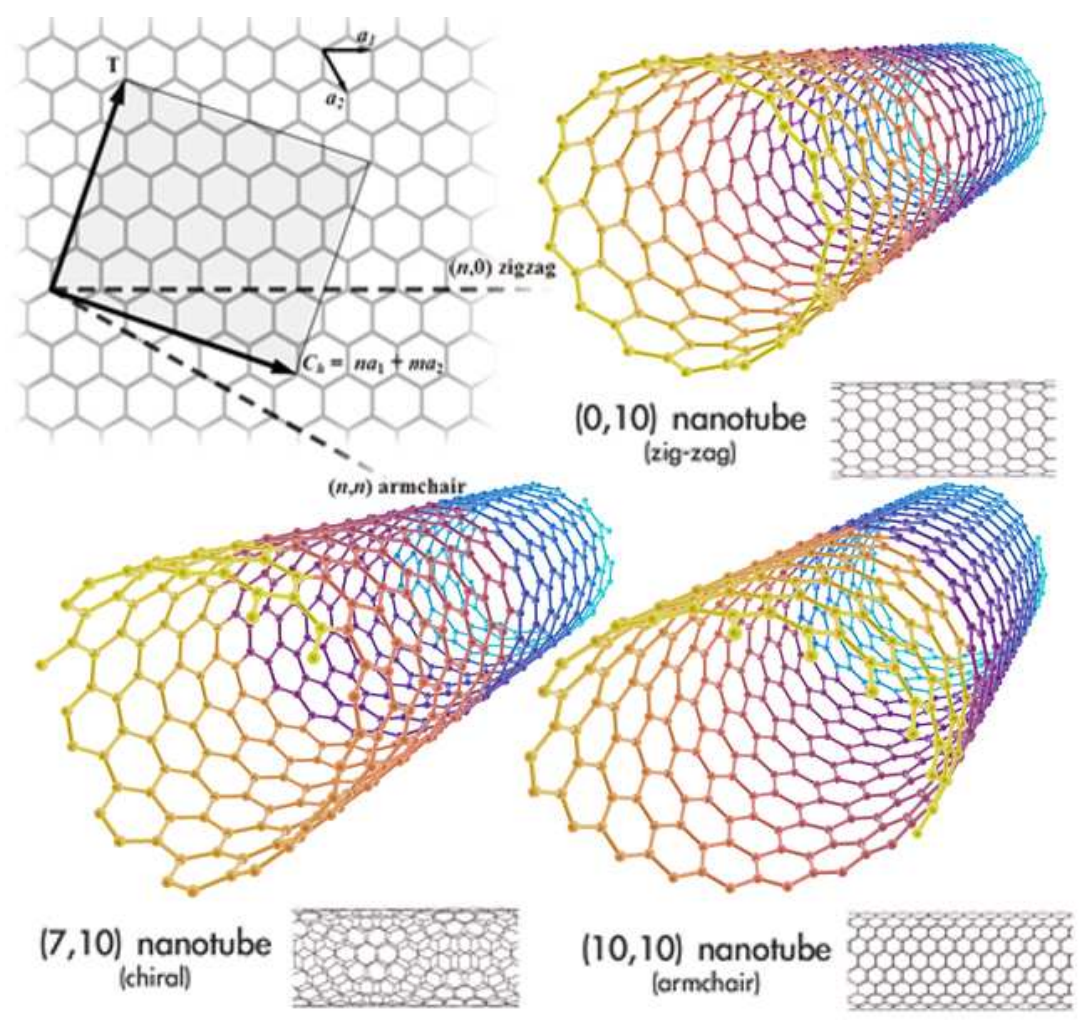

Figure 2.1: The chiral vector $\overrightarrow{C_{h}}=n \overrightarrow{a_{1}}+m \overrightarrow{a_{2}}$ (see upper left part of the image) points from one K-point to an equivalent one of another cell. If one moves an integer number $n$ of lattice vector $\overrightarrow{a_{1}}$ and $m$ of vector $\overrightarrow{a_{2}}$ (in this example 4 and 2 , respectively) this condition is fulfilled. The angle between the two lattice vectors $\overrightarrow{a_{1}}$ and $\overrightarrow{a_{2}}$ is $60^{\circ}$. $\Theta$ is the chiral angle and $(\mathrm{n}, \mathrm{m})$ are the chiral indices. The sheet is joined along $\vec{T}$ that has the direction axial to the tube. The other parts illustrate the structures of zig-zag, armchair and chiral tubes [17]. 
(a)

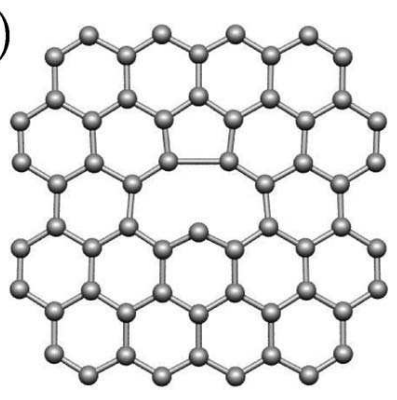

(b)

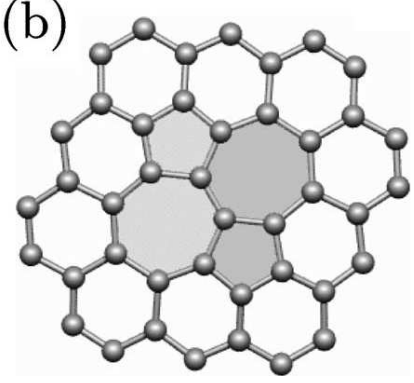

(c)

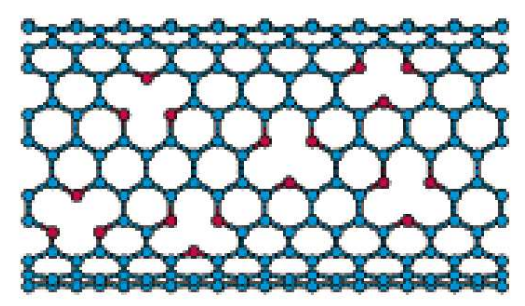

Figure 2.2: (a) Graphene fragment containing a vacancy. The carbon network exhibits some reconstruction. (b) Graphene lattice with a Stone-Wales defect. (c) Nanotube doped with Nitrogen. Adapted from [24] ((a) and (b)) and [25] ((c)).

up to $6 \mathrm{~nm}$ (e.g.[19, 20]). Above this diameter SWCNTs are predicted to collapse [21]. Generally the chiral angles are evenly distributed [22].

Multiwall carbon nanotubes consist of several shells each of them looking like a single-wall nanotube. The differences in the radii are in the range of $0.34 \mathrm{~nm}$, similar to the interlayer distance in graphite [6]. Due to the difference in the diameters and therefore also to the different chiral vector, the individual layers are incommensurate. So the stacking of the layers differs necessarily from that of graphite whereby many attributes like interlayer conductance is not comparable anymore.

\subsubsection{The reality: dirt and defects}

Nowadays three techniques dominate CNT production: chemical vapor deposition [10], arc discharge [6] and laser ablation [9]. Although big progress has been made in this area, CNTs always appear together with amorphous soot and other carbonaceous contamination. Additionally, for singe-wall tubes a metallic catalyst is needed, that also gives rise to metallic impurities or to a compound of metal with carbon. Since for technical or scientific applications clean nanotubes are desirable, purification methods are needed. These range from chemical oxidation, filtration and centrifugation to solubilization with functional groups and annealing. For an overview see ref. [23].

Just as the environment of as-grown nanotubes is not perfect so are the tubes themselves. For single-wall nanotubes there are mainly defects in the hexagonal lattice like vacancy, substitution of carbon atoms or including heptagons and pentagons in the lattice (fig. 2.2). One of the most common defects is the Stone-Wales 


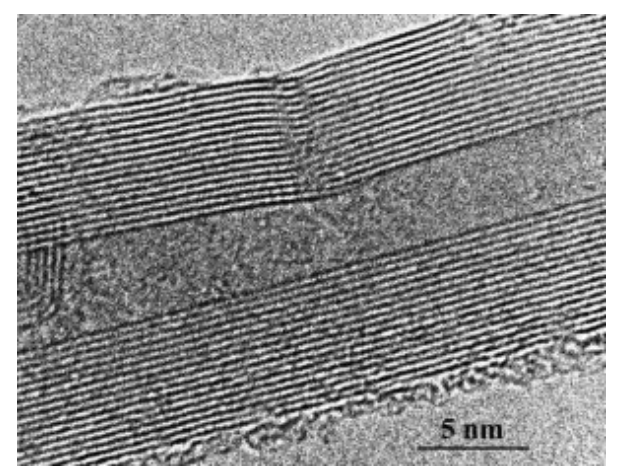

Figure 2.3: Edge dislocation in a multiwall carbon nanotube. This should be a change-over from a nested to a scrolled nanotube. Adapted from [29]

defect [26], where only one covalent bond rotates with $90^{\circ}$, and two pentagons as well as two heptagons are generated from the original hexagonal structure. Generally, individual pentagons or heptagons introduce convex and concave bending of the tube, respectively. Furthermore heptagons and pentagons can allow a connection between tubes with different diameters and chirality [27].

In multiwall carbon nanotubes additional defects due to interlayer effects can appear. In high resolution TEM images of MWCNTs it was quite often observed that the distances between the fringes on both sides of the tube differ from each other. Consequently the interlayer distance cannot be constant. Liuand et al. [28] observed a polygonal cross section and suggested the existence of several nearplanar regions. These are joined together along lines of small radius of curvature. It was debated if the stacking in the planar regions is similar to graphite.

Especially the inner structures of MWCNTs can vary significantly. There can be one or more layers traversing the central core. Sometimes even closed compartments are seen [30]. Edge dislocations, for example, are defects affecting all shells. At this defect on one side of the tube the outermost shell is connected with the second outer shell and so on (fig. 2.3). It was discussed that this defect can represent a change-over from a nested (as described above) to a scroll type nanotube ${ }^{1}$ [29]. Intershell connections can also be induced artificially by breaking bonds via ozone exposure. The atoms rearrange creating $s p^{3}$ orbitals and cross-link the shells [31]. This may lead to a increased transmission probability between the shells.

The Stone-Wales defects may also be induced by mechanical strain [32, 33]. Applying additional strain and depending on chirality and temperature, the heptagons

\footnotetext{
${ }^{1}$ Although it is not easy to distinguish a nested from a scroll type nanotube with TEM, there are several indications that the nested structure is at least the more common: HR-TEM images show the same number of shells on both sides. The observed caps and internal closed compartments are difficult to explain in a scroll structure; at least reactive gases attack the cap region preferentially, i.e. the tube itself has no specific area of attack.
} 


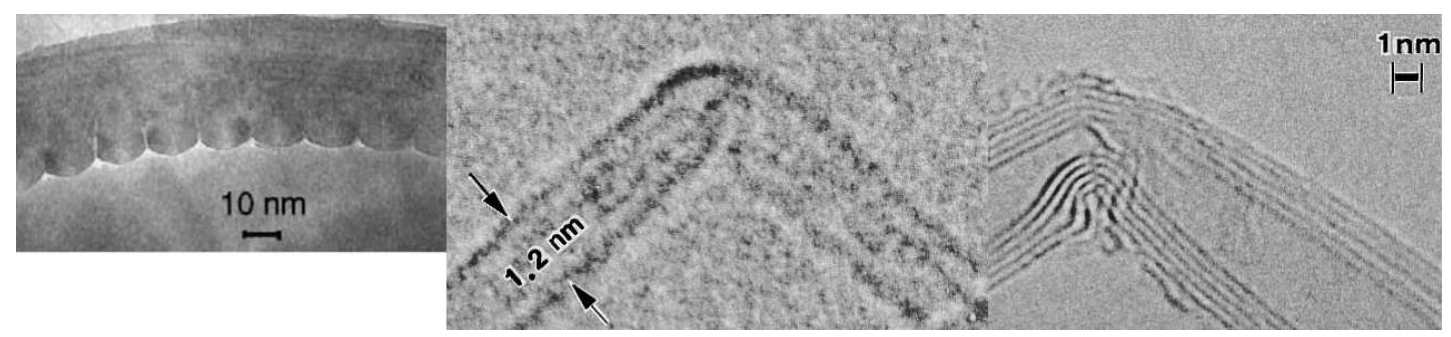

Figure 2.4: HR-TEM images of slightly bent (left) (adapted from [38]) and strongly bent (right) nanotubes (adapted from [39]).

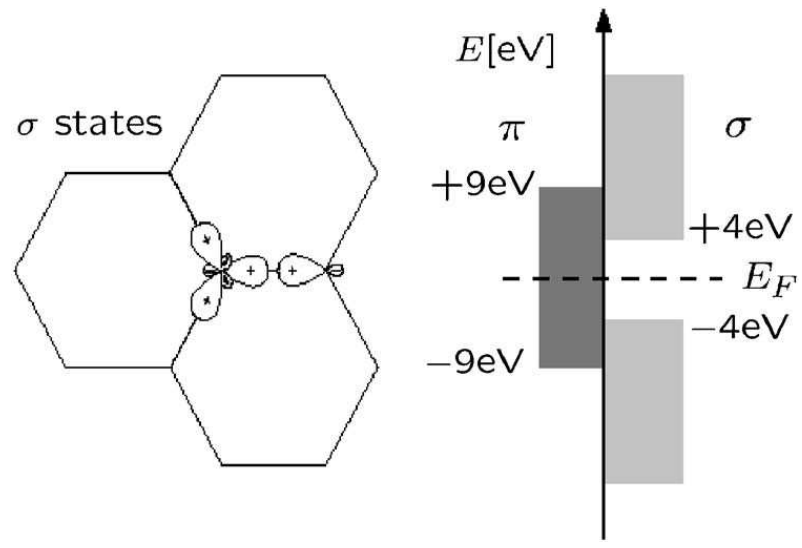

Figure 2.5: The $\sigma$-bonds connect the carbon atoms to the characteristic hexagonal lattice and are responsible for the binding energy and the elastic properties (left). The corresponding bands are separated by a large energy gap, whereas the energy level of the $\pi$ orbitals lies around the Fermi level $E_{F}$ (right). Adapted from [40]

and pentagons can diverge, again via bond rotations, moving around and along the tube leaving a slightly thinner tube with another chirality [33, 34]. Other tubes can become brittle due to many heptagon, pentagon or octagon defects [34], or the molecular bonds can fracture individually [35]. Especially for multiwall tubes there is another possibility to release the strain: individual tubes can break at different positions and the whole tube can be extended telescopically [36, 37].

Another possibility to get a kink, besides including heptagons or pentagons while growing is bending the originally ideal nanotube with mechanical force. The resulting structure depends on the radius of curvature. Generally, the structure of the outer side remains flat, while the inner side buckles. Examples for slightly and strongly bent tubes are given in fig. 2.4. 


\subsection{Bandstructure and density of states}

\subsubsection{Graphene: the basis for nanotubes}

In all graphene based structures (graphite, fullerenes and carbon nanotubes) the $2 s, 2 p_{x}$ and $2 p_{y}$ orbitals hybridize to three $s p^{2}$ hybrid orbitals with an included angle of $120^{\circ}$. These form together with their nearest neighbors covalent $\sigma$-bonds and combine to bonding $\sigma$ and antibonding $\sigma^{*}$ molecular orbitals. The remaining $2 p_{z}$ orbitals, perpendicular to the plane of the $\sigma$ bonds, couple with their neighbors and form delocalized bonding $\pi$ or antibonding $\pi^{*}$ orbitals above and below the plane. They are responsible for the weak interaction between graphene layers in graphite [41], between several shells in MWCNTs or between individual tubes in a bundle of SWCNTs.

The energy levels associated with the $\sigma$-bonds are far away from the Fermi level and therefore they are irrelevant for their electronic properties (fig. 2.5). In contrast, the $\pi$ bands lie in the vicinity of the Fermi energy and therefore are responsible for electronic behavior. In the vertex of the first Brillouin zone (the $\mathrm{K}$ or $\mathrm{K}^{\prime}$ point ${ }^{1}$ ) the $\pi$ bands actually cross the Fermi level (fig. 2.6), which makes graphene a semimetal. Furthermore in the vicinity of the K point the $\pi$ bands are nearly linear.

\subsubsection{Carbon nanotubes: graphene rolled up to a cylinder}

A carbon nanotube results from a graphene sheet rolled up to a cylinder, consequently it exhibits a similar band structure. In the case of nanotubes only one additional constraint has to be fulfilled: going once around the circumference one gets to the same point, i.e. an additional boundary condition for the part of the wave vector $\mathbf{k}$ perpendicular to the tube axis exists:

$$
\Psi_{\mathbf{k}}\left(\mathbf{r}+\mathbf{C}_{\mathbf{h}}\right)=\Psi_{\mathbf{k}}(\mathbf{r})
$$

Due to the Bloch theorem there is a periodic function $u_{\mathbf{k}}(\mathbf{r})=u_{\mathbf{k}}\left(\mathbf{r}+\mathbf{C}_{\mathbf{h}}\right)$ with

$$
\begin{aligned}
\Psi_{\mathbf{k}}(\mathbf{r}) & =e^{i \mathbf{k} \cdot \mathbf{r}} u_{\mathbf{k}}(\mathbf{r}) \\
\Psi_{\mathbf{k}}\left(\mathbf{r}+\mathbf{C}_{\mathbf{h}}\right) & =e^{i \mathbf{k} \cdot \mathbf{r}} e^{i \mathbf{k} \cdot \mathbf{C}_{\mathbf{h}}} u_{\mathbf{k}}\left(\mathbf{r}+\mathbf{C}_{\mathbf{h}}\right)=e^{i \mathbf{k} \cdot \mathbf{r}} e^{i \mathbf{k} \cdot \mathbf{C}_{\mathbf{h}}} u_{\mathbf{k}}(\mathbf{r})
\end{aligned}
$$

\footnotetext{
${ }_{1}$ only two (neighboring) of the six vertices in the first Brillouin zone are different, all others are equivalent to one of these two. Descriptively spoken it can be stated that only a third of every vertex is in the first Brillouin zone and two thirds are outside.
} 

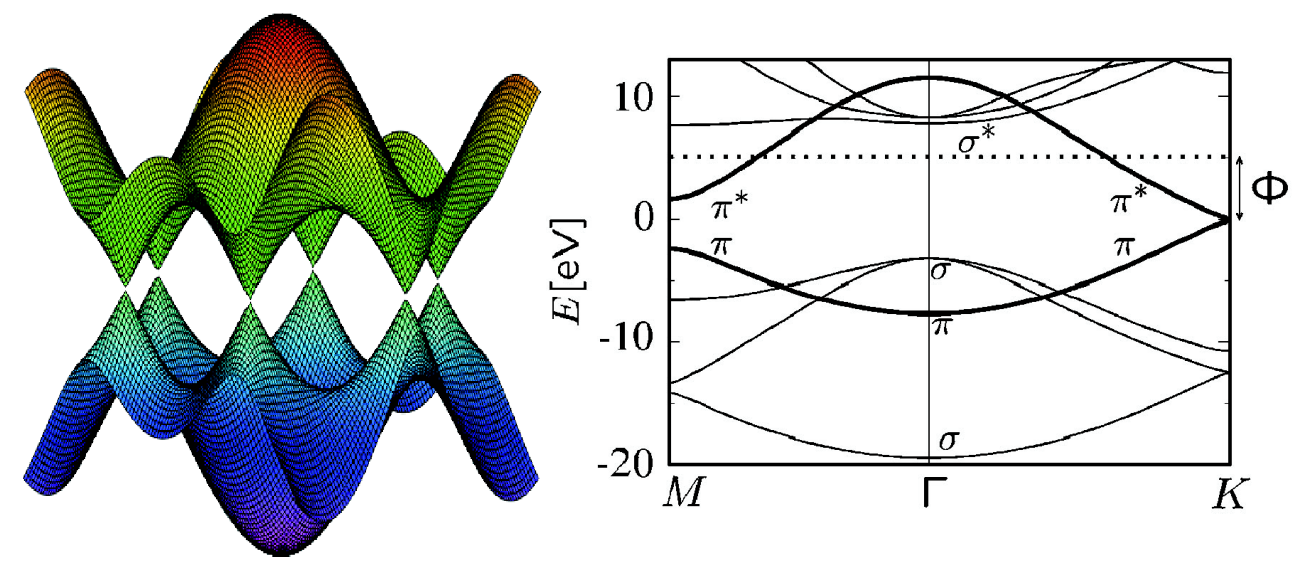

Figure 2.6: Left: the band structure of graphene calculated from the tight-binding model [42] results in the bonding $\pi$ bands (below the Fermi level) and the antibonding $\pi^{*}$ bands (above the Fermi level). The bands touch only at $\mathrm{K}$ and $\mathrm{K}^{\prime}$ the Fermi surface. Right: The bandstructure shows the large band gap between the $\sigma$ and the $\sigma^{*}$ bands. The Fermi energy is set to zero, and $\Phi$ indicates the work function. Adapted from [24]

Comparing equation 2.2 with 2.3 and 2.4 results in the condition $e^{i \mathbf{k} \cdot \mathbf{C}_{\mathbf{h}}}=1$ or

$$
\mathbf{k} \cdot \mathbf{C}_{\mathbf{h}}=2 b \pi \quad \text { with } \quad b \in \mathbb{Z}
$$

This means that only $\mathbf{k}$ vectors are allowed that lie on a line through the $\Gamma$ point in tube direction and parallel in distances of $b \frac{2 \pi}{C_{h}}$. Plotting these allowed lines in the band structure of graphene (fig. 2.6) results in the band structure for the nanotube (see fig. 2.7). If one of the allowed lines hits the $\mathrm{K}$ point, where valence and conduction band are touching each other, the tube is metallic and if all lines miss the $\mathrm{K}$ points there is a band gap: the tube is semiconducting. All armchair nanotubes are metallic because the line through $\Gamma$ hits $K$ and the opposite $K^{\prime}$ $(b=0)$. Generally, the tubes are metallic if $n-m=3 l, l \in \mathbb{Z}$ is fulfilled. All others are semiconducting. As in metallic tubes $K$ and $K^{\prime}$ are intercepted simultaneously $^{1}$, there are two conduction channels. Because the distance of the lines decreases with increasing diameter and the band structure around the $\mathrm{K}$ point is linear, the band gap decreases, too.

The band structure of the carbon nanotubes is one dimensional like the tube itself. It can be derived from that of graphene and the allowed $\mathbf{k}$ vectors by a superposition of the energy dispersions along the lines. The centric line represents

${ }^{1}$ If a line hits the $\mathrm{K}$ point, there is also one hitting $\mathrm{K}^{\prime}(b \rightarrow-b)$ 

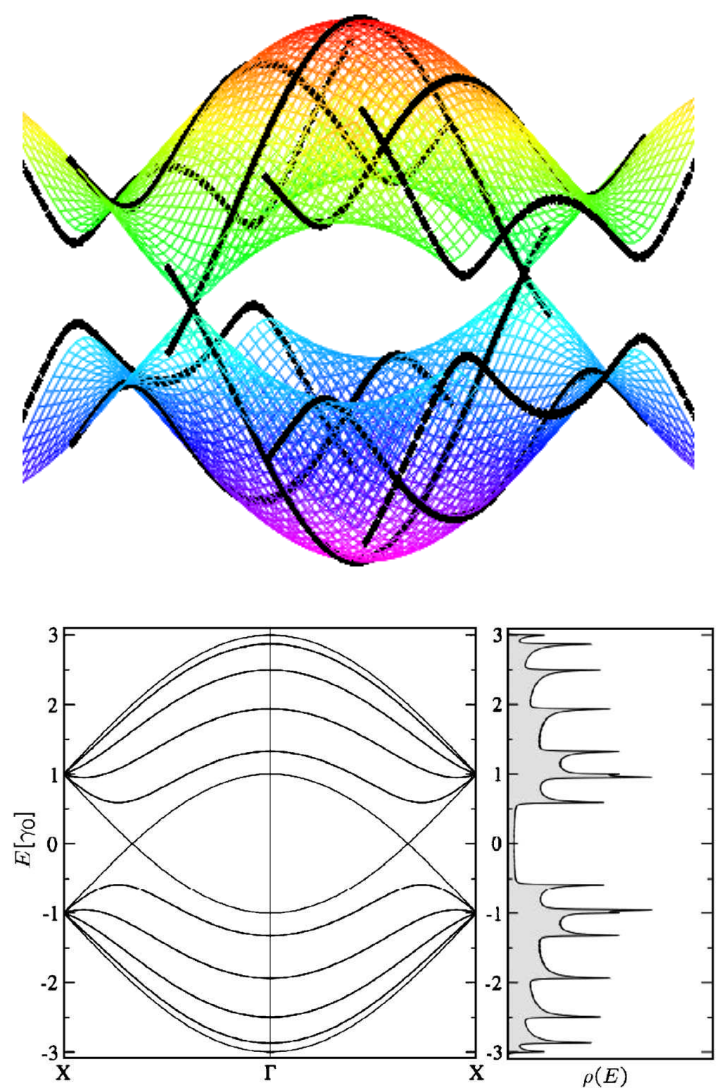

Figure 2.7: Band structure of graphene (calculated from the tight-binding model [42]) together with the energy of the allowed wave vectors (solid lines) for an armchair nanotube. The centric line hits $\mathrm{K}$ and $\mathrm{K}^{\prime}$. The tube is metallic.

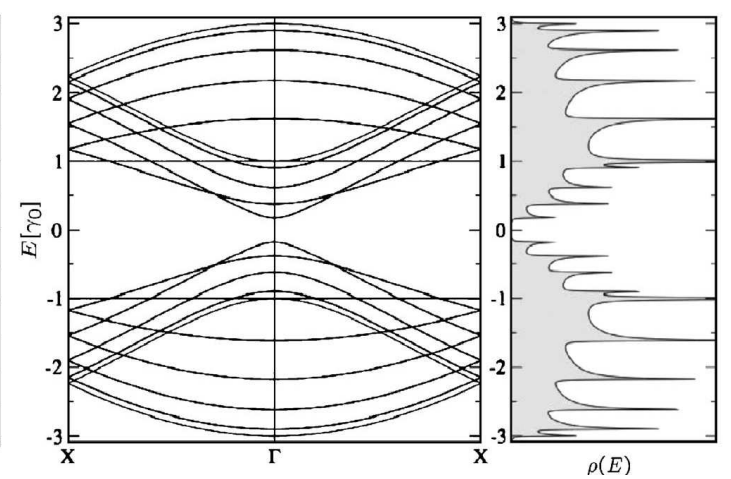

Figure 2.8: Dispersion relation and density of states of a metallic $(5,5)$ armchair nanotube (left) and a semiconducting $(10,0)$ zigzag tube (right). The extrema with horizontal tangents in the band structure result in a diverging density of states (van Hove singularity). $\gamma_{0} \approx 2.9 \mathrm{eV}$. The $\Gamma-X$ direction is axial to the tube. Adapted from [24]

a propagating wave directly in tube direction, the other branches correspond to a helical propagation around the tube. Fig. 2.8 shows the band structure for a metallic $(5,5)$ and a semiconducting $(10,0)$ nanotube.

In a one dimensional system the density of states $\rho(E)=\frac{\mathrm{d} N(E)}{\mathrm{d} E}$ with $\mathrm{d} E=\frac{\partial E}{\partial k} \mathrm{~d} k$ can be written as

$$
\rho(E)=\frac{\mathrm{d} N}{\mathrm{~d} k} \frac{1}{\frac{\partial E}{\partial k}}
$$

Consequently if $\frac{\mathrm{d} E}{\mathrm{~d} k}=0$ (horizontal tangents in the band structure) the density of states diverges (fig. 2.8). This is known as a van Hove singularity [43]. For a metallic nanotube the density of states is constant in the vicinity of the Fermi level. 
All these calculations do not take into account that the graphene sheet is bent into a nanotube. It was predicted, that especially in small diameter tubes with high curvature a small band gap up to about $10 \mathrm{meV}$ appears in all tubes except armchair tubes [44, 45]. This effect was measured by Ouyang et al. [46] in 2001. Even in the highly symmetric armchair nanotubes a small band gap occurs if the tube is in a bundle, due to the intertube interactions [47].

\subsection{Transport properties}

Although it took a rather long time since the discovery of carbon nanotubes 1991 [6] to the first electrical resistance measurement of an individual carbon nanotube 1996 [48], some transport properties like magnetoresistance were already known from measurements on bundles of nanotubes $[49,50]$.

\subsubsection{Quantum conductance}

In a macroscopic material the resistivity is a material constant and independent of the geometry. In mesoscopic systems for dimensions in the range of the mean free path $L_{m}$ or of the phase coherence length $L_{\varphi}$, however, quantum conductance effects appear. If the length of the contacted nanotube $L$ becomes smaller than both, the mean free path and the phase coherence length, the intrinsic resistance is independent of $L$. In this regime of ballistic transport there is not one Fermi energy $E_{F}$ defined, but two chemical potentials $\mu_{l}$ for the $\mathbf{k}$ vectors from left to right and $\mu_{r}$ for the oposite direction, equal to the Fermi energy of the left and right electrode, respectively. The conductance is $G=M \frac{2 e^{2}}{h}=M G_{0}$ where $M$ is the number of bands with electronic states between $\mu_{l}$ and $\mu_{r}$ [51]. In carbon nanotubes with the Fermi energy at the charge neutrality point ${ }^{1}$ and low bias the number of channels is $M=2$, according to the touching valence and conductance bands on $\mathrm{K}$ and $\mathrm{K}^{\prime}$. In the clean limit with no scattering in the tube only the electrodes can disturb the wave propagation because of non ideal contacts (tunneling) or backscattering. For disordered or multiwalled tubes $L_{m}$ as a function of the Fermi level was calculated [52, 53] and measured [54].

Several groups reported ballistic transport in MWCNTs for tube lengths up to several $\mu \mathrm{m}$ even at room temperature [55-59]. Surprisingly the early experiments of Frank et al. [55] revealed a quantum conductance of only $G=1 \mathrm{G}_{0}$. Subsequent

\footnotetext{
${ }^{1}$ the energy level where $\pi$ and $\pi^{*}$ crosses
} 
experiments measured the predicted value of $G=2 \mathrm{G}_{0}[56]$ but also a conductance up to $G=490 \mathrm{G}_{0}[57]$. In all these experiments the nanotubes were grown directly on one of the electrodes. In single-wall tubes also ballistic transport was reported even between evaporated electrodes, measured with an electric force microscope (EFM) [60].

In the more common contacting method with evaporated contacts, diffusive transport was reported for MWCNTs [48, 54, 60-65], but showing several quantum conductance effects.

Conductance measurements in a magnetic field can identify and distinguish weak localization, universal quantum fluctuations and Altshuler-Aronov-Spivac oscillations. If $L_{m}<<L_{\varphi}$ there are many scattering centers conserving phase coherence. Closed paths containing only phase coherent scatterers can be passed in both directions. Since the path and the phase of both parts of the wave is equal they interfere constructively. The increased probability of presence after passing the closed path is equivalent to an increased probability of backscattering. The electron mobility is decreased and therefore also the conductivity decreases. This is known as weak localization. At low temperatures the phase destroying electronphonon and electron-electron scattering events are reduced and the resistance is increased due to weak localization.

Applying a magnetic field perpendicular to the closed path adds (subtracts) a phase difference to the two parts of the wave and destroys the constructive interference. A magnetoresistance peak at zero field indicates a weak localization regime. Depending on the tube and the distribution of the scattering centers there are additional smaller peaks in the magnetoresistance known as universal conductance fluctuations. They originate from interference effects between different paths. A magnetic field changes the phase relations between different paths and leads to randomly distributed but reproducible oscillations. Weak localization and universal conductance fluctuations were observed e.g. in refs. [54, 65, 66].

If the magnetic field is applied parallel to the tube only phases of paths going at least once around the tube are altered. Since the phase difference depends linearly on the enclosed flux and all paths once around the tube include the same flux, there are periodic positions in the magnetic field where interference is constructive and therefore the conductance is reduced [67]. In 1999 these oscillations were observed in multiwall tubes, since the required magnetic fields are only achievable for large diameter tubes [68]. Additionally this experiment showed that only the outer shells with large diameter contribute to the current at low temperature. 
In the case of weakly coupled electrodes i.e. high contact resistance, Coulomb blockade dominates electronic transport [69, 70]. This effect originates from the charging energy when one electron is added to the tube and suppresses conductance if no energy level lies between the voltages on the electrodes. It was also reported that prestructured electrodes divide one single nanotube in several quantum dots due to bending near the edges of electrodes [71].

Other quantum conductance effects like negative four point resistance [72] that originate from backscattering effects at impurities and subsequent interference of multiply reflected waves, or Luttinger-liquid behavior [73] have been reported.

\subsubsection{Conductance at room temperature}

The described quantum conductance effects occur (except ballistic transport with tubes grown on the electrodes) only at low temperatures. For higher temperatures up to room temperature the results for multiwall and single-wall nanotubes differ strongly. With EFM measurements Bachtold et al. [60] found clear evidence for diffusive transport in a MWCNT and with the same setup no intrinsic resistance in metallic SWCNTs. For semiconducting SWCNTs, however, defects in the tubes dominated electron transport.

An interesting experimental setup was used by Yaish et al. [62]. The authors have injected current in a SWCNT using evaporated Au contacts and used the conducting tip of an atomic force microscope (AFM) as a local voltage probe. In this way the contact resistances could easily be measured directly to be $15 \mathrm{k} \Omega$ independent of the gate voltage. However, the potential drop near the electrodes depended strongly on the gate voltage. Their results idicated that the Au contacts induce Schottky barriers in n-type semiconducting tubes as observed in previous experiments $[74,75]$. On the other hand the potential drop away from the contact electrodes was linear indicating diffusive transport for distances of at least $200 \mathrm{~nm}$.

Nonlinear resistance versus length was found by Pablo et al. [61] also with a conductive AFM but only in a two point setup with one evaporated electrode and the other provided by the AFM tip. The authors conclude that this is due to the presence of nondissipative scattering centers and that electron transport is coherent even for tubes with high intrinsic resistances. But it is also necessary to think about possible Schottky barriers at the electrodes that result in an unexpected scaling behavior [74].

An experiment similar to that of Yaish et al. [62] but with even less invasive voltage electrodes was carried out by Gao et al. [72]. They used two MWCNTs as 
voltage probes in a four point setup and moved them with an AFM tip in order to measure the four point resistances for different lengths. At room temperature they found the resistance to be linear with distance, indicating a diffusive incoherent limit. Additionally the four point resistance remains constant at temperatures above $80 \mathrm{~K}$, suggesting that the intrinsic resistance is due to disorder and not to phonon scattering. Other groups found contrarily a domination of electron-phonon scattering at room temperature [65, 76-78].

\subsubsection{The role of defects and multiple shells}

The discrepancy in the results described above are on the one hand due to differences in tube diameter and chirality but on the other hand definitely due to differences in the quality of the nanotube material. An often practiced method to visualize defects is scanning gate microscopy (SGM). Here a charged AFM tip is scanned over the sample at constant height as a local gate and the the two point resistance is recorded as a function of the the tip position. Since defects in nanotubes have a large impact on the conductance a local change in Fermi energy at a defect alters the conductance much more than at other positions. It was found, that especially in semiconducting tubes voltage drop occurs mainly at defects $[60,79]$. Since in MWCNTs the shells are typically incommensurate they introduce an aperiodic potential that can be handled as a defect density [80], the SGM detects defects all over the tube. Therefore this method is not as effective as in SWCNTs.

From a theoretical point of view defects can be handled with a disorder parameter that is constant over the whole tube as it was done by Triozon et al. [52]. The result was a diffusive transport with $L_{m}$ scaling with the tube diameter and being strongly dependent on the Fermi level. At least the latter has been confirmed experimentally by Stojetz et al. [54].

Alternatively, individual defects can be modeled and their impact on the density of states or transmission probability can be calculated. This was performed for example by Rochefort et al. [81] with bent nanotubes and a strong decrease in transmission probability due to $\sigma-\pi$ hybridization effects was found. Other groups reported a decrease of the density of states in bent nanotubes [82]. Strain also reduces the density of states [83].

A lot of experimental work was done in characterizing the electronic behavior of carbon nanotubes with defects. Bending multiwall nanotubes, measuring their resistance and simultaneously recording TEM images of the defects revealed that 
conductance decreases with curvature and stronger bendings result in plastic deformation of the tube [84]. Other groups reported increasing resistance with increasing strain, in some works combined with bending of the tube [85-87]. In the present work these results were confirmed and the resistance increase could be assigned directly to modifications in the tube structure.

Additionally to the defect density the incommensurability of the multiple shells hinders a complete theoretical description of a MWCNT. It is often included in the models as an aperiodic perturbation potential. This disturbance alone changes the transport in a diffusive regime in every shell but with a long mean free path $[53,80]$. The next question is how much of these shells contribute to the total conductance. Several groups provide indirect arguments that the current flows at least at low temperature predominantly in the outermost shell $[54,55,68]$. Calculations, however, are discordant. The predictions depend much more on the model used than on the tube parameters [52, 88-92]. They range from suppressed intershell transport for a long tube [90], to the result that the wave function spreads over several shells [89].

There are also experiments indicating a not negligible intershell conductance. One example are electrical breakdown experiments, where only the outermost shell is contacted and then parts of the shells in between the electrodes are removed step by step [93]. At every removal step a part of the conductance is lost according to the removed shell. Nevertheless current can flow up to ten removal steps indicating a large intershell conductance. Further experiments, where the current is forced into lower shells, are a tube with an incomplete outermost shell [64] or telescopically extended tubes [37]. Calculations on the latter setup result in at least nonzero transmission and show a conductance which scales linearly with the length of the overlap region $[36,91]$.

Tubes without broken shells were used in only one study for deducing the intershell conductance [94]. Here an array of electrodes were evaporated equidistantly on a single tube and were used for current injection and voltage measurement, in a local or a nonlocal geometry. The analysis with a simple resistivity model that neglected the influence of the finite electrode dimensions revealed for a $1 \mu \mathrm{m}$ long tube an intershell conductance and an intrashell resistance in the same range of magnitude. 



\section{The resistance network model of a MWCNT}

It is not simple to calculate theoretically how much current flows in each shell of a multiwall carbon nanotube. Predictions evaluated from several models assuming quantum transport differ widely as described in the previous chapter. For room temperature, however, diffusive transport was also reported $[48,54,60-65]$. If inelastic scattering is dominant, $L_{m}$ and $L_{\varphi}$ are in the same range and for tubes much longer than these lengths, classical transport can be assumed.

The classical resistor model suggested by Bourlon et al. [94] is presented in section 3.1. It assumes an infinitely long tube, infinitely small electrodes and uses three free parameters: the intrashell resistivity ${ }^{1} \rho_{o}$ and $\rho_{b}$ for the outermost and the shell below, respectively, and the intershell conductivity ${ }^{2}$. Intrashell resistance and intershell conductance were used because both scale linearly with length.

Section 3.2 describes an improved model developed within this thesis taking into account the finite dimensions of the electrodes and of the tube.

\subsection{Punctual current injection in an infinitely long tube}

An infinitely long double wall nanotube contacted with two contact electrodes of separation $L$ that connect only to the outer shell is considered to be like a resistor model shown in fig. 3.1. The shells are considered to be one dimensional and they are modeled with a resistor array. The intershell conductance is represented by resistors between the two shells. For infinitely small resistor cells, the current distribution can be handled with a system of differential equations which can be solved analytically.

\footnotetext{
${ }^{1}$ resistance per length in this $1 \mathrm{D}$ case

${ }^{2}$ conductance per length
} 

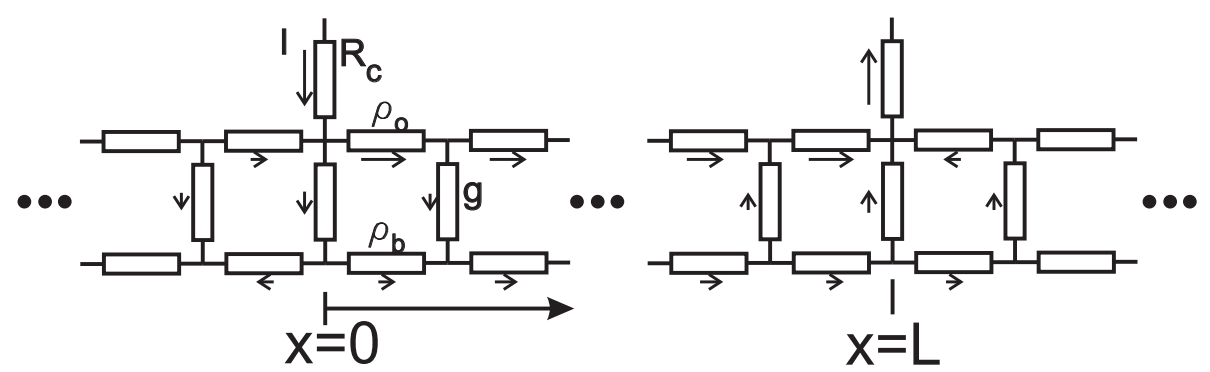

Figure 3.1: The resistor network of a double-wall nanotube considering classical transport through and between the shells. The current $I$ enters the tube through the contact resistance, flows through the network (illustrated with arrows) and leaves the tube again through the second contact resistor at $x=L$.

The relation between voltage $V(x)$ and current $I(x)$ within the outermost shell (index $o$ ) or the shell below (index $b$ ) is:

$$
\begin{aligned}
& \frac{\mathrm{d} V_{o}(x)}{\mathrm{d} x}=-\rho_{o} I_{o}(x) \\
& \frac{\mathrm{d} V_{b}(x)}{\mathrm{d} x}=-\rho_{b} I_{b}(x)
\end{aligned}
$$

The variation of the current in the shell depends on the voltage difference between the two shells and the intershell conductance $g$ :

$$
\begin{aligned}
& \frac{\mathrm{d} I_{o}(x)}{\mathrm{d} x}=g\left(V_{b}(x)-V_{o}(x)\right) \\
& \frac{\mathrm{d} I_{b}(x)}{\mathrm{d} x}=g\left(V_{o}(x)-V_{b}(x)\right)
\end{aligned}
$$

Differentiating equation 3.3 again and using equations 3.1 and 3.2 results in:

$$
\frac{\mathrm{d}^{2} I_{o}(x)}{\mathrm{d} x^{2}}=-g\left(\rho_{b} I_{b}(x)-\rho_{o} I_{o}(x)\right)
$$

\subsubsection{Beyond the electrodes}

Since in the zone beyond the electrodes there is no total current and therefore $I_{o}(x)=-I_{b}(x)$, equation 3.5 can be rewritten as:

$$
\frac{\mathrm{d}^{2} I_{o}(x)}{\mathrm{d} x^{2}}=g I_{o}(x)\left(\rho_{o}+\rho_{b}\right)
$$


with the solution:

$$
\begin{aligned}
I_{o}(x) & =J_{1} \exp \left(\frac{x}{L_{a}}\right)+J_{2} \exp \left(-\frac{x}{L_{a}}\right) \\
\text { with } L_{a} & =\frac{1}{\sqrt{g\left(\rho_{o}+\rho_{b}\right)}}
\end{aligned}
$$

For symmetry reasons the parts of the tube beyond the injection electrodes are equivalent and consequently it is sufficient to calculate the left part $(x<0)$. Since the current for $x \rightarrow-\infty$ should be zero or at least not diverge, $J_{2}$ has to be set to zero. $I_{o}\left(0^{-}\right)=J_{1}$ i.e. $J:=J_{1}$ can be interpreted as the current that leaves the region between the electrodes in the inner shell and flows back to the contact in the outermost shell. The result for current and voltage left beyond the electrodes is:

$$
\begin{aligned}
I_{o}(x) & =J \exp \left(\frac{x}{L_{a}}\right) \\
V_{o}(x) & =-\rho_{o} \int_{0}^{x} I_{o}(t) \mathrm{d} t=\rho_{o} L_{a} J\left(1-\exp \left(\frac{x}{L_{a}}\right)\right)
\end{aligned}
$$

\subsubsection{Between the electrodes}

In between the current injecting electrodes the total current is $I$ i.e. $I_{b}(x)=$ $I-I_{o}(x)$. Together with equation 3.5 this results in:

$$
\frac{\mathrm{d}^{2} I_{o}(x)}{\mathrm{d} x^{2}}=g I_{o}(x)\left(\rho_{o}+\rho_{b}\right)-g \rho_{b} I
$$

The general solution is:

$$
I_{o}(x)=c_{1} \exp \left(-\frac{x}{L_{a}}\right)+c_{2} \exp \left(\frac{x}{L_{a}}\right)+\frac{\rho_{b} I}{\rho_{o}+\rho_{b}}
$$

The total current entering at the electrode splits in currents $I_{o}\left(0^{-}\right)$and $I_{o}\left(0^{+}\right)$:

$$
I_{o}\left(0^{+}\right)=I+J
$$

and because of symmetry:

$$
I_{o}\left(0^{+}\right)=I_{o}\left(L^{-}\right)
$$

whereby $L$ denotes the distance between the current electrodes. Another boundary condition results from the continuity and differentiability of $I_{b}$ : $I_{b}\left(0^{-}\right)=I_{b}\left(0^{+}\right)$ 


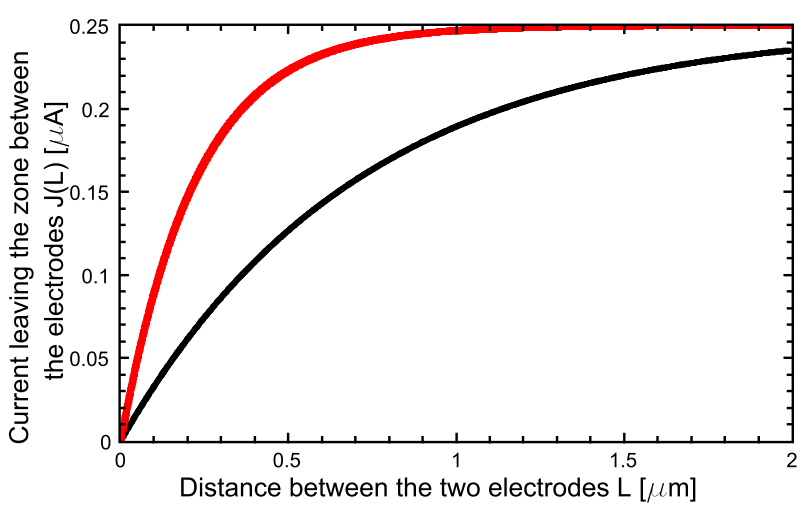

Figure 3.2: The current J leaving the zone between the electrodes for two sets of parameters: $I=$ $1 \mu \mathrm{A}, \rho_{o}=\rho_{b}=10000 \Omega / \mu \mathrm{m}$; $g=10000^{-1} \Omega^{-1} / \mu \mathrm{m}$ (black); $g=1000^{-1} \Omega^{-1} / \mu \mathrm{m}$ (red). Both curves saturate at $0.25 \mu \mathrm{A}$ independently of $g$.

and $I_{u}^{\prime}\left(0^{-}\right)=I_{u}^{\prime}\left(0^{+}\right)$(result of equation 3.4 which is valid for the complete tube). Since there is

$$
\begin{array}{lll}
x<0: I_{o}(x)=-I_{b}(x) & \Longrightarrow & \frac{\mathrm{d} I_{o}(x)}{\mathrm{d} x}=-\frac{\mathrm{d} I_{b}(x)}{\mathrm{d} x} \\
x>0: I_{o}(x)=I-I_{b}(x) & \Longrightarrow & \frac{\mathrm{d} I_{o}(x)}{\mathrm{d} x}=-\frac{\mathrm{d} I_{b}(x)}{\mathrm{d} x}
\end{array}
$$

it also holds:

$$
I_{o}^{\prime}\left(0^{+}\right)=I_{o}^{\prime}\left(0^{-}\right)
$$

Together with equations 3.12 and 3.9 this is equivalent to:

$$
-\frac{c_{1}}{L_{a}}+\frac{c_{2}}{L_{a}}=\frac{J}{L_{a}}
$$

Solving the equation system of $3.13,3.14$ and 3.18 results in:

$$
\begin{aligned}
I_{o}(x) & =\frac{\rho_{o} I}{2\left(\rho_{o}+\rho_{b}\right)}\left(\exp \left(-\frac{x+L}{L_{a}}\right)+\exp \left(\frac{x}{L_{a}}\right)\right)+\frac{\rho_{b} I}{\rho_{o}+\rho_{b}} \\
J(L) & =-\frac{\rho_{o} I}{2\left(\rho_{o}+\rho_{b}\right)}\left(1-\exp \left(-\frac{L}{L_{a}}\right)\right) \\
V_{o}(x) & =-\rho_{o} \int_{0}^{x} I_{o}(t) \mathrm{d} t= \\
& =\frac{\rho_{o} I}{2\left(\rho_{o}+\rho_{u}\right)}\left(\rho_{o} L_{a}\left(1-\exp \left(-\frac{1}{L_{a}}\right)-\exp \left(\frac{1}{L_{a}}\right)+\exp \left(-\frac{x+1}{L_{a}}\right)\right)-2 \rho_{b} x\right)
\end{aligned}
$$

\subsubsection{Discussion}

In equation $3.20 I_{b}(0)=-J(L)$ is the current exceeding the zone between the two electrodes. This is plotted in fig. 3.2 for two sets of parameters as a function of 

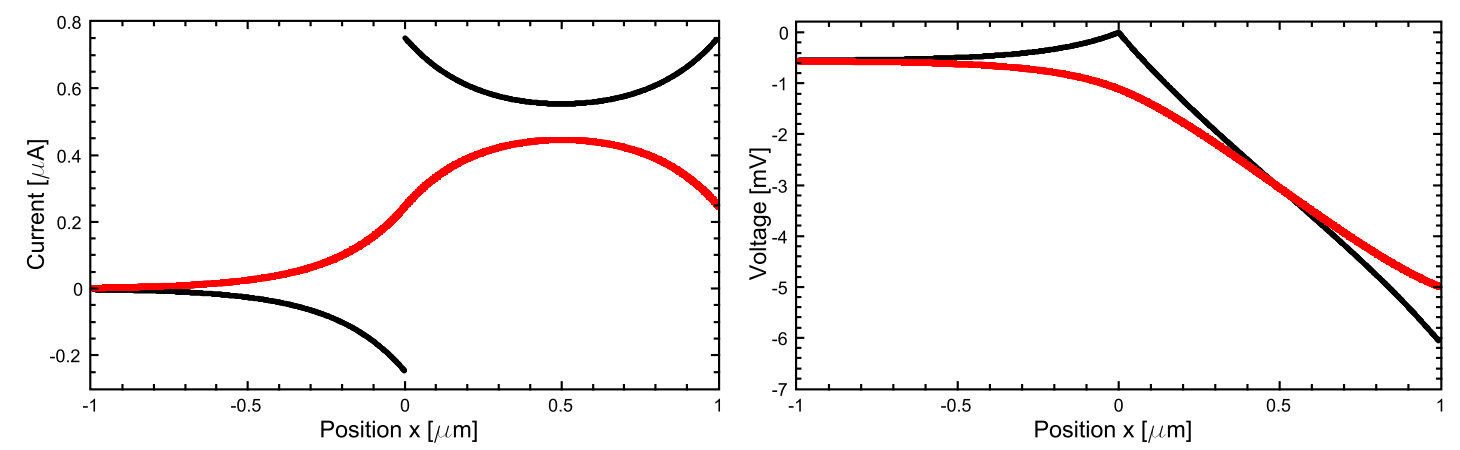

Figure 3.3: Current (left) and voltage (right) in the outermost (black) and in the inner shell (red) for a electrode separation $L=1 \mu \mathrm{m} . \quad I=1 \mu \mathrm{A}, \rho_{o}=\rho_{b}=$ $10000 \Omega / \mu \mathrm{m}, g=1000^{-1} \Omega^{-1} / \mu \mathrm{m}$

the distance between the contacts $L$. For large $L$ it saturates at $J_{\max }=\frac{\rho_{o} I}{2\left(\rho_{o}+\rho_{b}\right)}$. Since $V_{o}(x<0)$ (eq. 3.10) depends linearly on $J$, the nonlocal voltage depends also strongly on $L$, especially for $L<L_{a}$.

Fig. 3.3 shows the current and the voltage in the outermost and the inner shell of the tube. The jump in $I_{o}$ at $x=0$ is a result of the punctual current injection at this point. With the parameters in fig. 3.3 the decay in the nonlocal current and voltage is nearly completed at $x=-1$. Note that not only $U_{b}(x)$ is bent, but a slight bending can also be observed in $U_{o}(x)$, the only measurable effect of the intershell conductance that can be evidenced in the local voltage.

\subsection{Continuous current injection and finite tube length}

In the model of Bourlon et al. [94] described above, the current is injected from two point contacts. In their experiment, however, current was injected and voltage was measured via $200 \mathrm{~nm}$ broad evaporated electrodes separated by $200 \mathrm{~nm}$ in between. Due to experimental limitations, the injection zone, however, cannot be further downsized. Therefore the jump at $I_{o}(x=0)$ is clearly unrealistic. To create a model closer to the reality we replaced the punctual injection by an expanded injection zone below the evaporated electrode (illustration in fig. 3.4) with length $l_{p}$. Another conflict of Bourlon's model with reality is the infinite length of the tube. Therefore an additional parameter $l_{l}$ is included, representing the length of the protruding part of the tube. 

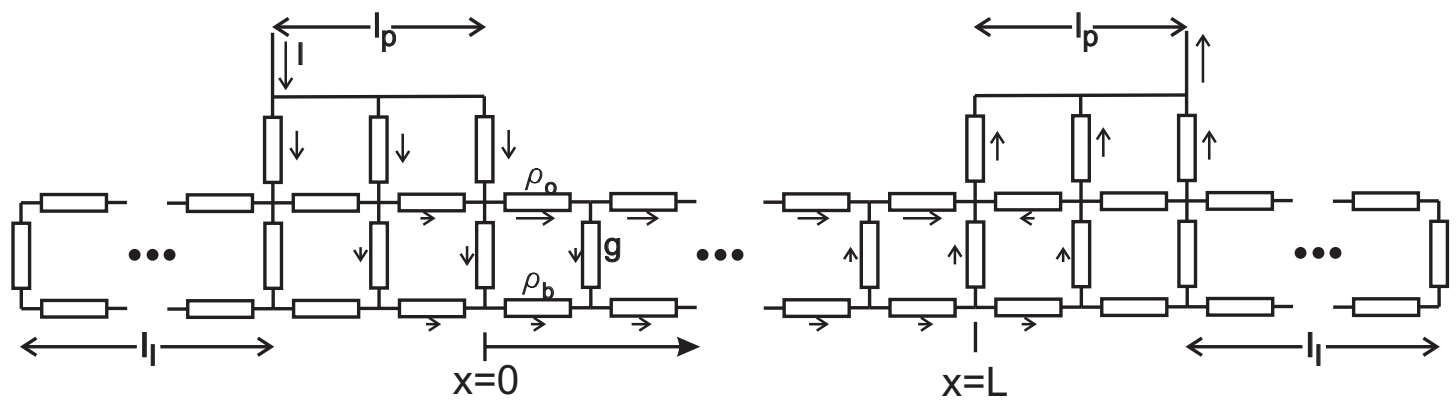

Figure 3.4: The resistor model for continuous current injection. The current can enter the outermost shell in the whole injection zone below the electrode.

The differential equations for $x<-l_{p}$ and $0<x<L$ are the same as in section 3.1:

$$
\begin{aligned}
x<-l_{p}: & \frac{\mathrm{d}^{2} I_{o}(x)}{\mathrm{d} x^{2}}=g I_{o}(x)\left(\rho_{o}+\rho_{b}\right) \\
0<x<L: & \frac{\mathrm{d}^{2} I_{o}(x)}{\mathrm{d} x^{2}}=g I_{o}(x)\left(\rho_{o}+\rho_{b}\right)-g \rho_{b} I
\end{aligned}
$$

but the boundary conditions differ from those of Bourlon's model.

\subsubsection{Injection zone}

The metallic electrode is assumed to be a good conductor with resistivity $\rho_{p} \cong 0$. Consequently the derivative of the voltages in the three layers pad $\left(V_{p}\right)$, outermost shell $\left(V_{o}\right)$ and inner shell $\left(V_{b}\right)$ are:

$$
\begin{aligned}
& \frac{\mathrm{d} V_{p}(x)}{\mathrm{d} x}=0 \\
& \frac{\mathrm{d} V_{o}(x)}{\mathrm{d} x}=-\rho_{o} I_{o}(x) \\
& \frac{\mathrm{d} V_{b}(x)}{\mathrm{d} x}=-\rho_{b} I_{b}(x)
\end{aligned}
$$

For the whole tube (injection zone included) it holds:

$$
\frac{\mathrm{d} I_{b}(x)}{\mathrm{d} x}=g\left(U_{o}(x)-U_{b}(x)\right)
$$

Below laterally extended contact electrodes, the variation of $I_{o}(x)$ depends not only on $g$ and the difference between $U_{o}(x)$ and $U_{b}(x)$ but also on the contact 
conductivity $\sigma$ and the difference between $U_{o}(x)$ and the constant $U_{p}(x)=U_{p}$ :

$$
\frac{\mathrm{d} I_{o}(x)}{\mathrm{d} x}=g\left(U_{b}(x)-U_{o}(x)\right)+\sigma\left(U_{p}-U_{o}(x)\right)
$$

Once again, differentiating equation 3.28 and inserting 3.24 - 3.26 results in:

$$
\frac{\mathrm{d}^{2} I_{o}(x)}{\mathrm{d} x^{2}}=g\left(\rho_{o} I_{o}(x)-\rho_{b} I_{b}(x)\right)+\sigma \rho_{o} I_{o}(x)
$$

Since the electrode is a conductor with $\rho_{p} \cong 0$ the injection point in the electrode is not relevant. Nevertheless for this model it is useful to consider the connection points to be the leftmost (rightmost) point of the left (right) electrode. In this case, the total current for all three layers is $I$, i.e. $I_{b}(x)=I-I_{p}(x)-I_{o}(x)$. Inserting in equation 3.29 results in:

$$
\frac{\mathrm{d}^{2} I_{o}(x)}{\mathrm{d} x^{2}}=g\left(\rho_{o} I_{o}(x)-\rho_{u}\left(I-I_{o}(x)-I_{p}(x)\right)\right)+\sigma \rho_{o} I_{o}(x)
$$

The current leaving the electrode is equal to the injected current in the outermost shell:

$$
\left.\frac{\mathrm{d} I_{p}(x)}{\mathrm{d} x}=\sigma\left(U_{o}(x)-U_{p}\right)\right)
$$

Differentiating this equation and inserting equations 3.24 and 3.25 results in the last required differential equation:

$$
\frac{\mathrm{d}^{2} I_{p}(x)}{\mathrm{d} x^{2}}=-\sigma \rho_{o} I_{o}(x)
$$

\subsubsection{Boundary conditions}

Although all described differential equations are analytically solvable and the boundary conditions can be fixed accordingly, it was not possible to generate a solution for asymmetrically contacted tubes due to limitations of computer power ${ }^{1}$. For symmetrically contacted tubes the boundary condition for the end of the tube is:

$$
I_{o}\left(-l_{l}-l_{p}\right)=0
$$

with the length of the protruding part of the tube on one side $l_{l}$ and the width of the electrode $l_{p}$. Since everywhere only an infinitesimal amount of current is

\footnotetext{
${ }^{1}$ The solution for symmetrically contacted tubes will not be displayed explicitly because it fills several tenths of pages.
} 
injected, $I_{o}(x)$ has to be continuous:

$$
\begin{gathered}
I_{o}\left(-l_{p}^{-}\right)=I_{o}\left(-l_{p}^{+}\right) \\
I_{o}\left(0^{-}\right)=I_{o}\left(0^{+}\right)
\end{gathered}
$$

and for symmetry reasons:

$$
I_{o}(0)=I_{o}(L)
$$

The current in the electrode starts at $x=-l_{p}$ with $I$ and decreases to 0

$$
\begin{aligned}
I_{p}\left(-l_{p}\right) & =I \\
I_{p}(0) & =0
\end{aligned}
$$

The last two required boundary conditions result from the differentiability of $I_{b}(x)$ (see eq. 3.27):

$$
\begin{array}{cc}
x<-l_{p}: I_{o}(x)=-I_{b}(x) & \Rightarrow \frac{\mathrm{d} I_{b}(x)}{\mathrm{d} x}=-\frac{\mathrm{d} I_{o}(x)}{\mathrm{d} x} \\
-l_{p}<x<0: I=I_{p}(x)+I_{o}(x)+I_{b}(x) & \Rightarrow \frac{\mathrm{d} I_{b}(x)}{\mathrm{d} x}=-\frac{\mathrm{d} I_{o}(x)}{\mathrm{d} x}-\frac{\mathrm{d} I_{p}(x)}{\mathrm{d} x} \\
\Longrightarrow \quad I_{o}^{\prime}\left(-l_{p}^{-}\right)=I_{o}^{\prime}\left(-l_{p}^{+}\right)+I_{p}^{\prime}\left(-l_{p}^{+}\right)
\end{array}
$$

Similarly for $x=0$ :

$$
I_{o}^{\prime}\left(0^{-}\right)+I_{p}^{\prime}\left(0^{-}\right)=I_{o}^{\prime}\left(0^{+}\right)
$$

The system of differential equations given by 3.22, 3.23, 3.30 and 3.32 together with the boundary conditions 3.33 - 3.38 and 3.41 - 3.42 was solved using Maple.

Unlike in the model with punctual current injection, the contact resistance with the voltage step between $U_{p}\left(0^{-}\right)$and $U_{o}\left(0^{+}\right)$is not obvious, but has to be calculated from equation 3.31. If the electrode is set to ground potential $U_{p}=0$, the voltage at the tube resulting from the contact resistance at $x=0^{-}$is:

$$
U_{o}\left(0^{-}\right)=\frac{1}{\sigma}\left(I_{p}^{\prime}\left(0^{-}\right)+U_{p}\right)=\frac{I_{p}^{\prime}\left(0^{-}\right)}{\sigma}
$$

Consequently the voltage for the outermost shell of the tube is:

$$
U_{o}(x)=\frac{I_{p}^{\prime}\left(0^{-}\right)}{\sigma}-\rho_{o} \int_{0}^{x} I_{o}(t) \mathrm{d} t
$$




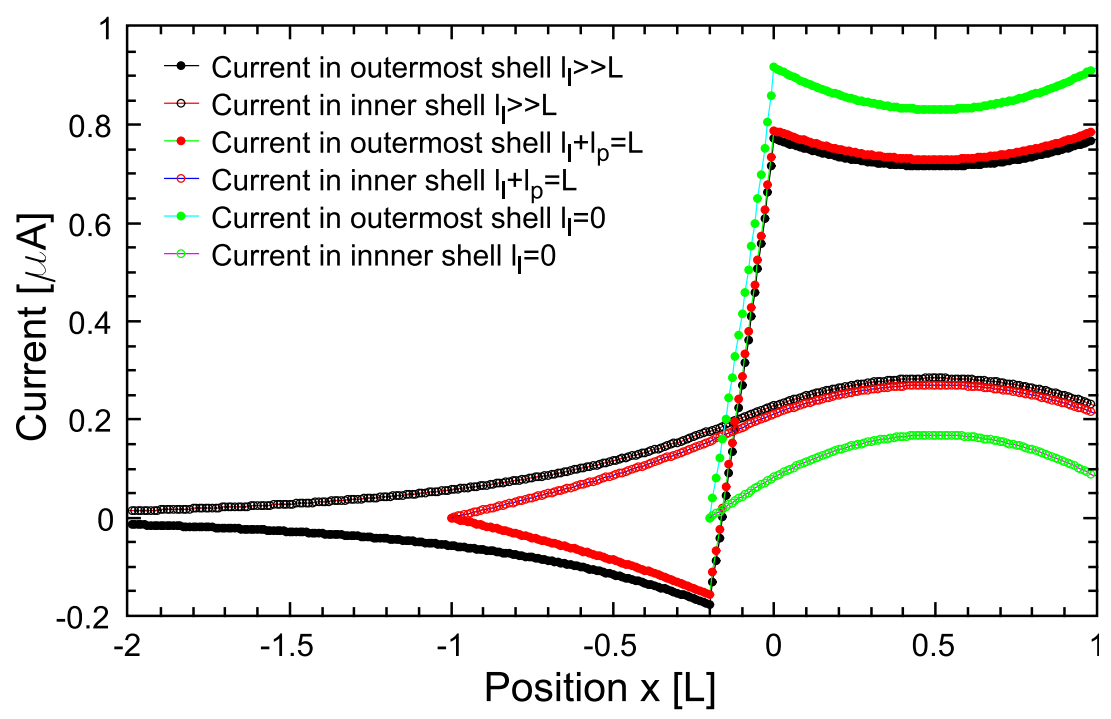

Figure 3.5: Current in the outermost and the inner shell calculated with continuous current injection for varying lengths. $I=1 \mu \mathrm{A}, \rho_{o}=\rho_{b}=10 \mathrm{k} \Omega / \mathrm{L}$, $g=10^{-1} \mathrm{k}^{-1} \Omega / \mathrm{L}, \sigma=0.002 \Omega^{-1} / \mathrm{L}$ and $l_{p}=0.2 L$.

And similarly:

$$
U_{b}(x)=-\frac{I_{b}^{\prime}\left(0^{-}\right)}{g}+\frac{I_{p}^{\prime}\left(0^{-}\right)}{\sigma}-\rho_{b} \int_{0}^{x} I_{b}(t) \mathrm{d} t
$$

\subsubsection{Discussion}

Since the differential equations for the region between and the region beyond the electrodes are the same as in the model with punctual current injection the solutions appear similar. Especially in the region between the electrodes, the numerical values of the constants differ only slightly.

Due to the finite length of the tube, in the region beyond the electrodes no constant can be set to zero. This is a result of the current decay having to be complete at $x=-l_{l}-l_{p}$. A result is that the nonlocal current as well as the maximum current in the inner shell depend strongly on $l_{l}$ (see fig. 3.5). Because the local potential is proportional to the integral of the current $I_{o}(x)$, both the gradient and the bending of the potential profile between the electrodes depend on the total length (see fig. 3.7). Furthermore, the bending depends mainly on the ratio of $\rho_{u}$ to $\rho_{o}$ and on $g$. For small intershell conductance evidently only a small amount of current can enter the inner shell. It is equally clear that the current prefers to flow in the inner shell if $\rho_{b}<\rho_{o}$. 


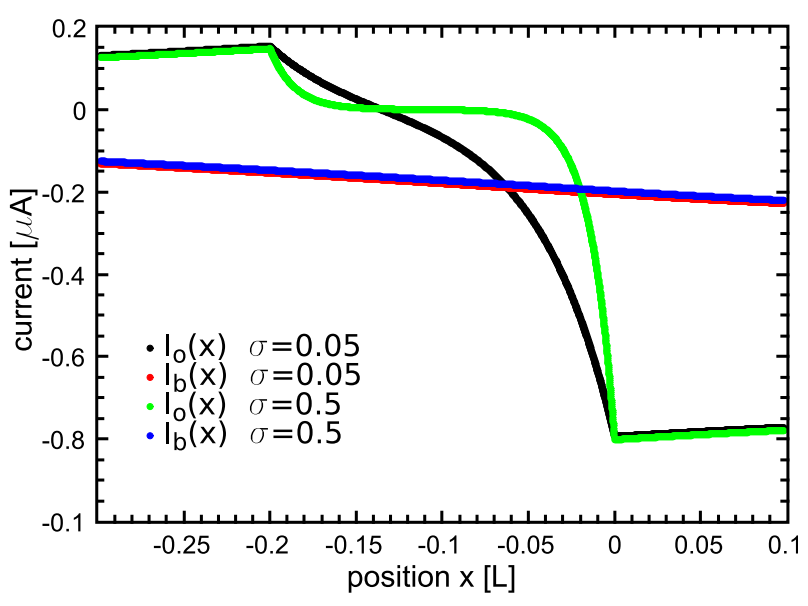

Figure 3.6: The current distribution in the injection region strongly depends on $\sigma$. For small $\sigma$ the current decays linearly in the outer shell, while for larger $\sigma$ or larger electrodes injection occurs mostly at the edge of the electrodes. The current in the inner shell is only slightly dependent on $\sigma$. Except $\sigma$, the parameters are the same as in fig. 3.5 .

The current in the injection region depends strongly on the contact conductivity $\sigma$. For a small conductance the current density is nearly homogeneous in the whole injection region leading to a linear increase of current in the outermost shell. A higher value for $\sigma$ leads to an injection mainly at the ends of the electrodes and consequently reduces the current below the center (fig. 3.6). Large electrodes amplify this effect. Therefore the contradiction between the results of Wakaya et al. [95] that the contact resistance depends on the contact length and that of Mann et al. [96] that the current injection mainly occurs on the electrode edges, can be traced back to differences in the contact conductivity. This is plausible since Mann et al. used mainly Pd contacts known to have considerably lower contact resistivity than the $\mathrm{Ti} / \mathrm{Au}$ contacts used by Wakaya et al..

Since the current density between the electrode and the outermost shell $\frac{\mathrm{d} I_{p}(x)}{\mathrm{d} x}=$ $\sigma U_{o}(x)$ are small in the center of the contact, the voltage is also small. Nevertheless the contact resistance defined by the voltage $I_{o}$ at $x=0$ is finite because of the finite integral over the region where the current is actually injected.

Fig. 3.7 shows the potential profile for the outermost shell for different tube lengths. The local potential between the electrodes remains nearly constant for $l_{l} \geq L$ but changes noticeably for shorter tubes. This is a result of smaller current in the inner shell of shorter tubes (fig. 3.5). The nonlocal potential depends also on the tube length since the current for $x<-l_{p}$ is smaller for shorter tubes. The current decreases to zero at the end of the tube, consequently the tangent of $V_{o}(x)$ at this position has to be parallel to the $x$-axis. 


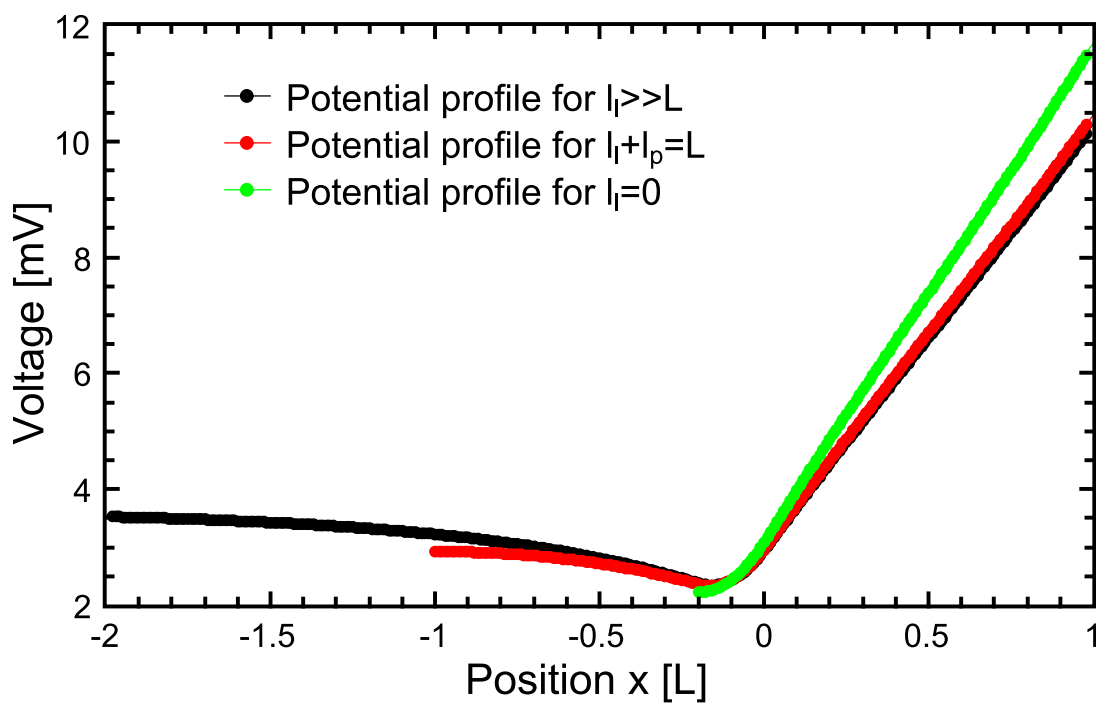

Figure 3.7: Potential profiles for three different tube lengths. Especially for small $l_{l}$ the gradient of the local potential changes drastically. The tangent at $x=-l_{l}-l_{p}$ is parallel to the $x$-axis. Same parameters as in fig. 3.5.

\subsection{Comparison of both models}

In their experiment Bourlon et al. [94] evaporated $200 \mathrm{~nm}$ broad metal electrodes separated by $200 \mathrm{~nm}$ free tube area, using two of them for current injection and two for voltage measurements. To compare his setup to one with solely two evaporated injection electrodes a model with an additional centered electrode in between was calculated. The differential equations for the third electrode are the same as that of the injecting electrodes. The boundary conditions for $I_{o}(x)$ are determined in the same manner as in section 3.2.2 for the outer pads. Since no total current flows through the center electrode the condition $I_{p}(x)=0$ holds for both ends of it.

The results for current and voltage ${ }^{1}$ are shown in fig. 3.8, calculated with continuous current injection (thick black line) and with punctual current injection ${ }^{2}$ (thin red line). For reference, the potential profile without additional electrode is also shown (green line). The same parameters were used for all calculations. The current in the outer shell is obviously strongly reduced below the metal electrodes. The current exits the tube at the beginning of the middle contact and reenters the

\footnotetext{
${ }^{1}$ Since the model with punctual current injection has no intrinsic contact resistance, the contact resistance was also not calculated for the new model.

${ }^{2}$ For the model with punctual current injection a center electrode does not change anything.
} 

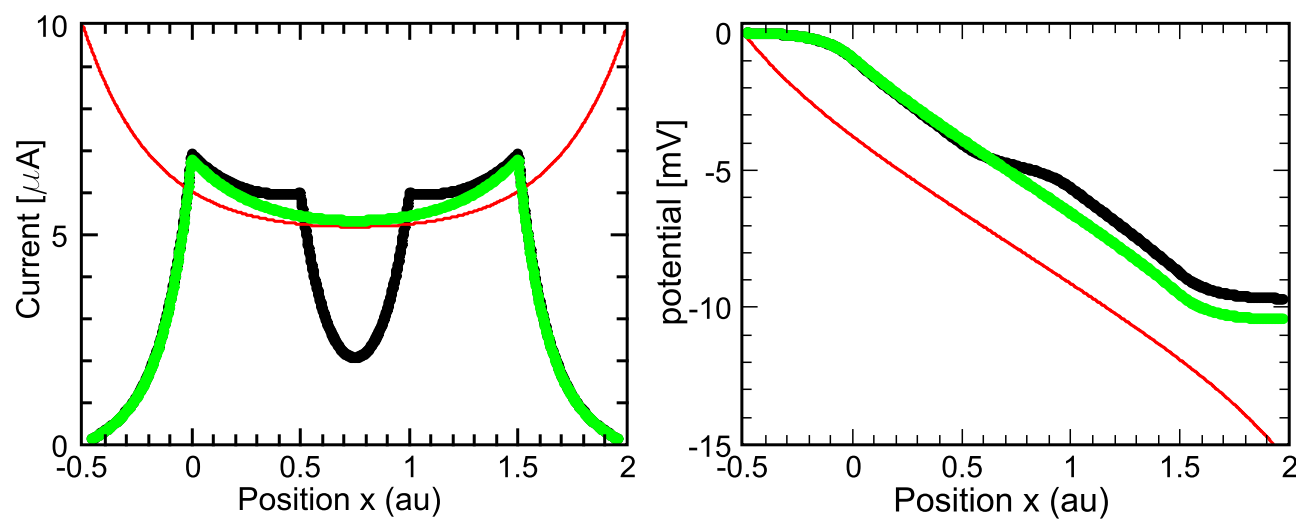

Figure 3.8: Comparison between a configuration with two contacting electrodes $(x<0$ and $x>1.5)$ (green line) and with an additional electrode in the middle $(0.5<x<1)$ (black line). The result of the model with current injection at a single point [94] is also shown (thin red line).

tube again at the other border resulting in a reduced effective resistivity of the outermost shell. Since this electrode carries a part of the current, the current in the inner shell is reduced as well. Consequently the current is more localized in the outermost shell between the electrodes, compared to a system without additional electrode or the model with punctual current injection. For multiple additional electrodes this effect obviously increases.

The slope of the voltage, which is proportional to the current, consequently is slightly higher in the area without evaporated electrodes and lower below the metal compared to the model of Bourlon et al. With a given contact conductivity, which is similar to that determined in experiments, the mean gradient of the voltage is much lower and therefore the total resistance is decreased.

Since additional electrodes needed for voltage measurements in the conventional experimental geometry are clearly invasive even in classical transport, such results have to be regarded critically. In order to measure undisturbed tubes the experimental setup needs to be improved. 


\section{Experimental Setup}

The last chapter described the influence of invasivie contact electrodes which disturb the conducting properties of a carbon nanotube even if transport occurs classically. In the quantum conductance limit (i.e. mostly at low temperatures) it was already reported that evaporated electrodes can disturb the wave function drastically up to dividing the tube in several quantum dots [71]. Therefore much less invasive voltage probes have to be used.

An interesting approach uses evaporated electrodes for current injection and MWCNTs as voltage probes. Since nanotubes are cylindrical, the contact areas are small and the probes have high contact resistance as shown in [72], and therefore are noninvasive for quantum transport, too. Furthermore, the position of the CNT probes could be adjusted by means of shifting them with an AFM [72]. Since relatively thick MWCNTs were used, the error in the lateral position is rather large.

Another approach measuring the potential profile along a nanotube is using an EFM. In this method an AFM with a conducting tip measures at a small fixed height above the sample the local electrostatic force between the sample and the tip that is set to a constant potential [60]. Since also neighboring parts of the tube contribute to the force, the lateral resolution is limited by the distance of the tip to the sample and the radius of the tip. In ref. [60] the lateral resolution is about $100 \mathrm{~nm}$.

An early experiment using movable nanocontacts was performed by Dai et al. [97]. The authors produced Au patterns using conventional lithography methods that covered parts of carbon nanotubes by accident. Using them as first electrode and the tip of a conducting AFM as second one, they measured the two point resistance versus length. Since the contact quality between tip and nanotube varies for different positions and due to the typical problem in two point measurements that it is not possible to distinguish between intrinsic and contact resistance, the uncertainty in the resistance is relatively high.

Trying to combine the accuracy of a four point measurement with the good lateral 
resolution of a conducting AFM leads to the experiment of Yaish et al [62]. They contacted a nanotube with two patterned gold electrodes used to drive a current and an additional direct contact in between the electrodes using the tip of a conducting AFM which acted as a voltage probe. With the tip moving along the tube the potential versus position can be measured. A disadvantage of this method is that the contact mode of the AFM, damages the tube by and by. Furthermore, the force pressing the tip onto the tube can induce deformations and the direct contact between tip and tube can generate Schottky barriers [62].

Here we present a new STM based technique with a higher lateral resolution than with AFM and a good accuracy in voltage measurements which works in a noncontact technique.

\subsection{Sample design}

The following approach to measure the potential profile of a carbon nanotube with a STM needs a dedicated sample design. Since the feedback loop regulates the height of the tip using the tunneling current to the sample, it cannot operate on insulating substrates. On the other hand, for electron transport measurements the substrate has to be insulating because otherwise the transport properties of the sample cannot be separated from that of the substrate.

At first view these two requirements seem to contradict each other. To solve this discrepancy, a stack of a metallic layer followed by a thin insulating layer was produced on the sample substrate. For the conducting layer tantalum was chosen because it grows with a smooth surface on highly doped silicon with thermal oxide on top. The metal was deposited via standard magnetron sputtering. The insulating layer, however, was built in a two step process. First, a thin aluminum layer of $8 \AA$ but completely covering the fresh Ta layer below was sputtered in the same chamber. Then the aluminum layer was oxidized for 15 minutes in 300 mbar pure oxygen, now forming the insulating $\mathrm{Al}_{2} \mathrm{O}_{3}$ layer. The implementation of oxygen results in an increased thickness of the layer of about $1.2 \mathrm{~nm}$.

To investigate the quality of the tunneling barrier, a square Au pattern with $30 \mu \mathrm{m}$ side length was evaporated. Contacting both, the pattern above and the Ta layer below, the $I-V$ characteristics of the $\mathrm{Al}_{2} \mathrm{O}_{3}$ barrier were recorded. A typical example for a good barrier is shown in fig. 4.1. Good barriers endured voltages up to $2.5 \mathrm{~V}$ until they were destroyed. Referring to Brinkman and Dynes [98], the tunneling characteristic can be described with a polynomial of degree 


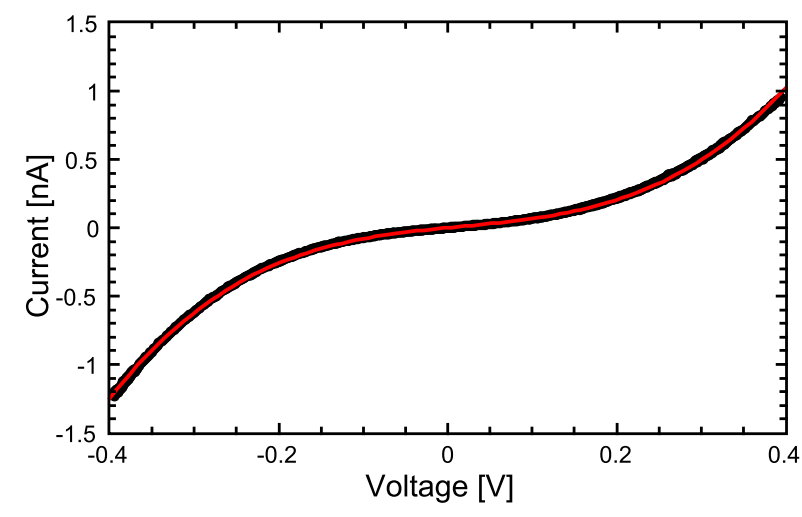

Figure 4.1: $I-V$ characteristics of a good $\mathrm{Al}_{2} \mathrm{O}_{3}$ barrier. The red curve is the fit with the formalism of Brinkman [98]. Since the bias voltage on a nanotube sample is of the order of $20 \mu \mathrm{V}$ and the evaporated area of the electrodes is below this square, the leakage current is lower than $10 \mathrm{pA}$.

three. The parameters of the tunneling process can be determined easily from its coefficients (the procedure is described in detail in ref. [99]). Based on the fit in fig. 4.1 the tunneling parameters were determined:

$$
\begin{aligned}
\text { average barrier height } & \bar{\varphi}=0.63 \mathrm{eV} \\
\text { tunneling distance } & d=2.3 \mathrm{~nm}
\end{aligned}
$$

The tunnel barrier height $\bar{\varphi}$ is lower than expected for $\mathrm{Al}_{2} \mathrm{O}_{3}$. In contrast, the value of the tunneling distance $d$ is larger than the expected $1.2 \mathrm{~nm}$. The reduced effective barrier height and the increased distance is an effect of inhomogeneities in the thickness of the insulating layer.

To generate a coordinate system on the sample, alignment marks were evaporated using lithography and lift-off techniques. The $\mathrm{Ta} / \mathrm{Al}_{2} \mathrm{O}_{3}$ bilayer was patterned subsequently in squares with a side length of $200 \mu \mathrm{m}$ using lithography and dry etching methods. This allows evaporating contact fingers that reach out of the area with the thin and sensitive insulating $\mathrm{Al}_{2} \mathrm{O}_{3}$ layer to the more stable $300 \mathrm{~nm}$ thick $\mathrm{SiO}_{2}$ covered $\mathrm{Si}$ substrate. Since these contact fingers cross the edge of the square with the exposed Ta layer in the cross-section, an additional insulating material was grown around the squares. This was $\mathrm{SiO}_{2}$ in the case of samples $\mathrm{B}$ and $\mathrm{D}$ via $\mathrm{PECVD}^{1}$ and $\mathrm{Al}_{2} \mathrm{O}_{3}$ in the case of samples $\mathrm{A}$ and $\mathrm{C}$ via $\mathrm{ALD}^{2}$.

Multiwall carbon nanotubes were suspended on the surface from a solution in orthodichlorbenzene by applying a drop of the solution on the sample and flushing it after some time with isopropanol. The nanotubes that hit the substrate stick on it due to Van der Waals forces. The positions of the tubes relative to the alignment marks were determined in a scanning electron microscope (SEM). Since

\footnotetext{
${ }^{1}$ Plasma-enhanced chemical vapor deposition

${ }^{2}$ Atomic layer deposition
} 


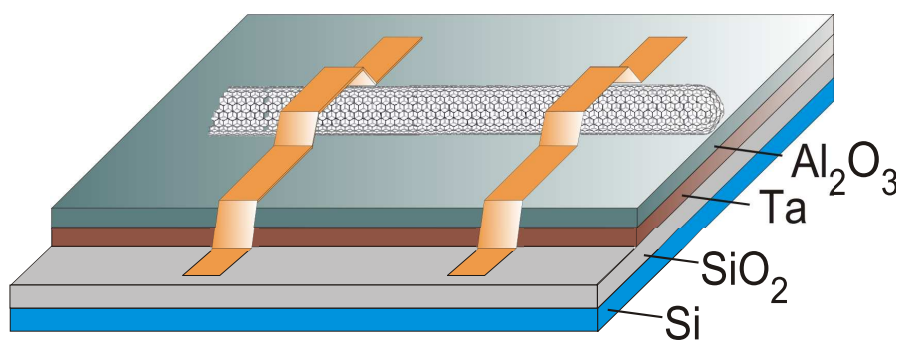

Figure 4.2: On the Si substrate with $300 \mathrm{~nm}$ thermal oxide, a stack of Ta and a thin $\mathrm{Al}_{2} \mathrm{O}_{3}$ layer was prestructured. The nanotube is contacted with $\mathrm{Pd} / \mathrm{Au}$ electrodes reaching down from the prestructured square to the $\mathrm{SiO}_{2}$ covered substrate. Unlike in the drawing, the sides of the Ta film are also covered with an insulating material.

the alignment marks are visible under the $\mathrm{PMMA}^{1}$ electron lithography resist, the contact electrodes can be defined directly on the tube using electron beam lithography. Furthermore, conductive paths out of the prestructured squares have been defined. After evaporating the metal electrodes and a subsequent lift-off step the sample is ready for use. A schematic drawing is shown in fig. 4.2.

Several experimental works revealed that transport properties of Pd contacts are superior to other metals. The contact resistance is lower $[96,100,101]$ and the formation of Schottky barriers is strongly reduced [102]. Ab initio calculations of Nemec, Tománek and Cuniberti [103] confirm these results tracing back the superiority of $\mathrm{Pd}$ to metal-nanotube hybridizations.

To benefit from the good contact properties the metal electrodes consist of a thin $(\approx 3 \mathrm{~nm}$ ) layer of $\mathrm{Pd}$, followed by a thicker Au layer in order to reduce the total resistance in the electrode. Additional bigger $\mathrm{Cr} / \mathrm{Au}$ squares adjacent to the contact lines on the $\mathrm{SiO}_{2}$ allow connecting supply lines.

\subsection{Measurement setup}

The complete sample preparation takes place under clean room conditions. The measurements, however, have been performed under clean ultra high vacuum conditions in the UHV-Nanoprobe consisting of four independent scanning tunneling microscopes (STM) (see fig. 4.3). Furthermore, a scanning electron microscope (SEM) is mounted in the vacuum chamber, required for positioning the tips on the sample. Additional features of the system are an electron spectrometer for scan-

${ }^{1}$ Poly(methyl methacrylate) 


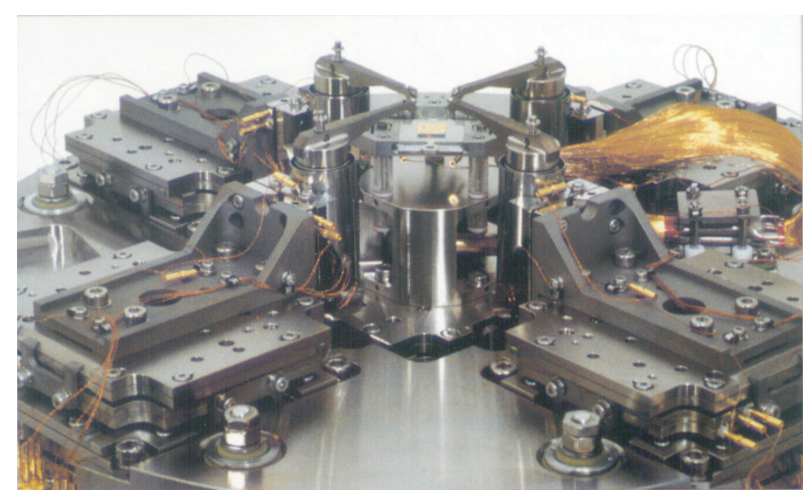

Figure 4.3: The UHV-Nanoprobe (Omicron) consisting of the sample stage in the center and the four stages for the tips. The tips are attached at the end of the cantilevers above the sample stage. All five stages are movable in $x$ and $y$ direction and the tip stages additionally in $z$ direction. The tip cantilevers are magnetically held on $90^{\circ}$ quadrant piezo tubes. The oxygen free Au-coated copper braid used for cooling is visible on the right side of the image.

ning Auger measurements and a Mott detector for scanning electron microscopy with (spin) polarization analysis (SEMPA). The attached preparation chamber was used in this work for tip preparation, but can also be used for sample cleaning via heating and sputtering and evaporating thin metal layers epitaxially.

Attention should be paid to the fact that the two imaging methods SEM and STM affect each other. The metallic tips disturb the distribution of the electric field above the sample and therefore deform the electron beam of the SEM affecting its resolution. The SEM on its side deposits electrons on a STM tip or at least secondary electrons caused by the electron beam hit the tip contributing to the total current detected by the feedback control. Since only low tunnel currents have been used in this work (see below) this current exceeds the setpoint causing setpoint detection on every height or, with the other sign, tip crashing.

For the measurements reported in this work two Au tips, attached on geometrically adequate cantilevers, have been used to contact the square contact pads on the $\mathrm{SiO}_{2}$ and in order to drive the current through the nanotube with a constant current source $^{1}$. A third tip, made of tungsten for better stiffness, was used to pierce the $\mathrm{Al}_{2} \mathrm{O}_{3}$ layer and contact the Ta layer below the nanotube. It can be used to ground the conducting layer in order to allow tunneling current to the STM tip for imaging or to apply a gate voltage in the potentiometric mode.

${ }^{1}$ Keithley 6221 

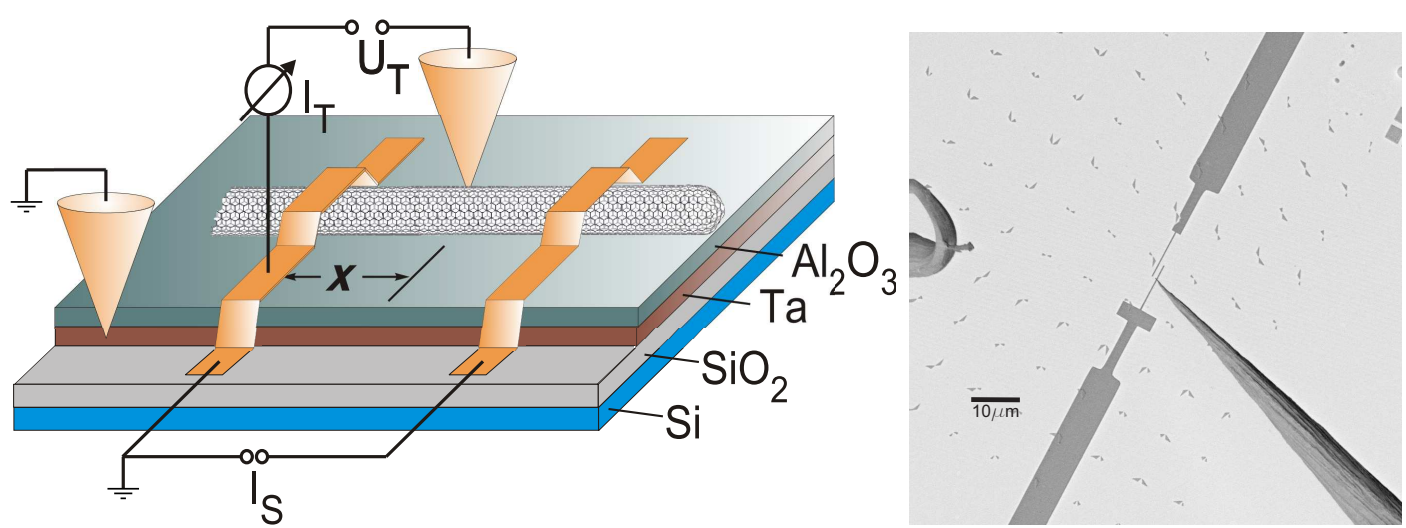

Figure 4.4: Left: A schematic drawing of the measurement setup. A current $I_{s}$ is driven through the nanotube via evaporated electrodes. The tunneling voltage $U_{T}$ is applied between the tip and one electrode and the tunneling current $I_{T}$ is measured. Right: SEM image of the sample with the tip piercing the oxide layer (on the left) and the tip used for probing the potential at the tube that is between the thin electrodes.

At the forth tip position a sharp $\mathrm{W}$ tip $^{1}$ was applied and used for imaging the tube and for the potentiometric measurements. The measurement setup is shown schematically in fig. 4.4 .

With both, the Ta layer and at least one electrode on ground potential, the STM tip was used to locate and image the tube. Since the effective tunnel barrier is the sum of the insulating $\mathrm{Al}_{2} \mathrm{O}_{3}$ layer and the gap between the tip and the surface, the feedback parameters have been chosen carefully to avoid a crash with the surface. In the experiment a tunnel voltage of $U_{T}=2 \mathrm{~V}$ and a current setpoint of $I_{T}=20 \mathrm{pA}$ were found to be reasonable. The strong increase in the tunnel current at this voltage might be an effect of image potential states at this energy [104].

After locating the tube and final positioning of the scanning area, the potentiometric measurement has been started. For this purpose the current in the tube was switched on and the tip was positioned on the tube. Switching off the feedback loop and lowering the tunnel distance a few ångström results in a better contact between tip and tube but remains noninvasive since the tunnel character persists. After taking an $I-V$ characteristic, the feedback loop was turned on again and a new position has been approached. At the zero crossing of the $I$ - $V$-curve

\footnotetext{
${ }^{1}$ All used W tips were heated in UHV conditions to at least $1000{ }^{\circ} \mathrm{C}$ to remove oxide. After this a small tip radius was confirmed with field emission current between the tip and a surface at a distance of $\approx 1 \mathrm{~mm}$.
} 
the current flow is disabled because the potential difference between tip and the position on the tube below is zero. The voltage on this balanced point is easy to read out and represents the potential of the outermost shell of the tube. Plotting against the position on the tube yields the potential profile. The lowering of the tunnel distance does not affect the measured potential [105], but improves the measurement accuracy.

This is strictly valid only in the case of diffusive transport where the electrons are nearly in an equilibrium state for every position of the tube. In this case the Fermi distribution is valid and defines the electrochemical potential. For pure ballistic transport, however, one expects two different electrochemical potentials for electrons with $\mathrm{k}$-vectors pointing from left to right and vice versa. This results because the two electron reservoirs of the electrodes can only inject electrons with k-vectors pointing away from the electrode and up to their Fermi level. Assuming highly transparent contacts and neglecting backscattering at the nanotubeelectrode interfaces results in two different electrochemical potentials for the two directions of the $\mathbf{k}$-vectors according to the potentials in the electrodes.

If impurities in the tube and backscattering effects at the tube-metal interfaces are considered, the picture becomes more complicated. A sufficient description can be achieved with the Büttiker formula [106-108]. The present setup can be described as a three terminal geometry with two contact electrodes (terminals 1 and 2) and the STM tip (terminal p). The total current $I_{p}$ in the tip which is used as a probe is the sum of the currents originating from contacts 1 and 2 . The current between two terminals $\alpha$ and $\beta$ can be written as a product of the transmission probabilities $T_{\alpha \beta}$ and the differences of the electrochemical potentials $\mu_{\alpha}$ and $\mu_{\beta}$ of the contacts, respectively. Therefore the total current in the probe is:

$$
I_{p}=\frac{2 e}{h}\left(T_{p 1}\left(\mu_{p}-\mu_{1}\right)+T_{p 2}\left(\mu_{p}-\mu_{2}\right)\right)
$$

In principle, a current flow in the probe is not necessary for voltage measurements. This can be fulfilled if the probe is floating or with a voltage compensation. For $I_{p}=0$ equation (4.1) can be solved:

$$
\mu_{p}=\frac{T_{31} \mu_{1}+T_{32} \mu_{2}}{T_{31}+T_{32}}
$$

If the probe is weakly coupled to the conductor at a single point (tunneling barrier), scattering with the lead is suppressed and the potential in the probe is independent of the strength of the coupling to the conductor and of the density 
of states in the probe [105]:

$$
\mu_{p}=\frac{\nu_{x 1} \mu_{1}+\nu_{x 2} \mu_{2}}{\nu(x)}
$$

with $\nu_{x \alpha}$ being the injectivities of contact $\alpha$ to the tube point $x$ with the local density of states $\nu(x)$. Since all terms in equation (4.3) are independent of the presence and properties of a probe, it does not disturb the intrinsic transport properties and can be used to define an electrochemical potential for each position. It should be remarked that the Fermi distribution is only valid in the probe itself, whereas the electrons inside the conductor are in a nonequilibrium state.

The contact between the STM tip and the MWCNT has typically spatial dimensions of the order of one or a few atoms and due to the STM equipment the contact can be held in tunneling state. Therefore this contact is weak in the meaning mentioned above. Unlike to commonly used voltmeters with finite impedance, the employment of the tunneling $I-V$ characteristics leads to no current at all through the probe at the zero crossing. Thus the applied voltage is equal to the potential of the probe in floating state.

In the classical limit (many scattering events which destroy phase coherence) the transmission probabilities depend mainly on the number of scattering events. Since the phase destroying electron-phonon end electron-electron scattering is not localized but equally distributed in the conductor, the distance between the probe and the contact dominates the transmission probability. In an adiabatic limit where enough scattering events occur so that the electrons are in an equilibrium state at every position, the Fermi distribution is valid in the conductor and the measured electrochemical potential is the Fermi level. 


\section{Results obtained at room temperature}

As discussed in the last chapter in principle STM based potentiometry is a noncontact method and therefore the tube is not damaged during the measurements. Since the feedback parameters have to be adjusted adequately to not crash the tip on the oxide overlayer, the tunnel conditions at the metal electrodes are not ideal. In fact the tunnel contact is so instable that several spikes in the current image appear indicating direct contact between tip and electrodes. Since the tip is charged to a voltage of $2 \mathrm{~V}$ this produces voltage peaks close to the breakdown voltage of the thin insulating spacer to the bottom electrode.

After all, the advantage over the AFM method, of not damaging the tube is counterbalanced by the disadvantage of destroying the oxide layer below an electrode. As soon as one electrode has contact to the Ta layer, voltage peaks can be diverted by this conducting channel including the nanotube, protecting the second electrode. So, only samples with a high quality insulator withstand the imaging procedure without contact to the metallic sublayer. In this work only one sample's insulating barrier rested completely intact during the measurement of the potential profile: Sample C (section 5.3). In this case the Ta layer can be additionally used as a back-gate.

In all other samples one of the injection electrodes has ohmic contact to the metallic layer. Therefore it is set to ground potential and the intact electrode is used to apply an adequate voltage. If no other value is mentioned the current through the tube was set to $I_{T}=1 \mu \mathrm{A}$. 

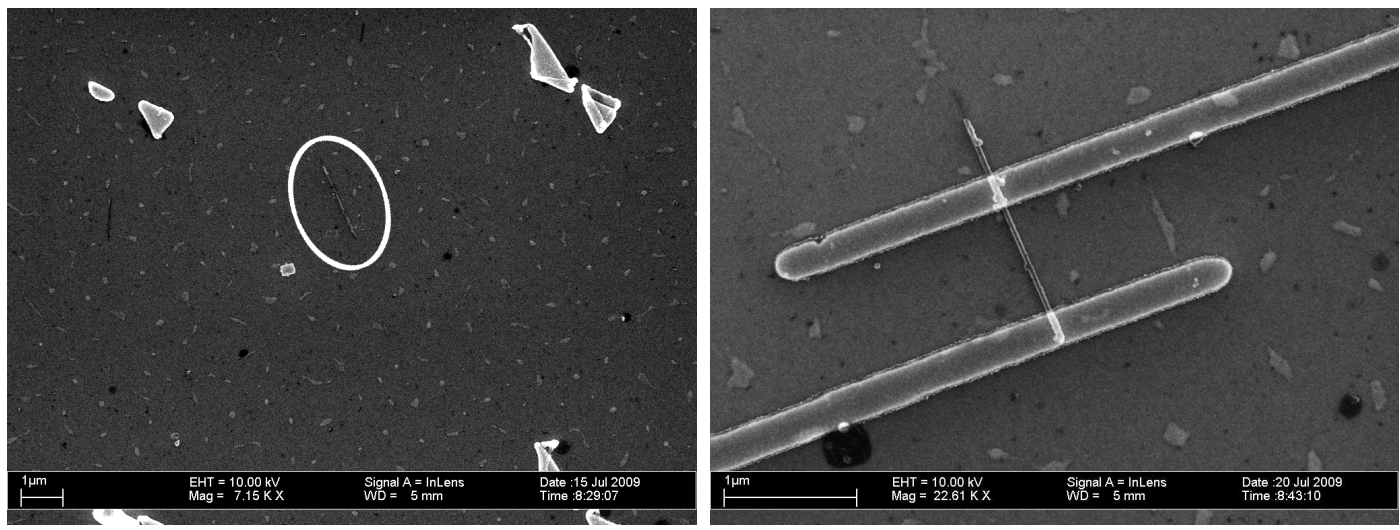

Figure 5.1: SEM images of the multiwall carbon nanotube without obvious defects (sample A). Left: The tube on the surface without electrodes. The distance of the alignment marks is $10 \mu \mathrm{m}$. Right: the same tube with evaporated $\mathrm{Pd} / \mathrm{Au}$ electrodes.

\subsection{Sample A: a multiwall carbon nanotube with no obvious defects}

A MWCNT with no obvious defect was selected and contacted with metal electrodes (fig. 5.1) as described in chapter 4. The SEM image (fig. 5.1) reveals a diameter of $d \approx 33 \mathrm{~nm}$. The metal electrodes were evaporated asymmetrically in order to leave a sufficiently long part of the tube beyond the contacts to allow local as well as nonlocal measurements.

In the STM images (fig. 5.2) the electrodes and the nanotube itself appear to be expanded compared to the SEM images in fig. 5.1. This broadening is due to the geometry of the tip relative to the sample. Since the tip and the sample surface draw an angle of about $50^{\circ}-60^{\circ}$ every structure with nonzero height results in a lateral enlargement in the STM image raked at one edge. Furthermore, due to the not perpendicular tip direction a sideways part of the tip scans the surface, where the radius of curvature exceeds that of the tip.

Additionally, since the tunnel parameters are optimized for scanning over the oxide layer, the feedback parameters are not ideal for the highly conductive metal injection electrodes and the CNT. As described above there are even short-circuit spikes in the current, causing the feedback loop to overshoot in retracting the tip. The following decrease of the sample tip distance is limited to the difference between the actual current and the set-point. This difference can be at maximum equal to the set-point which is set to a few pA. Consequently spikes appear in the 

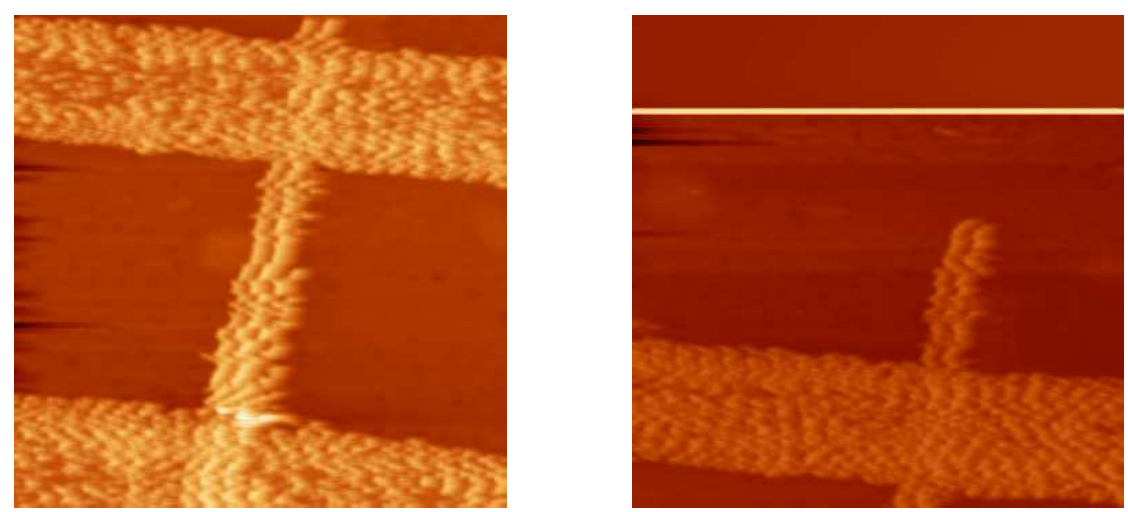

Figure 5.2: STM images of sample A between the electrodes (left) and beyond the electrodes (right).

line-scans of the topography with a high gradient at one side and a milder slope when lowering the tip again. This effect also increases the width of conducting patterns in the STM image.

Due to limitations in the scan-range the local and non-local potential measurements were done at different stage positions. To connect both potential profiles in the local as well as in the non-local measurement series, points at both sides of the electrode were measured. The combined potential profile is shown in fig. 5.3 (black filled circles) for a constant current of $1 \mu \mathrm{A}$. The curvature of the local potential is noticeable as well as the exponential like behavior of the non-local potential, both predicted by the resistivity model. For $x<0 \mathrm{~nm}$ and $650 \mathrm{~nm}<x<830 \mathrm{~nm}$ no potential variation could be observed because there the measurements were performed on the high conductive contact electrodes.

Conventional fit algorithms do not work with the huge analytic solution of the resistivity model with continuous current injection, at least with the available computer power. So another routine to fit the curves was developed. In this work a procedure similar to the simulated annealing algorithm was chosen. Starting with reasonable initial values, the parameters were varied by hand and the deviation was documented. With an understanding which parameter acts on the gradient and the bending at the different parts of the tube an adequate fit can be obtained much faster than in the purely randomly acting simulated annealing algorithm. However, in both procedures no error for the parameters can be calculated directly. It was estimated by generating fit curves with slightly higher deviations and monitoring the variation in the parameters.

With this procedure the fit in fig. 5.3 (red line) was built. The resulting parameters 


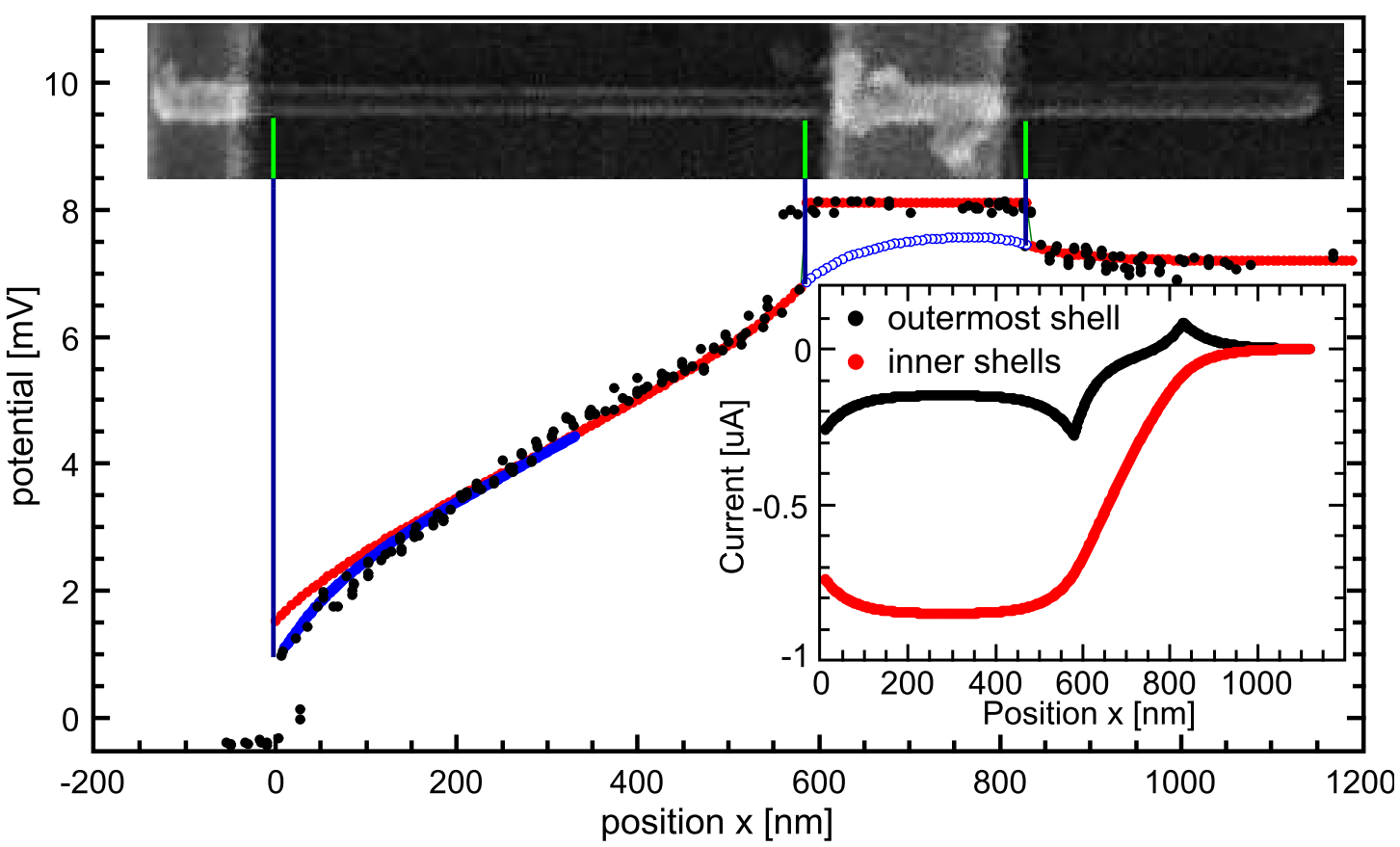

Figure 5.3: Potential profile of sample A (black filled circles). The simulation of the voltage in the outermost shell (red line) fits the measurement well, especially around the second electrode. For the left part a simulation with a smaller contact pad and a tube not exceeding the pads was performed (blue line). In the inset the current distribution of the two simulated shells in the tube is shown.

are:

$$
\begin{array}{llrl}
\text { resistivity of outer shell: } & \rho_{o} & =52 \mathrm{k} \Omega / \mu \mathrm{m} \pm 5 \mathrm{k} \Omega / \mu \mathrm{m} \\
& \text { resistivity of second shell: } & \rho_{b} & =9 \mathrm{k} \Omega / \mu \mathrm{m} \pm 3 \mathrm{k} \Omega / \mu \mathrm{m} \\
& \text { intershell conductivity: } & g & =(215 \Omega)^{-1} 1 / \mu \mathrm{m} \pm 7 \times 10^{-4} \Omega^{-1} / \mu \mathrm{m} \\
\text { contact conductivity: } & \sigma & =(147 \Omega)^{-1} 1 / \mu \mathrm{m} \pm 2 \times 10^{-5} \Omega^{-1} / \mu \mathrm{m}
\end{array}
$$

The error of $\rho_{b}$ of $30 \%$ is relatively large because this value is extracted mainly from the bending of the curve between the electrodes, whereas the approach for $\rho_{o}$ is more direct since the potential profile of the outermost shell was measured. The current that exits the part of the tube between the electrodes depends strongly on $g$ and can be extracted mainly from the potential step between the electrode and the non-local potential. The contact conductivity $\sigma$, however, shifts the potential jumps at both sides of the electrode.

The simulated curve fits the measurement well, especially for $x>200 \mathrm{~nm}$. The 


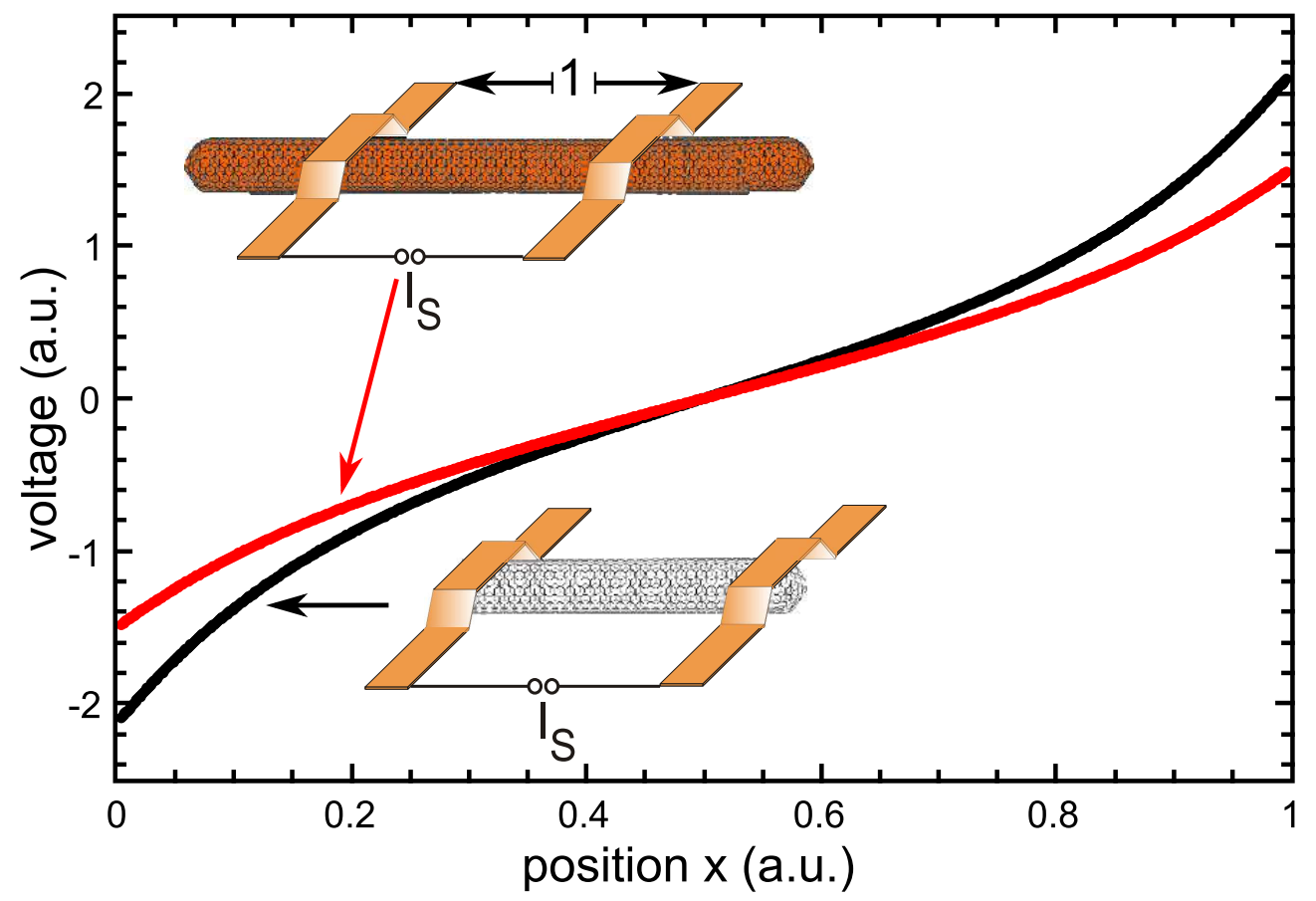

Figure 5.4: Simulations of symmetrically contacted tubes with length equal to the distance between the electrodes (black curve) and a longer tube (red curve). All other parameters are identical. The reference point for the potential is in the middle between the contacts. The potential profile of the short tube exhibits a stronger bending around the electrodes and also a higher average slope than the long tube. The insets show the geometrical configurations of the two simulations for the black and the red curve, respectively.

deviation for $x<200 \mathrm{~nm}$ is easily explained by the asymmetry of the contact electrodes as shown in fig. 5.1. The fit in fig. 5.3, however, is calculated with a model of a symmetrically contacted tube protruding from both electrodes by $420 \mathrm{~nm}$. Fig. 5.4 shows simulations of CNTs exceeding and not exceeding the region between the electrodes. This single difference in the model parameters causes a significant variation in the potential profile. Especially in the region near the electrodes the tube not exceeding the contacts exhibits a stronger bending and a significantly higher slope in the potential profile.

If one compares the fit curve with the experimental data it is clear that the fit deviates mainly around the left electrode where the tube ends below the contact. This is not astonishing because this region is not well reproduced in the symmetrical model. In this area identical modifications in the profile appear as already shown by the comparison of models of a short and a long tube. The bending is 
stronger around this position and the slope is steeper, as well. Since simulating is limited to a symmetrical model, for the left part of the tube only a model without protrusion and for the right part with protrusion applies. For the middle between both contacts, both models are appropriate. For illustration, in fig. 5.3 for the left part a simulation for a tube ending below the contacts was performed (blue line). Apart from the tube length, the same parameters were used.

Considering both, the good fit for $x>200 \mathrm{~nm}$ and the good reproduction of the measured potential for $x<200 \mathrm{~nm}$ with the model of a tube not exceeding the electrodes, it can be concluded that the resistivity model fits well to the situation in the tube.

In the resistivity model only the outermost and one inner shell is considered. Bourlon et al. [94] argued that only a small amount of current enters the second shell, and therefore even lower currents entering the inner shells can be neglected. They extracted an intershell conductivity leading to a current in the second shell of about $10 \%$ of the current in the outermost shell and correspondingly $\approx 1 \%$ in the third shell.

The simulation of the current flow in this tube, however, shows an amount of current up to $85 \%$ that leaves the outermost shell (inset of fig. 5.3). Assuming that the intershell conductivity between the second and third shell is in the same range as between outermost and second, the current in the third shell cannot be neglected any more and consequently the fit parameter $\rho_{b}$ cannot be interpreted directly as the resistivity in the second shell.

The example of this tube shows that even for shells with an extreme difference in intrashell resistivities the resulting potential profile between the electrodes is roughly linear i.e. tolerates approximation with uniform resistivity. This is shown in fig. 5.5 which compares the potential profiles of the outermost and the inner shell in the model using the parameters of this tube. Since current injection in the second shell is mainly below the electrodes and near the electrodes the behavior of its potential profile is similar to that of the outermost shell.

Including additional shells in the model changes the potential profile of the second shell only slightly since it becomes the "outermost shell of the inner tube". Consequently, the two shell model can also be used to describe tubes with multiple walls but $\rho_{b}$ cannot be directly interpreted as the resistivity of the second shell, but rather as an effective resistivity of the complete inner tube of the second shell and the shells below.

Additionally the remarkably high difference between $\rho_{o}$ and $\rho_{b}$ far beyond the 


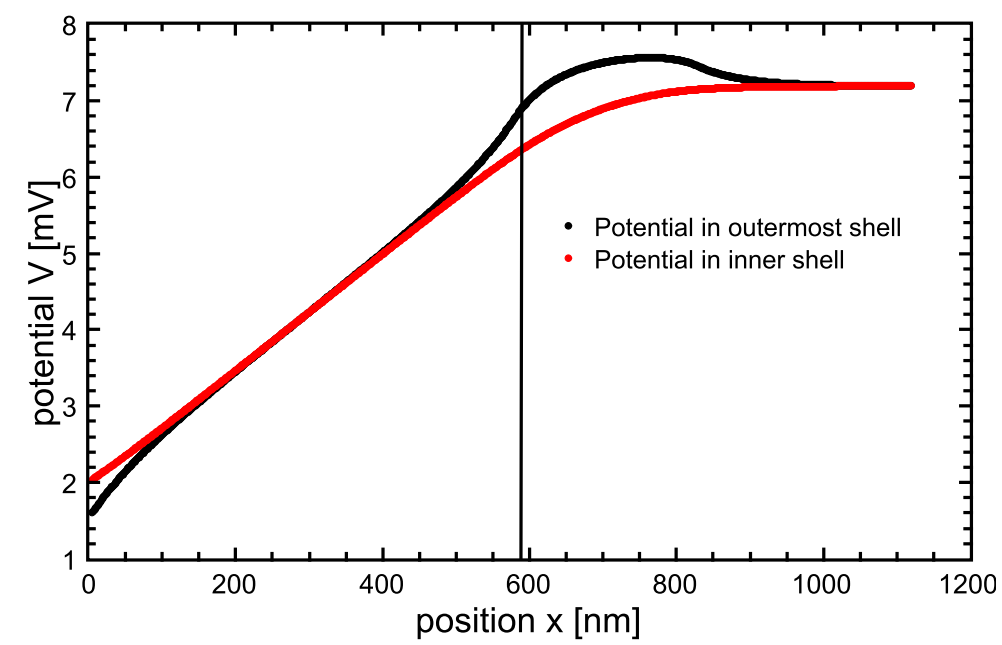

Figure 5.5: Comparison of the potential decrease in the inner and the outermost shell. Between the electrodes (on the left of the vertical line) the potential profile of the inner shell is similar to that of the outermost and both are approximately linear.

errors attracts attention. Bourlon et al. [94] explain these differences due to adsorbates or defects in the outermost shell, whereas the lower shells are protected by the outermost one. Another interpretation is that the outermost shell has a semiconducting shell ${ }^{1}$ and the inner tube can be metallic. Additionally the remarkably high difference between $\rho_{o}$ and $\rho_{b}$ far beyond the errors attracts attention. Bourlon et al. [94] explain these differences due to adsorbates or defects in the outermost shell, whereas the lower shells are protected by the outermost one. Another interpretation is that the outermost shell has a semiconducting shell ${ }^{2}$ and the inner tube can be metallic.

The intershell conductivity in our tube, however, is much higher than that determined by Bourlon et al [94]. The highest intershell conductivity determined with their method using an array of evaporated contacts is as low as $3.7^{-1} \mathrm{k} \Omega^{-1} / \mu \mathrm{m}$. Unfortunately they do not give the exact values for the diameters of their tubes but only a range in the order of about $10 \mathrm{~nm}$. To exclude the influence of the diameter, the volume resistivities ${ }^{3}$ have to be compared. The results of Bourlon

\footnotetext{
${ }^{1} \mathrm{~A}$ carbon nanotube of a diameter of $\approx 30 \mathrm{~nm}$ has a band gap of about $23 \mathrm{meV}$ [109] slightly below the thermal energy at room temperature. Consequently, charge carriers can be thermally excited.

${ }^{2} \mathrm{~A}$ carbon nanotube of a diameter of $\approx 30 \mathrm{~nm}$ has a band gap of about $23 \mathrm{meV}$ [109] slightly below the thermal energy at room temperature. Consequently, charge carriers can be thermally excited.

${ }^{3}$ As the term "resistivity" is used for resistivity of 1D materials in this work, "volume resistivity"
} 
et al. [94] show a range of $0.34 \Omega \mathrm{m}-1.8 \Omega \mathrm{m}$. Compared to the value of this tube of $0.068 \Omega \mathrm{m}$, there is still a factor 5 left. One reason for this difference are the evaporated voltage probe electrodes which localize the current in the outermost shell as mentioned in section 3.3 and therefore suppress intershell conductance.

Another explanation may arise from the difference of the diameters. For diameters larger than $15-20 \mathrm{~nm}$ polygonal cross sections have been observed [28, 110]. In this range the intershell spacings that are up to $0.39 \mathrm{~nm}$ for thin tubes decrease to nearly that of graphite of $0.34 \mathrm{~nm}$ for small tubes [111]. Both results indicate that for large diameters the tubes consist of several planar regions joined together. It can be imagined that in some of this planar regions the stacking is like in graphite. In such parts of the tube it is obvious that also the intershell coupling is comparable to that of graphite.

In the literature the volume resistivity of pure graphite in $c$-direction i.e. perpendicular to the layers spreads from $4.310^{-5} \Omega \mathrm{m}$ to $6.710^{-5} \Omega \mathrm{m}[112,113]$, depending strongly on the quality of the crystal. Even highly oriented pyrolytic graphite (HOPG) shows a volume resistivity up to 100 times higher along the $c$-axis than single crystals [113].

The volume resistivity of our tube is between the values found by Bourlon et al. [94] for smaller diameter tubes and that of graphite, indicating at least some areas with good intershell conductance. Since the value still differs from that of single crystals by a factor of $10^{3}$ an exact graphitic stacking even in small parts can be excluded. In fact, if planar regions in our tubes exist, the interlayer coupling is strongly decreased probably due to incommensurate stacking. This reduction in the interlayer coupling was also observed in twisted graphene [114].

Furthermore, for thicker tubes the 1D sub-bands are moving together giving rise to the presumption that an unavoidable doping causes more channels contributing to electron transport. Our measurements, however, reveal higher values for the intrashell resistivities. Consequently, the difference in diameter alone cannot be responsible for the discrepancy between the experimental data.

Summarizing we state that our resistivity model fits well the measured potential profile for the local as well as for the non-local potential measurements. Deviations at one side of the curve can be explained by the asymmetry of the contact electrodes. The bending of the measured potential profile between the electrodes can only be explained by a much lower resistivity of the inner tube than of the

was chosen to identify the conventional resistivity in a 3D material $\rho=\frac{E}{J}$ with the electric field $E$ and the current density $J$. 
outermost shell. Since a large part of the current leaves the outermost shell it can be assumed that non-negligible parts of the current enter the third shell and even shells further below. Consequently, $\rho_{b}$ has to be interpreted as an effective resistivity of the inner shells. Although the tube has a diameter usually yielding a polygonal cross-section, graphitic stacking can be excluded since the intershell conductivity is too low.

\subsection{Sample B: a multiwall carbon nanotube with an incomplete outermost shell}

For this sample a multiwall carbon nanotube with an incomplete outermost shell was contacted with $200 \mathrm{~nm}$ broad and $120 \mathrm{~nm}$ high $\mathrm{Pd} / \mathrm{Au}$ injection electrodes. The SEM image (fig. 5.6 (b)) shows a slight change in diameter indicating the position where has lost the outermost shell of the left side. The mean diameter is about $30 \mathrm{~nm}$. Fig. 5.6(a) shows an illustration of the two outer shells of the tube and the transition from a $n$-shell tube to a $(n-1)$-shell tube. The inner shells were left out for clarity.

The two point resistance between the electrodes was $R_{2 P}=14.4 \mathrm{k} \Omega$ which is consistent with the intrinsic resistivities for MWCNTs of $\approx 10 \mathrm{k} \Omega / \mu \mathrm{m}$ plus contact resistances of $\approx 2 \mathrm{k} \Omega$ for highly transparent contact electrodes. The potential measurement was performed for a current of $I=1 \mu \mathrm{A}$ and for $I=-1 \mu \mathrm{A}$. Since the left electrode had direct contact to the Ta layer it was set to $0 \mathrm{~V}$ for both measurements. As expected for diffusive electron transport, each potential profile is the mirrored one of the other (fig. 5.7). Both exhibit a potential jump of $\approx 1.5 \mathrm{mV}$ at the position where the outermost tube ends at $x=260 \mathrm{~nm}$. Merging the negative of the potential profile with $I=-1 \mu \mathrm{A}$ with that with $I=+1 \mu \mathrm{A}$ results in a uniform curve (open circles in fig. 5.6(c)). At $x=260 \mathrm{~nm}$ the current must eventually leave the outermost shell completely and is forced to enter lower shells. This potential step is therefore direct evidence of intershell current flow in this particular MWCNT.

Since the resistivity model in chapter 3 includes only complete shells it is improper for the situation in this tube. Furthermore, at least one additional shell has to be introduced to distinguish the part without the outermost shell from a single wall tube. Consequently, a model suitable for this tube has to include three shells at the left part and two shells at the right part of the tube. Additionally the tube is no longer symmetric, therefore for a similar model all the parts (left beyond 
(a)

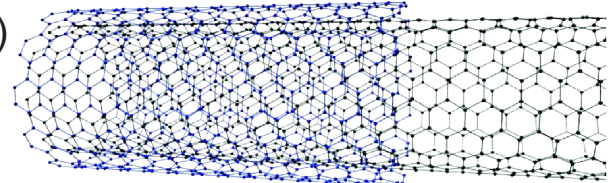

(b)

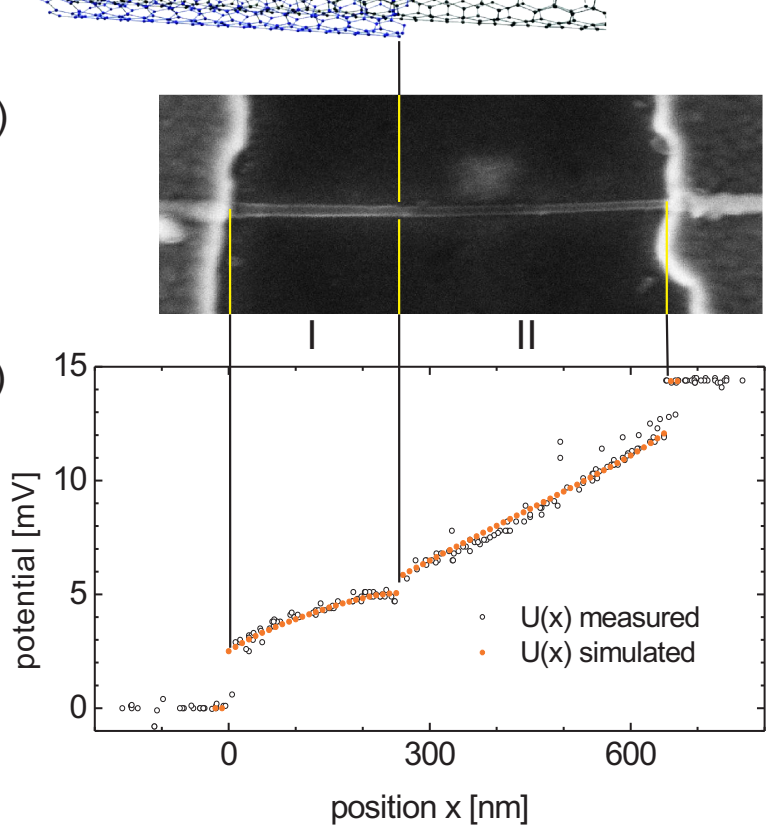

Figure 5.6: (a) An illustration of a tube with a incomplete outermost shell. Only the outermost shell and one shell below are shown. (b) The multiwall carbon nanotube between the two contact electrodes. The position where the outermost shell ends is the same as the potential jump in the measured potential profile in (c). The black open circles are the measured values and the red dots are the simulated ones.

the electrode, below the left electrode, left sector with three shells between the electrodes, right sector with two shells between the electrodes, below the right electrode and right beyond the electrode) have to be written individually in differential equation systems and connected with boundary conditions. For example, the differential equation system describing the part below the left electrode looks as follows:

$$
\begin{aligned}
& \frac{d^{2} I_{p}(x)}{d x^{2}}=-\sigma \rho_{1} I_{1}(x) \\
& \frac{d^{2} I_{1}(x)}{d x^{2}}=g\left(\rho_{1} I_{1}(x)-\rho_{2} I_{2}(x)\right)+\sigma\left(\rho_{1} I_{1}(x)\right) \\
& \frac{d^{2} I_{1}(x)}{d x^{2}}=g\left(2 \rho_{2} I_{2}(x)-\rho_{1} I_{1}(x)-\rho_{3}\left(I-I_{1}(x)-I_{2}(x)-I_{p}(x)\right)\right)
\end{aligned}
$$

with currents $I_{p}(x), I_{1}(x)$ and $I_{2}(x)$ in the electrode, first shell and second shell, respectively, the intrashell resistivities $\rho_{i}$ and the contact conductivity $\sigma$. The intrashell conductivity $g$ was assumed to be identical between the three shells. Calculating analogously for all other parts results in twelve differential equations of grade two and consequently 24 boundary conditions. Comparing this to the 


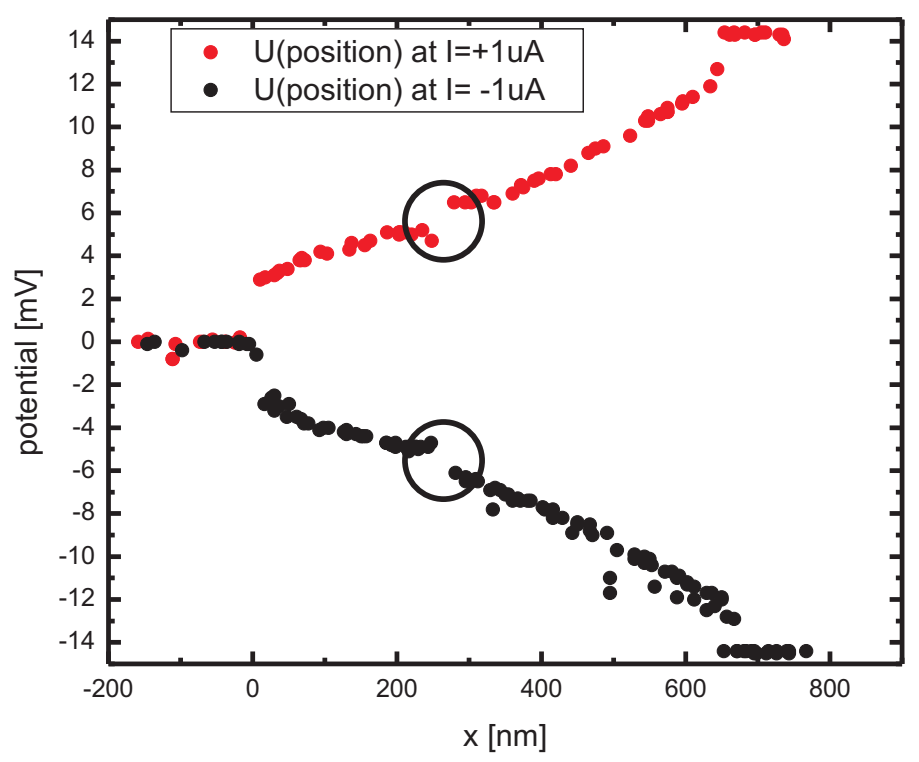

Figure 5.7: The potential profiles for both current directions. The curves have a axis of reflection at $V=0 \mathrm{~V}$. The position where the outermost shell ends is marked by the circles.

4 differential equations and eight boundary conditions in the symmetric model it becomes clear that there is no point in calculating analytically. The resistivity network was simulated numerically instead.

For this tube only the local potential between the electrodes could be measured. Therefore the ratio of the contact voltages of both sides of the electrode could not be used to determine the contact conductivity. For this reason and to reduce the fit parameters the model of punctual current injection was employed. In this configuration the contact resistances $R_{c, \text { left }}$ and $R_{c, \text { right }}$ can be extracted directly from the potential steps at $x=0$ and $x=650 \mathrm{~nm}$. For the numerical simulation a resistor network was built with three discrete resistors which describe the intraand intershell resistances of each $10 \mathrm{~nm}$ long part of the tube (fig. 5.8). The current distribution as well as the electric potentials for every node were calculated using the open source code of SPICE [115]. The fitting procedure is again similar to the simulated annealing algorithm.

If the differences between the resistivities of the outer and inner shell are due to 


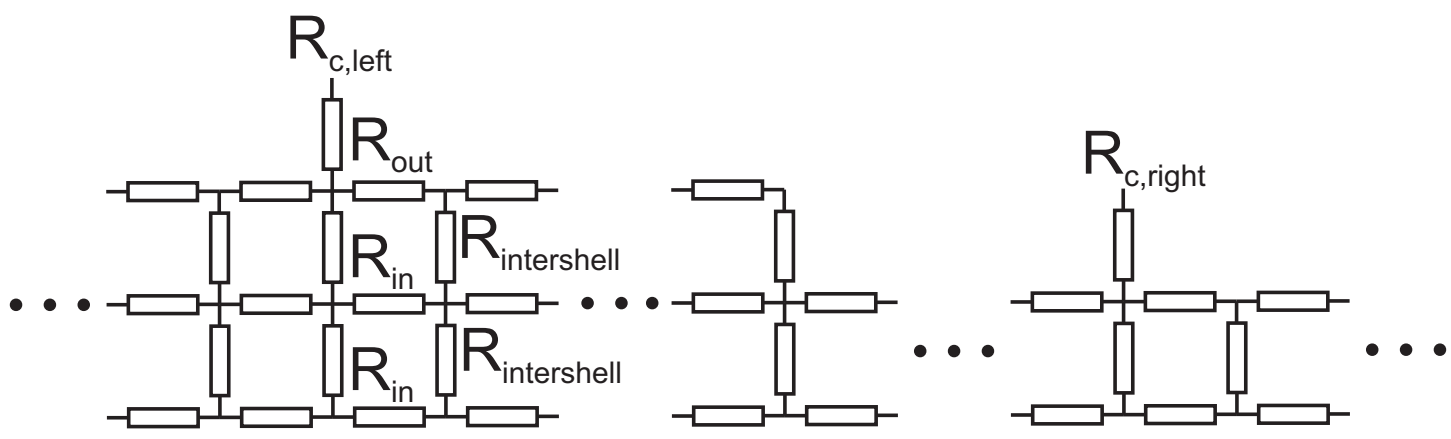

Figure 5.8: Model with incomplete outermost shell. For numerical simulation the continuous model with conductivity $g$ and resitivities $\rho_{i}$ was changed to a network of discrete resistors.

defects and adsorbates in the outermost shell, it can be assumed that only two resistivities have to be distinguished. One, $\rho_{\text {out }}$ for the respectively exterior shell that in our case is the complete outermost shell and the part of the second shell for $x>260 \mathrm{~nm}$, and $\rho_{\text {in }}$ for the second shell for $x<260 \mathrm{~nm}$ and the complete third shell. On the other hand, if we assume that the resistivities are intrinsic parameters of the three shells, three parameters $\rho_{i}$ have to be used for fitting.

We tested both possibilities and the best fit was achieved with $\rho_{\text {out }}=\rho_{\text {in }}$ and $\rho_{1}=\rho_{2}=\rho_{3}$, respectively. The results are consistent and the simulated curve is shown in fig. 5.6(c) (red dots). The complete set of fit parameters for this tube is:

$$
\begin{aligned}
R_{c, \text { left }} & =2.5 \mathrm{k} \Omega \pm 0.2 \mathrm{k} \Omega \\
R_{c, \text { right }} & =2.3 \mathrm{k} \Omega \pm 0.2 \mathrm{k} \Omega \\
g & =(100 \Omega)^{-1} 1 / \mu \mathrm{m} \pm 2 \times 10^{-3} \Omega^{-1} / \mu \mathrm{m} \\
\rho_{1} & =30 \mathrm{k} \Omega / \mu \mathrm{m} \pm 3 \mathrm{k} \Omega / \mu \mathrm{m} \\
\rho_{2} & =30 \mathrm{k} \Omega / \mu \mathrm{m} \pm 3 \mathrm{k} \Omega / \mu \mathrm{m} \\
\rho_{3} & =30 \mathrm{k} \Omega / \mu \mathrm{m}-10 \mathrm{k} \Omega / \mu \mathrm{m} /+30 \mathrm{k} \Omega / \mu \mathrm{m}
\end{aligned}
$$

The large error in $\rho_{3}$ is due to the indirect conclusion from the curvature of the potential profile mainly for $x>260 \mathrm{~nm}$. The errors of the other parameters are acceptable and the values are in the same range as those of the tube free of obvious defects. The relative error of $g$ is nearly the same as in the last tube although no non-local measurements could be done. The accuracy arises from the strong dependence of $g$ on the potential step at $x=260 \mathrm{~nm}$ that could be measured very accurately. 
Interestingly, the intrashell resitivities are equal within the experimental error and are between the values of $\rho_{o}$ and $\rho_{b}$ of the previously described tube. The high resistance of $30 \mathrm{k} \Omega / \mu \mathrm{m}$ suggests that all three shells are either semiconducting with a small band gap or have a high density of scattering centers.

Summarizing the results of this section it can be pointed out that the resistivity model provides good fits for the measured potential profile. For this purpose a numerical simulation of a resistor network had to be performed. The deduced fit parameters are consistent with those of sample A. In particular the potential jump at $x=260 \mathrm{~nm}$ was shown to be an effect of the intershell conductivity and could be measured directly.

\subsection{Sample C: A tube with a strongly varying diameter}

The sample design for sample $\mathrm{C}$ is identical with sample $\mathrm{A}$. The unique feature of this sample is a strong change in diameter at $x=270 \mathrm{~nm}$ probably due to an intramolecular junction [116]. This sample offers the possibility of back-gated measurements since both contact electrodes remained insulated from the Ta layer. The change in diameter can be observed in the SEM image (see fig. 5.9). Around the upper contact the diameter is $\approx 29 \mathrm{~nm}$ and around the lower one the diameter is $\approx 24 \mathrm{~nm}$. In potentiometric measurements $x=0$ is at the inner edge of the lower electrode. The contact resistance of the second electrode was fluctuating and not stable enough for two point resistance measurements. Nevertheless a reasonable potential profile in local geometry could be measured since the electrode with the stable contact resistance was used as voltage reference. Measurements in non-local geometry were only possible beyond this stable contact.

\subsubsection{Characteristics without gate voltage}

The potential profile (fig. 5.10) exhibits two distinct regions corresponding to the two sectors of the tube with different diameters. At the part of the tube that connects to the well defined contact local as well as non-local measurements could be performed. These measurements can be fitted well with the resistivity model 


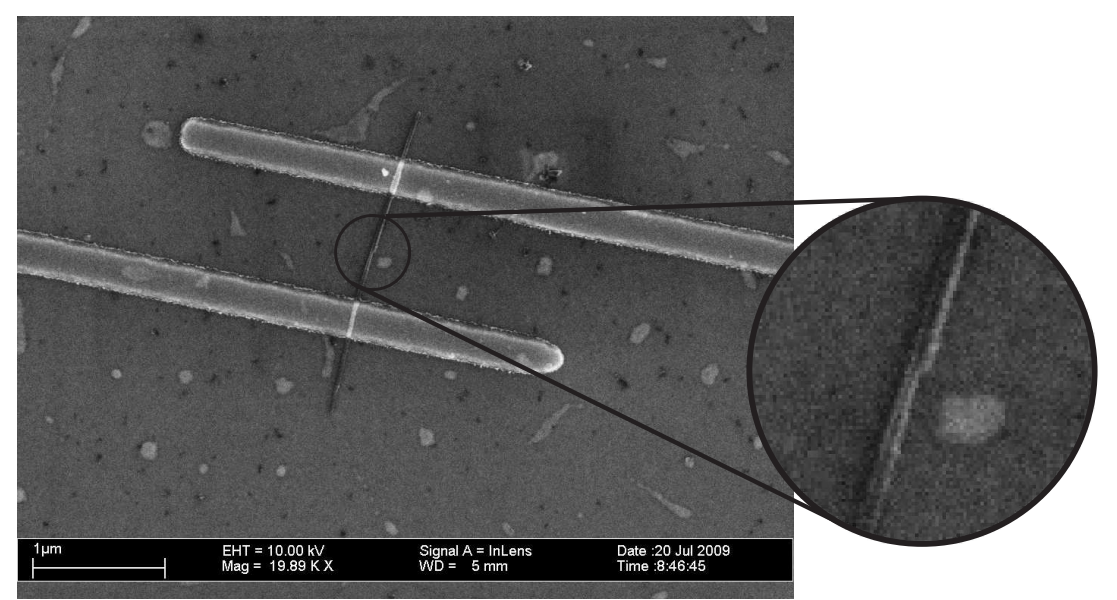

Figure 5.9: SEM image of sample C: the radius of this tube exhibits a change in diameter between the electrodes. This part is enlarged to show the transition in detail.

(red line in fig. 5.10). The fit parameters are:

$$
\begin{aligned}
\rho_{o} & =47 \mathrm{k} \Omega / \mu \mathrm{m} \pm 5 \mathrm{k} \Omega / \mu \mathrm{m} \\
\rho_{b} & =40 \mathrm{k} \Omega / \mu \mathrm{m} \pm 8 \mathrm{k} \Omega / \mu \mathrm{m} \\
g & =(1033 \Omega)^{-1} 1 / \mu \mathrm{m} \pm 2 \times 10^{-4} \Omega^{-1} / \mu \mathrm{m} \\
\sigma & =(200 \Omega)^{-1} 1 / \mu \mathrm{m} \pm 5 \times 10^{-5} \Omega^{-1} / \mu \mathrm{m}
\end{aligned}
$$

The relative errors, especially for $g$, are larger than for the samples above. This is due to the shorter part where the model is appropriate. The intrashell resistivities and the contact conductivity are in good agreement with that of samples A and B. The intershell conductivity, however, is smaller than in the samples presented above by a factor of $7-10$. This is manifested in the slight bending of the potential profile near the electrode and by the slow decrease in the gradient of the non-local potential.

Since the intershell conductivity and the difference in the intrashell resistivities of this sample are lower than those in sample A the current does not concentrate as much in the inner shells (inset of fig. 5.10). Nevertheless in the middle of the tube the currents in the outermost and the inner shell are in the same range. Therefore for this set of parameters $\rho_{b}$ has to be interpreted as the effective resistivity of the complete inner tube, too, meaning that the real resistivity of the second shell is larger than calculated. 


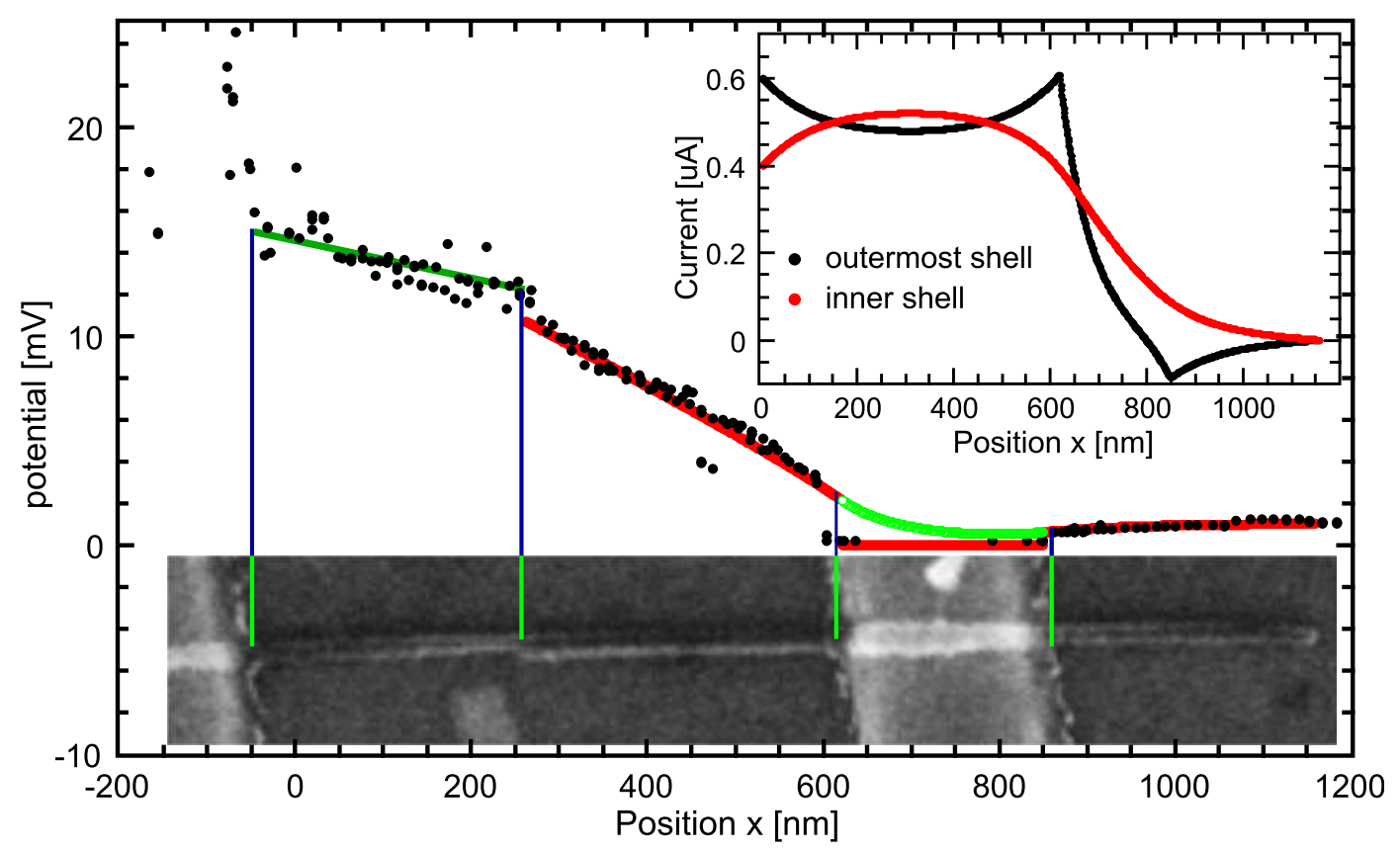

Figure 5.10: Potential profile of sample C. The right electrode with a stable contact resistance was used as voltage reference. The calculated curve (red) fits well the measurement for $x>270 \mathrm{~nm}$ that represents the thicker part of the tube. At the transition from small to big diameter a potential step occurs followed by a lower slope for the thinner tube (green line). The unstable contact conductivity at the left contact causes the potential variation for $x<0$. The inset shows the current distribution in the outermost and the inner tube within the model.

For the sector connecting to the right contact no non-local potential measurements could be performed due to the fluctuating contact resistivity. Therefore and due to the bad signal to noise ratio for $x<270 \mathrm{~nm}$ a reasonable fit for the left part of the tube was not possible. For a rough estimate of the main difference we can use the mean gradient (green line in fig. 5.10) that is much lower than for the thicker sector corresponding to a mean resistivity of $10 \mathrm{k} \Omega / \mu \mathrm{m}$ compared to a mean resistivity of $24 \mathrm{k} \Omega / \mu \mathrm{m}$ of the right sector. Assuming a similar defect density in the crystal lattice in both sectors, we conclude that at least one of the shells must have a transition from a metallic to a semiconducting state. The anomaly at the defect position can be due to a Schottky barrier. This hypothesis could be tested by reversing the current direction and monitoring potential changes in the potential profile at the junction point [116]. As at first we decided to measure the gate dependency of this tube and the sample did not survive that measurement, the reversed current behavior cannot be tested anymore. 

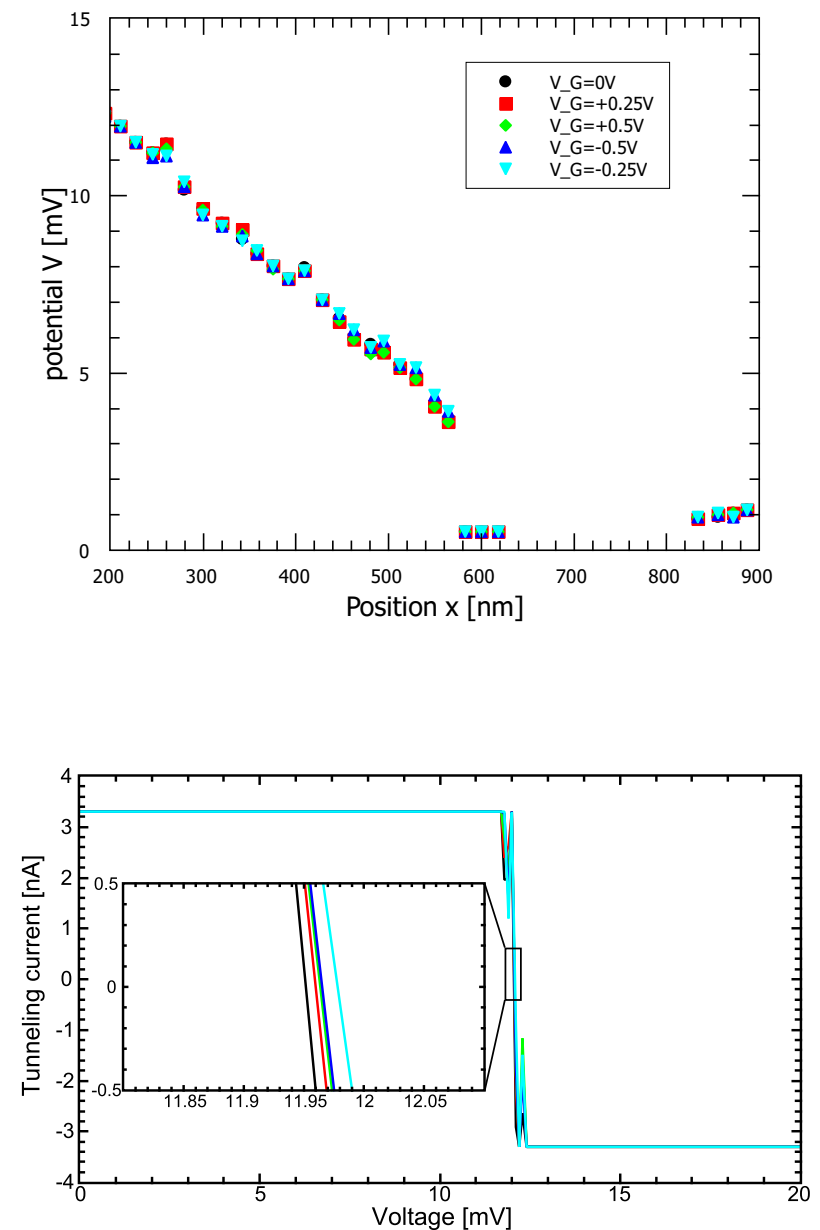

Figure 5.11: Potential profiles of sample $\mathrm{C}$ with applied gate voltages indicated by color and symbol. The potential profile is shown only for the stable thicker part of the tube.

Figure 5.12: Characteristics at one position with different gate voltages $V_{g}$ (same colors as in fig. 5.11). Due to the small tunneling distance the amplifier is in over range except near the zero crossing. The potential (voltage at $I_{t}=0$ ) does not depend measurably on the applied gate voltage. The extracted values range from $11.95 \mathrm{mV}$ to $11.98 \mathrm{mV}$.

\subsubsection{Behavior with applied gate voltage}

Applying a gate voltage on the Ta layer with respect to the electrodes results in a shift in the Fermi level of the tube. Since the Fermi level crosses the band structure at a different energy, the quantity of the contributing sub-bands can change. This affects the resistivity of the conductor in case that the conductivity is limited by the available charge carrier density.

For this experiment the STM tip was positioned on the tube and then five spectroscopy curves were recorded consecutively. Simultaneously, the tip connected to the Ta-layer was used to apply synchronously a step function with the five gate voltages in fig. 5.11. Consequently for each position the electrochemical potential with five different gate voltages could be determined. Between the measured potential profiles (fig. 5.11) no clear differences have been detected. Even at position $x=210 \mathrm{~nm}$ that is far from the voltage reference at the right contact the 

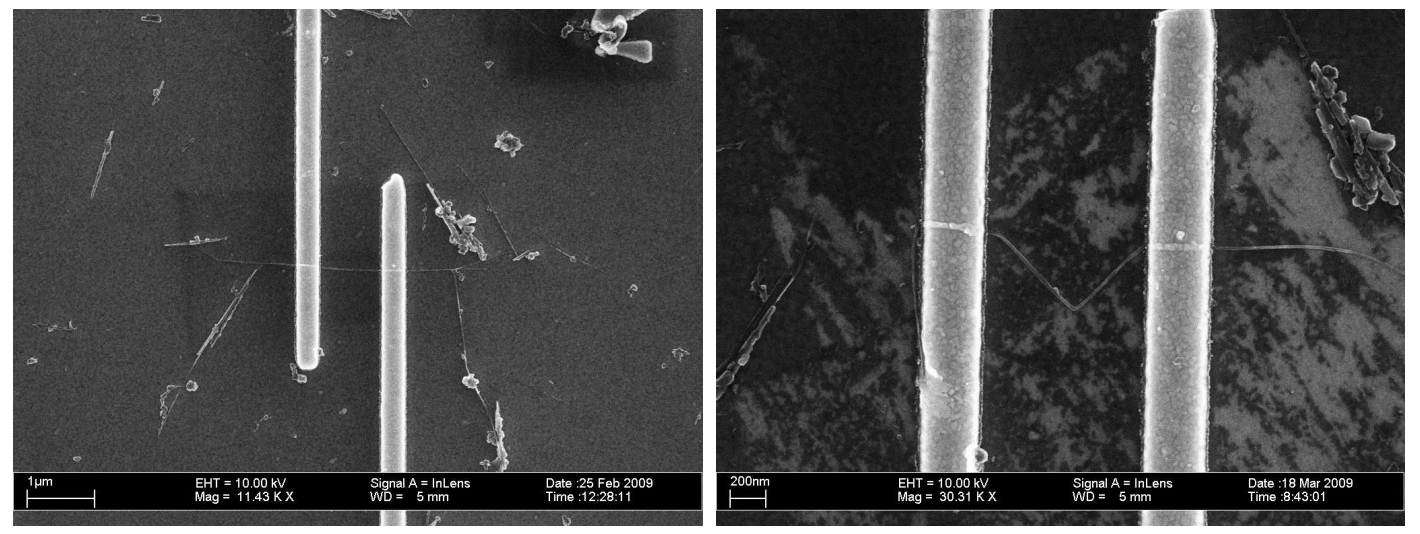

Figure 5.13: Sample D before (left) and after (right) the manipulation with the STM tip and a subsequent cleaning step in an ultrasonic bath. The originally straight tube was obviously deformed at several positions: the V-structure between the contacts that induces three kinks, an additional kink at the outer side of the left contact and a slight bending at the right part of the tube.

extracted voltages are equal in terms of differences being below the experimental error $^{1}$.

Unlike other experiments with similar tube material [54] no dependence on the gate voltage could be observed at room temperature.

\subsection{Sample D: a stretched nanotube with a kink}

The sample design of sample D is similar to sample A. Originally it was a $4 \mu \mathrm{m}$ long MWCNT with uniform diameter of $19 \mathrm{~nm}$ and without any obvious defects (left image of fig. 5.13). Due to dirt on the sample surface ${ }^{2}$ that increased the tunnel gap, a first attempt of imaging the tube with the STM failed. Because of the decreased tunnel current the feedback look caused the tip to operate too close to the surface or to actually scratch over the surface. The scan direction was perpendicular to the tube axis. During scanning operation the two-point resistance increased abruptly and irreversibly from $21 \mathrm{k} \Omega$ to $49 \mathrm{k} \Omega$ indicating plastic deformations in the tube $[84,87]$. A subsequently taken SEM image (right part of fig. 5.13) revealed the structure of the MWCNT after this accidental manipulation.

The $\mathrm{V}$-structure between the contacts is obviously longer than the original linear

\footnotetext{
${ }^{1}$ In case of these spectroscopy curves the digital steps are $80 \mu \mathrm{V}$.

${ }^{2}$ Probably residue from the EBL resist.
} 
structure, namely $\approx 300 \mathrm{~nm}$. Comparing the total length of the tube before and after manipulation reveals an elongation of $130 \mathrm{~nm}$. Additionally, the deformation of the left electrode (upper inset of fig. 5.14) indicates that the tube moved under the contact during stressing [117]. In contrast, on the right contact no deformations could be observed. Consequently the remaining length arises from the left protruding part of the tube that was pulled through below the contact.

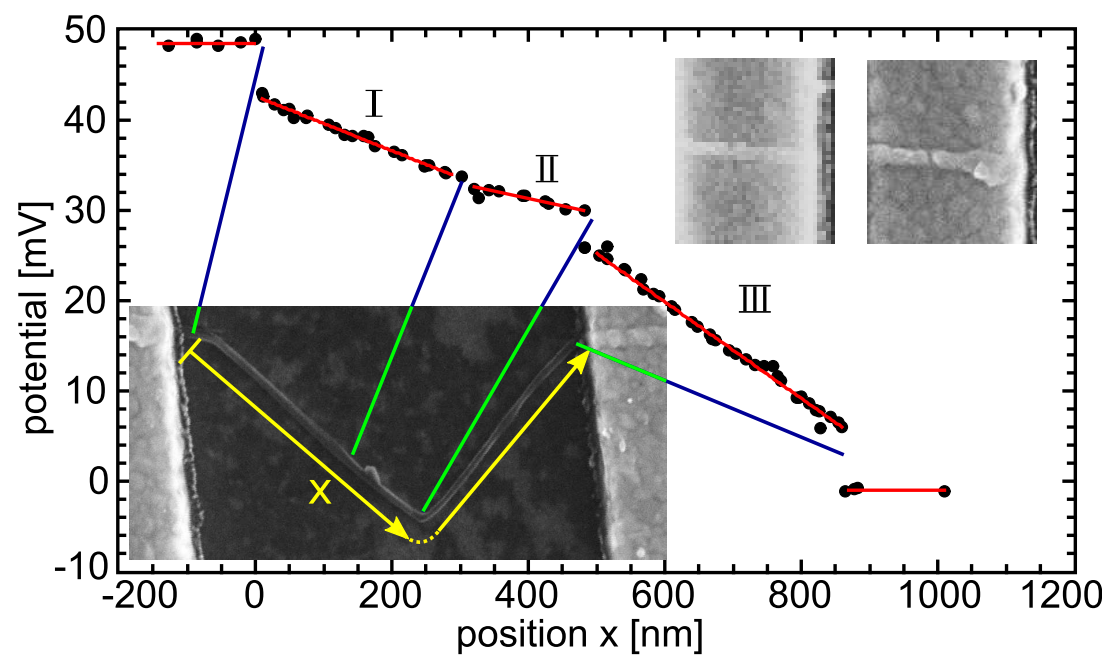

Figure 5.14: The $x$-axis of the potential profile of sample D follows the tube axis and therefore has a kink at $x=490 \mathrm{~nm}$. The profile can be segmented in three sectors corresponding to the modifications of the tube. At $x=300 \mathrm{~nm}$ the tube is narrowed from a diameter $d \approx 19 \mathrm{~nm}$ to $d \approx 14 \mathrm{~nm}$. The kink at $x=480 \mathrm{~nm}$ causes a strong increase in the slope of the potential profile. The resistivities are $30 \mathrm{k} \Omega / \mu \mathrm{m}, 15 \mathrm{k} \Omega / \mu \mathrm{m}$ and $53 \mathrm{k} \Omega / \mu \mathrm{m}$ for parts I, II and III, respectively. The upper inset compares the left electrode before (left) and after (right) manipulation.

The part between the contacts can be segmented in three sectors (fig. 5.14). The first sector ( I ) has an unaltered diameter of $19 \mathrm{~nm}$. The diameter of sectors II and III, however, was decreased to $14 \mathrm{~nm}$ during manipulation. Since only these two sectors are affected visibly, the tube elongation can be assigned entirely to them. The corresponding strain in these regions is 20\%. Elongations of this magnitude and above were already observed in singlewall nanotubes [87]. They can be theoretically described as formation of (5-7-7-5) defects via rotation of a covalent bond with followed plastic flow. The (5-7-7-5) defect splits due to repeated subsequent bond rotations in two (5-7) defects which glide around the tube and leave a tube structure in between with different chiral vector and smaller diameter (ductile behavior) [34, 83, 118]. 
Another assumption, that the MWCNT is telescopically extended, is contradicted by the lengths of the different diameters. If the tube is telescopically extended, the sector with the original diameter should be equally long as the distance between the contacts. In fact, only sector I has the original diameter and it has a length of only $490 \mathrm{~nm}$ compared to the length of the not manipulated tube of $920 \mathrm{~nm}$.

The locally measured potential profile (fig. 5.14) exhibits three different slopes corresponding to the $\mathrm{V}$-structure. The resistivity does not scale directly with the diameter. Parts II and III have similar diameters but exhibit strongly differing slope values. The kink separating part II and III induces a potential step indicating a tunnel barrier $[119,120]$. Although part II is narrowed and therefore obviously defective its resistivity is closer to the expected value before manipulation than the other parts. For this, based on the two-point resistance, a maximum of $20 \mathrm{k} \Omega / \mu \mathrm{m}$ can be extracted if the contact resistances are considered to be below $1 \mathrm{k} \Omega$. Considering reasonable contact resistances, the original resistivity can be estimated to $\approx 16 \mathrm{k} \Omega / \mu \mathrm{m}$. Parts I and III have a highly increased resistivity up to a factor of 3.5 .

The potential steps at the edges of the contacts are now larger than for the previous samples. This is a result of the increased tube resistivity at the border area below the contact which shifts the injection zone to the middle of the contact. Therefore, the resistance increase is mainly caused by a change in the intrinsic tube resistivity. The left contact resistance where the tube was pulled out has not changed as dramatically as reported by Paulson et al. [117]. From their experiments they concluded that the resistance changes during manipulation are dominated by the contact resistances whereas with the present experimental setup the resistance enhancement can be assigned to the nanotube itself.

Several mechanisms causing the increased resistivity are imaginable. Since sector I was not thinned during manipulation, plastic deformations can have been embedded only sparsely. Nevertheless, a potential gradient much higher than in the original tube was measured. If there are a few individual but large defects in the tube lattice they would be visible as voltage steps in the potential profile [79]. Such steps could not be observed.

Another possibility for the increased resistivity is a remaining elastic strain in the nanotube. In this case the friction between the tube and the $\mathrm{Al}_{2} \mathrm{O}_{3}$ surface has to be high enough to retain the strain in the nanotube. Jang et al. [85] reported that in this elastic case the resistivity can reversibly increase by up to $30 \mathrm{k} \Omega / \mu \mathrm{m}$. For this resistance increase a strain of only $3 \%$ had to be applied. Considering the resistance increase of $\approx 15 \mathrm{k} \Omega / \mu \mathrm{m}$ of sector I and using a linear approximation 
for the correlation between strain and resistivity increase, an elongation of about $1.5 \%$ has to be expected. This corresponds to only $4.5 \mathrm{~nm}$, which is negligible compared to the total elongation of $130 \mathrm{~nm}$, so the presumption that the whole elongation occurred in sectors II and III is still valid. The measured resistivity increase is also reasonable considering a study using singlewall carbon nanotubes where reversible resistance changes over two orders of magnitude were observed while straining and kinking them by means of applying a force perpendicular to the tube axis [86]. A subsequent study by Cao et al. [121] showed that a change in resistance of at least one order of magnitude can be achieved without inducing a kink in the tube.

The deformation in sectors II and III, however, is undoubtedly plastic since the deformation can be observed in the narrowed diameter. Both potential slopes of these sectors are uniform over the whole sector length indicating a high defect density and not only a few defects with large influence on the conductance. This is conform with the uniform diameter in these sectors. A similar conclusion was drawn from an experiment with an analogically kinked and stretched tube where, by means of applying a local gate (scanning gate microscopy) a uniform reaction was observed for the whole stretched part of the tube [87]. This indicates a uniform defect density. Calculations on deformed carbon nanotubes including plastic deformations caused by strain reveal a reduced density of states around the Fermi level $[82,83,122]$. Additionally, the high defect density increases scattering events. Both, the reduced availability of charge carriers and the decreased mobility give rise to a reduced conductivity in the tube.

The part of the tube outside of the contacts is not altered during manipulation and therefore should exhibit original properties. Nevertheless, the modification between the contacts influences the non-local potential since it defines how the current spreads over the shells. A large defect density increases scattering events and therefore the probability of scattering into a neighboring shell. It was also shown that broken bonds can rearrange with other shells increasing the intershell conductivity [31]. Consequently, it is not completely surprising that the non-local potential is increased compared to samples A-C, indicating a larger amount of current flowing in the inner shells (fig. 5.15).

With the protruding part of this tube being nearly twice as long than the distance of the two contacts, it is sufficiently long to neglect the disturbance of finite tube length. This is supported by the vanishing current in in the black curve of fig 3.5 at $x=-2 \mathrm{~L}$. Also, beyond the contacts the finite contact width does not influence the potential decay. Therefore the non-local potential profile can be fitted with 


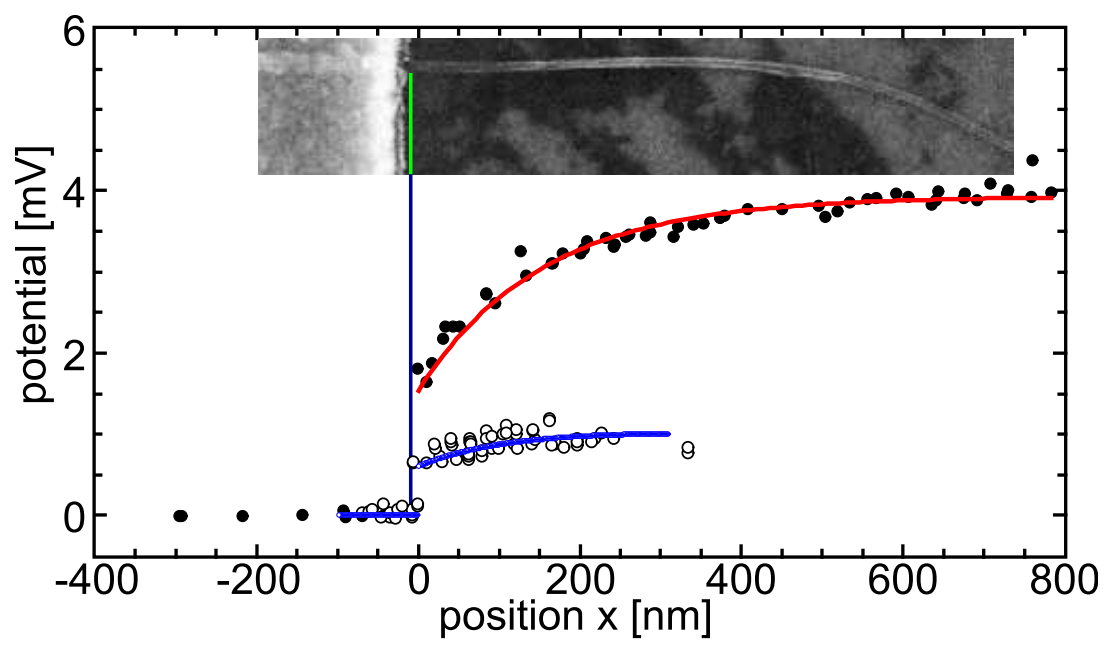

Figure 5.15: Non-local potential profile of sample D (black filled dots) compared to the non-local voltage of Sample A (open dots), each with the according fit. In case of Sample D, the tube is long enough $\left(\gg L_{a}\right)$ that it behaves purely exponentially (red line) as predicted by the model for long tubes.

a purely exponential decay similar to the easier model (equation 3.21). Consequently, the decay length $L_{a}=\left(\sqrt{g\left(\rho_{1}+\rho_{2}\right)}\right)^{-1}$ is an intrinsic property of the protruding part of the tube. The exponential fit determines $L_{a}=154 \mathrm{~nm}$ (red line in fig. 5.15). This value is in the same range as that of sample $\mathrm{C}$ which can be calculated to $L_{a}=110 \mathrm{~nm}$. Comparing this decay length with that reported by Bourlon et al. [94] reveals a difference of about one order of magnitude. Considering that the intershell conductance $g$ enters into $L_{a}$ with the square root, this is in agreement with the difference in $g$ for samples A, B and C that is up to two orders of magnitude.

The other two parameters that correspond to the shift in $x$ and $V$ direction cannot be used to extract further physical properties. The potential offset depends on the one hand on the amount of current $J$ that leaves the zone between the contacts in the inner shells and on the other hand on the voltage reference. Both, $J$ and the potential difference between the tube below the contact and the contact itself cannot be extracted from the non-local potential profile. Furthermore this parameter can easily be extracted from the horizontal asymptote and locked for the fitting procedure allowing a more accurate fitting with only two free parameters. The shift in $x$ direction is also a consequence of the changed voltage reference compared to equation 3.21. In the experiment the voltage reference is the contact electrode, whereas the model with punctual current injection neglects the contact 
resistance and sets the voltage reference on the tube at $x=0$.

In conclusion, the results of sample D show that MWCNTs can sustain a strain of $10 \%$ without rupture. Furthermore they retain a conducting state, but their conductance decreases significantly. In case of this sample, the resistance increase could be attributed almost completely to the intrinsic resistance of the tube and not to the contact resistance. The resistivity remains constant over each sector i.e. resistance is not dominated by large individual defects, but by an uniform defect density. The non-local measurements confirm the results of the previous samples, especially of the high decay length $L_{a}$. 


\section{Low temperature results}

For one sample (sample D) it was possible to additionally perform low temperature measurements. The cryostat in the UHV-Nanoprobe is connected to the sample via an oxygen free copper braid. With this design temperatures down to $110 \mathrm{~K}$ and $28 \mathrm{~K}$ can be achieved using liquid nitrogen and helium as refrigerant, respectively.

After cooling down, the temperature of the sample stage was kept constant for at least one hour in order to achieve a uniform temperature distribution on the sample. Nevertheless during the STM imaging the sample drifted with respect to the STM tip giving rise to a distorted STM image. On the one hand this is due to cooling in the stage system and on the other hand it is due to temperature variation of the tip and its cantilever. In particular, the latter reason causes additional inconveniences in the spectroscopy mode. During the STM imaging, temperature induced shortening of the tip is immediately readjusted by the feedback loop. In the spectroscopy mode, however, the feedback loop must be switched off. Furthermore the tip is additionally approached to the sample a few $\AA$ to keep the tunnel contact of the tip to the sample. Consequently the thermal conductance is also increased. Further cooling of the tip and thus the temperature induced shortening reduces the coupling to the nanotube and, due to the non perpendicular geometry, the lateral position of the tip will be changed. Therefore, integration time in the spectroscopy mode is limited and the voltage resolution is reduced compared to the room temperature measurements. The uncertainty in the voltage is $\approx 0.3 \mathrm{mV}$. Because of the drift, the nanotube position relative to the coordinate system of the STM varied during measurement. In order to position correctly on the tube, the spectroscopy curves were recorded during STM imaging. The real position of the tube was extrapolated from the previous line-scans whereas in the active line-scan the tip was stopped above the tube, the spectroscopy curve was taken and then the line-scan was continued. Since the tube of sample D is kinked between the contacts the drift gives rise to a nonuniform distortion for the sectors of different direction. Considering a constant drift rate for the whole STM image and the displacement of an image relative to the last one, we corrected the drift induced distortion. 


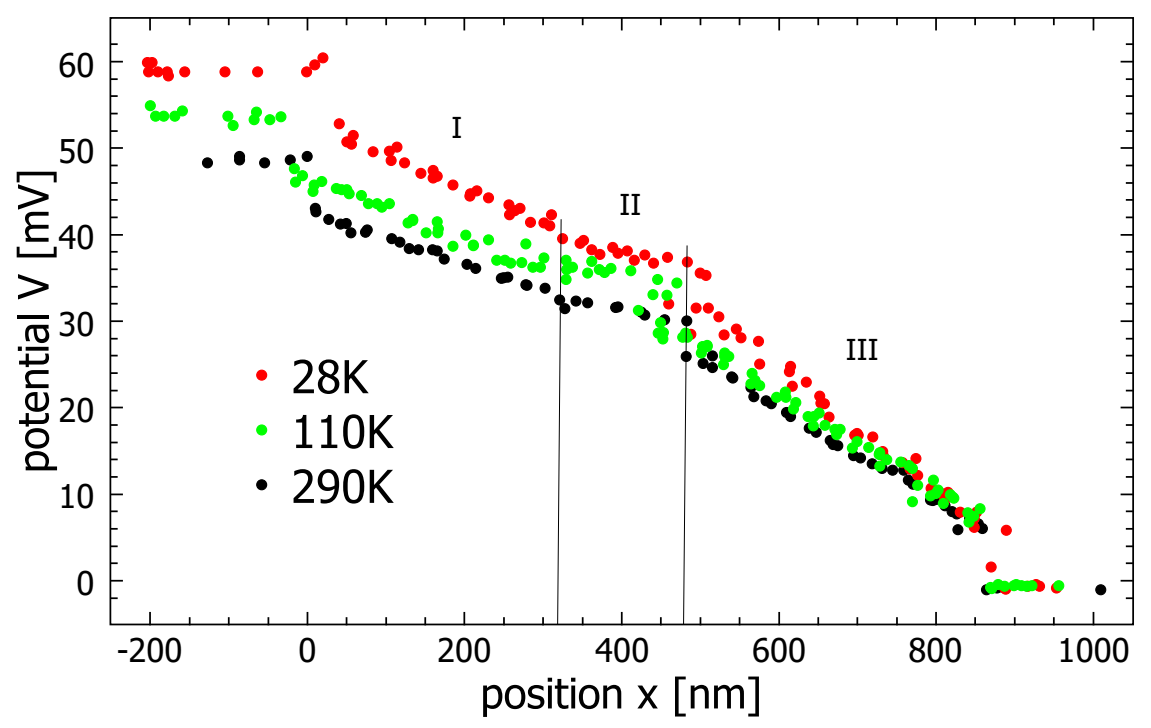

Figure 6.1: Low temperature potential profiles of sample D in local geometry. The slope of all three sectors increases with decreasing temperature whereas the voltage steps at the edge of the contacts remain approximately constant. The total resistance increases from $49 \mathrm{k} \Omega$ at room temperature to $59 \mathrm{k} \Omega$ at $28 \mathrm{~K}$.

\subsection{Measurements in local geometry}

Sample D exhibits three sectors between the contact electrodes with different behavior due to modifications in the lattice structure which occurred during manipulation (see fig. 5.14). The potential profiles of all three sectors of this sample, measured at various temperatures (fig. 6.1), show an increasing resistivity with decreasing temperature for all sectors. In particular, in sectors I and III only a slight resistivity increase was observed for the temperature step of room temperature to $110 \mathrm{~K}$ whereas for the further step down to $28 \mathrm{~K}$ a higher resistivity increase was observed. The mean slopes of all three sectors are shown in table 6.1.

Table 6.1: The resistivities of sample D extracted from the slopes in the potential profiles.

\begin{tabular}{|lccc|}
\hline & sector I & sector II & sector III \\
\hline room temperature & $30 \mathrm{k} \Omega / \mu \mathrm{m}$ & $15 \mathrm{k} \Omega / \mu \mathrm{m}$ & $53 \mathrm{k} \Omega / \mu \mathrm{m}$ \\
$110 \mathrm{~K}$ & $34 \mathrm{k} \Omega / \mu \mathrm{m}$ & $15 \mathrm{k} \Omega / \mu \mathrm{m}$ & $55 \mathrm{k} \Omega / \mu \mathrm{m}$ \\
$28 \mathrm{~K}$ & $40 \mathrm{k} \Omega / \mu \mathrm{m}$ & $17 \mathrm{k} \Omega / \mu \mathrm{m}$ & $70 \mathrm{k} \Omega / \mu \mathrm{m}$ \\
\hline$R(28 \mathrm{~K}) / R(290 \mathrm{~K})$ & 1.33 & 1.13 & 1.32 \\
\hline
\end{tabular}


This unusual temperature dependence for a metallic conductor is well known in CNTs. The most common interpretations of the increasing resistance with decreasing temperature is the $1 \mathrm{D}$ or $2 \mathrm{D}$ weak localization $[48,65]$ or a Luttinger liquid behavior $[66,123]$. 2D weak localization predicts a $\ln T$ dependence of the conductance at least when electron-phonon or electron-electron interaction are the dominating dephasing mechanism. Luttinger liquid theory, however, predicts a power law dependence. Furthermore, Luttinger theory predicts, that a large part of the resistance is physically located at the contacts to the nanotube, where the Fermi-liquid electrons must enter the collective modes of the Luttinger liquid [123, 124]. Graugnard et al. [123] interpreted their measurements as a series of the varying contact resistance due to the Luttinger liquid and a constant tube resistance. In the present work the contact resistances were measured directly and they remain nearly constant, whereas the slopes in the potential profiles that display the intrinsic resistivities vary with temperature. Consequently, for this tube Luttinger liquid behavior can be excluded at least for a temperature range above $28 \mathrm{~K}$.

The alternative mechanism for the resistance increase with temperature is weak localization. At similar tube material Stojetz et al. [54] showed with magnetoconductance measurements clear evidence for localization effects up to a temperature of $60 \mathrm{~K}$. In the past, two-dimensional [48] as well as one-dimensional [65, 66] weak localization theories were used to interpret the temperature dependence of conductance. The arguments for both dimensionalities are reasonable. The walls consist of wrapped 2D graphene sheets giving rise to the assumption that transport occurs on the 2D surface. The electron subbands, however, are one-dimensional if the phase coherence length exceeds the circumference of the tube. From magnetoconductance measurements, phase coherence lengths $L_{\varphi}$ of the order of $50 \mathrm{~nm}$ were reported for temperatures of the order of $10 \mathrm{~K}[54,68]$. For higher temperatures $L_{\varphi}$ decreases. In our accessible temperature range from room temperature down to $28 \mathrm{~K}, L_{\varphi}$ is in the range of the tube circumference. Consequently, the assumptions of $2 \mathrm{D}$ diffusive transport in the rolled graphene sheet as well as of 1D transport due to the periodic boundary conditions around the circumference are reasonable.

In two dimensions the correction to the conductivity due to weak localization without magnetic field is [108, 125]:

$$
\Delta \sigma_{2 D}=-\frac{e^{2}}{\hbar \pi^{2}} \ln \left(\frac{L_{\varphi}}{L_{m}}\right)
$$



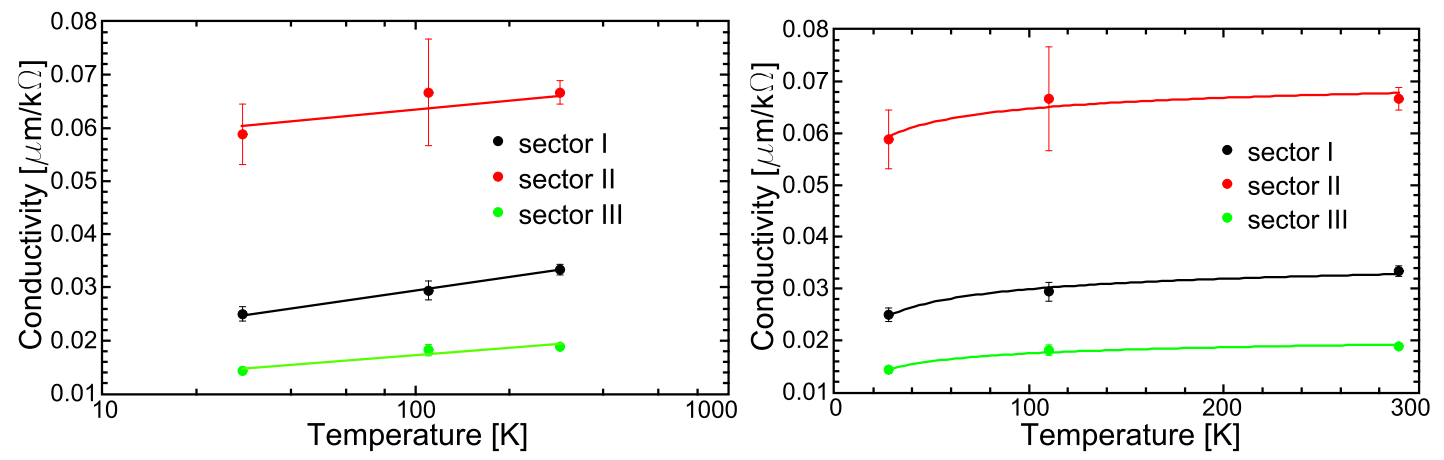

Figure 6.2: Conductivities of the three sectors as a function of the temperature (left in logarithmic scale). The solid lines in the left image represent a $\ln T$ dependence of the conductivity corresponding to $2 \mathrm{D}$ weak localization. In the right panel the measured conductivities were fitted with 1D weak localization theory.

In the one-dimensional case with width of the conductor $W$ the correction is [108]:

$$
\Delta \sigma_{1 D}=-\frac{e^{2} L_{\varphi}}{\hbar \pi W}
$$

It could be assumed that in CNTs the width $W$ is directly connected to the circumference of the tube. In contrast, in the not strictly one-dimensional CNTs (at least in multiwalled tubes) $W$ has to be treated as an independent parameter [66].

The most probable dephasing mechanism in this temperature range is Nyquist dephasing (interaction of one electron with the field of all other electrons that fluctuates due to Nyquist noise) resulting in a temperature dependence of the phase coherence length of $L_{\varphi} \sim T^{-\frac{1}{3}}$. Including this relation in the conductivity correction for $2 \mathrm{D}$ weak localization (equation (6.1)) results in a $\ln T$ dependence. In the $1 \mathrm{D}$ case, however, the conductivity correction (equation (6.2)) scales with $T^{-\frac{1}{3}}$.

In fig. 6.2 fits for both cases are presented. In the left panel the semi-log plot of the conductivity as a function of the temperature is shown. Within the error bars good fits with the $\ln T$ behavior of $2 \mathrm{D}$ weak localization could be performed. Since in fig. 6.2 the 1D conductivities are plotted the circumference of the tube is taken into account as an additional factor in equation (6.1). Therefore, the slope of sector I that exhibits the original diameter is higher than that of sectors II and III. The ratio of the slopes of 1.6 is in good correlation to the ratio of the diameters (measured by the SEM) of 1.4. Evaluating the effective width of the 2D conductor 
using the slope results e.g. for sector III in a value of $\approx 220 \mathrm{~nm}$. This is a value about five times larger than the circumference of the tube. Langer et al. [48] obtained similar results and interpreted them as multiple shells contributing to the current. At least for room temperature the present results (chapter 5) support this conclusion. For low temperatures, however, it was reported, that only one or at maximum two shells contribute to the current [68].

The right panel of fig. 6.2 shows the same conductivities fitted with $\sigma=a-b T^{-\frac{1}{3}}$ (fit parameters $a$ and $b$ ) accordant to $1 \mathrm{D}$ weak localization. It fits just as well as the $2 \mathrm{D}$ weak localization.

To draw a conclusion, the observed temperature dependence could be traced back to weak localization. But the available data of only three temperatures within one order of magnitude do not allow to distinguish between $1 \mathrm{D}$ and $2 \mathrm{D}$ weak localization. In fact, even further measurements in the accessible temperature range of only one order of magnitude, i.e. between the present temperature positions, would not be sufficient to distinguish between them as long as the conductivity cannot be determined more accurately.

\subsection{Measurements in non-local geometry}

In local geometry the linear shape of the potential profile was unchanged when switching from room temperature to low temperatures. Only the slope that represents the resistivity changed. In non-local geometry, however, the potential profiles look quite different. Also, several measurement runs do not necessarily lie on one general curve as found at room temperature ${ }^{1}$. In contrast the measured potential fluctuated partially irreproducibly.

At $T=110 \mathrm{~K}$ (fig. 6.3) the exponential behavior which was observed unambiguously at room temperature (fig. 5.15) disappeares. The potential fluctuates around a mean value of $\approx 3 \mathrm{mV}$ and exhibits a small positive slope. An irregularity occurs between $400 \mathrm{~nm}$ and $600 \mathrm{~nm}$ where a potential shift can be observed. In a thorough examination of the SEM image of the tube a slight anomaly can be noticed at this position (see SEM image in fig. 6.3).

At $T=28 \mathrm{~K}$ the potential profile looks similar with two exceptions. First, there is an additional temporary increase in the potential between $100 \mathrm{~nm}$ and $200 \mathrm{~nm}$ which cannot be attributed to an anomaly in the SEM image. Second, the area

\footnotetext{
${ }^{1}$ Although all other measurements consisted also of several individual measurement runs they were plotted in only one color since they were located at one general curve.
} 


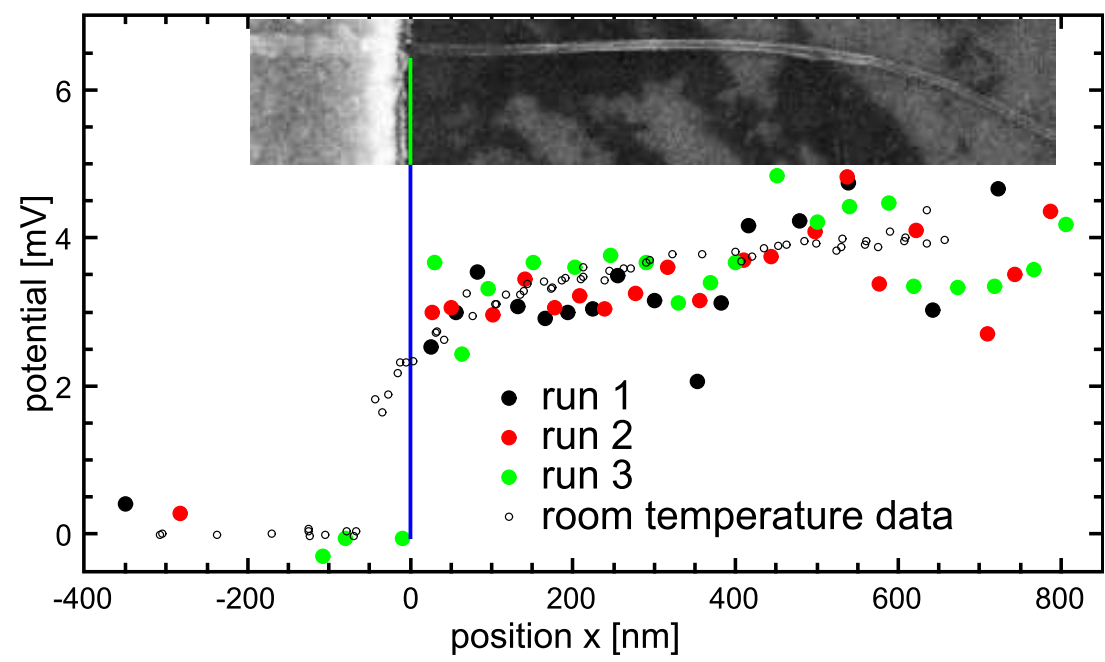

Figure 6.3: Non-local potential profile of sample D at a temperature of $T=110 \mathrm{~K}$. The clear exponential behavior at room temperature (see fig. 5.15) cannot be observed any more. In contrast the potential fluctuates with an amplitude up to $1 \mathrm{mV}$ around a nearly constant potential of $\approx 3 \mathrm{mV}$ except in the region between $400 \mathrm{~nm}$ and $600 \mathrm{~nm}$ where the potential is increased. Around this region there seems to be a small convexity in the SEM image.

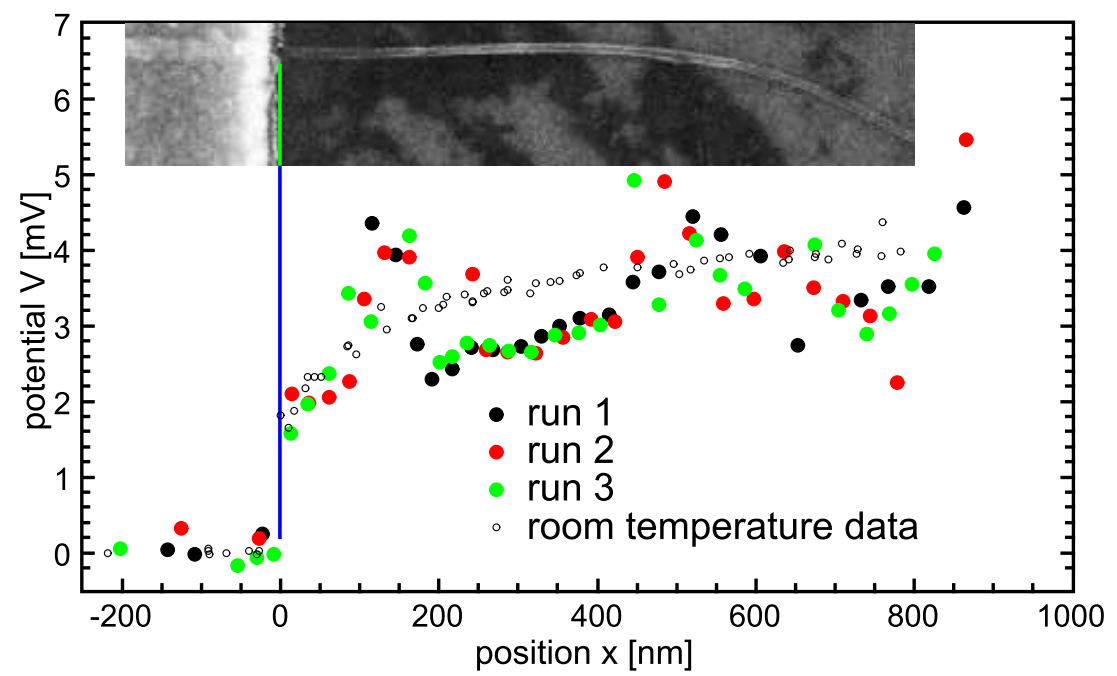

Figure 6.4: Non-local potential of sample D at a temperature of $T=28 \mathrm{~K}$. In addition to the potential increase around $500 \mathrm{~nm}$ that was already observed at $T=110 \mathrm{~K}$ there is a second region with elevated potential between $100 \mathrm{~nm}$ and $200 \mathrm{~nm}$. In the region between $200 \mathrm{~nm}$ and $400 \mathrm{~nm}$ all three measurement runs resulted in the same reproducible oscillations. 
between the two maxima was reproduced nearly exactly by all three measurement runs. In this region the increasing trend is superimposed by a small reproducible oscillation.

These three effects, namely the irreproducible fluctuations at a small scale (best seen in fig. 6.3), the conspicuous local increase of the potential and the reproducible oscillating potential at $28 \mathrm{~K}$ cannot be explained classically. In fact, even in the quantum transport regime an inconstant non-local potential is only possible if nonzero transmission probabilities to both contacts exist (see equation (4.2)), i.e. even to the electrode farther away. Two pathways to this electrode are imaginable. First, the straight way passing the second contact in the outermost shell directly below the electrode. Although Ke et al. [126] calculated that the transmission straight through the contact is drastically decreasing even for narrow contacts, Makarovski et al. [127] and Gunnarsson et al. [128] both found a non-constant non-local potential in SWCNTs indicating a non-vanishing transmission through the contact. For the MWCNT used in our experiment, an additional pathway that does not cross the contact is possible. Similar to the room temperature behavior the electrons can exit the outermost shell, pass the second contact in an undisturbed lower shell and reenter the outermost shell again beyond the electrodes.

The irreproducible fluctuations at a small scale for example between 0 and $400 \mathrm{~nm}$ at $110 \mathrm{~K}$ can be attributed to the limited accuracy of the tip positioning combined with interference effects. The digital steps alone aggregate an uncertainty of $\approx 4 \mathrm{~nm}$. An additional error, which is even more pronounced at low temperatures, results from the thermal drift that was corrected only with a drift function linear in time. Furthermore, only the position along the tube axis was recorded. Since the MWCNT is not strictly one dimensional a variation perpendicular to the tube is also possible. All described effects combine to a uncertainty in the position of about $10 \mathrm{~nm}$. Consequently small scale effects cannot be recorded with the present technique but can give rise to fluctuations that seems to be irreproducible at first glance.

One possible reason for small scale variations in the potential are induced by Friedel oscillations. They arise from the electron waves in the conductor being reflected at impurities phase coherently. The incoming and reflected waves interfere and form standing waves whose anti-nodes (nodes) at position $x$ increase (decrease) the local density of states at point $x$. Since both contacts inject electron waves that can be handled independently in the phase coherent state, two partial local densities of states exist that are equal to the injectivites of the according contacts [105]. The oscillations in the injectivities have wave lengths of 
$\lambda=\frac{2 \pi}{k_{F}} \approx 4 \AA$ whereby $\mathbf{k}_{\mathbf{F}}$ denotes the Fermi wavevector. Since the measured potential is the average of the potentials of the electrodes, weighted with the injectivities (see equation (4.3)) the resulting potential has also a periodicity in the range of $4 \AA$. This is well below the lateral resolution that results in apparently random oscillations depending on the exact position of the tip on the tube.

Another approach for these fluctuations is given by Makarovski et al. [127] and Gunnarsson et al. [128]. The authors found significant voltages in four-point measurements of SWCNTs in non-local geometry and traced it back to different coupling of the probes to the two orbital modes of the tube. Due to the different interaction of the two modes with the evaporated contacts, the transmission probabilities can be modulated differently and consequently their electrochemical potentials (as defined in equation (4.3)) differ from each other.

In equation (4.3) it was stated that the measured potential is independent of the coupling strength. This results from the square of the coupling energy contributing linearly to all $T_{\alpha \beta}$ (equation (1) of ref. [105]). In the case of two independently contributing modes, however, the two different coupling constants cannot cancel. The measured potential depends on the coupling energies $t$ and $\tilde{t}$ to the two modes as follows ( distinguishes the two modes.):

$$
\mu_{3}=\frac{\left(|t|^{2} \nu_{x 1}+|\tilde{t}|^{2} \tilde{\nu}_{x 1}\right) \mu_{1}+\left(|t|^{2} \nu_{x 2}+|\tilde{t}|^{2} \tilde{\nu}_{x 2}\right) \mu_{2}}{|t|^{2} \nu(x)+|\tilde{t}|^{2} \tilde{\nu}(x)}
$$

The measured potential $\mu_{3}$ lies between the two potentials that could be measured if the probe couples only to one mode.

In the present case of a movable nanocontact the coupling strengths to the two modes can vary and consequently the measured potential deviate from each other. Due to the limited accuracy in the positioning even if one tries to measure at the same position the coupling constants to the two modes can vary and consequently the potential profile seems to be irreproducible.

Both discussed reasons for the potential fluctuation, the Friedel-like oscillations as well as the differing coupling strengths to the two orbital modes result in principle in well defined potential measurement values ${ }^{1}$ but occur at such scales that they are not accessible with the available technique. Thus these effects cannot be distinguished with the available data.

\footnotetext{
${ }^{1}$ The measuring error extracted from single spectroscopy curves is well below the fluctuation height of $\approx 1 \mathrm{mV}$.
} 
The main effect, in the regions with locally increased potential, occurs at a considerably larger scale. Since at least at the region at about $500 \mathrm{~nm}$ the structure of the nanotube exhibits a small structural defect a connection seems to be evident. Obviously this defect does not modify the transport properties considerably at room temperature. Non-local room temperature curves, recorded after the low temperature measurements, show the same exponential behavior as above mentioned (fig. 5.15) and therefore the differences cannot be attributed to a structural damage that occurred during the low temperature measurements. The room temperature measurements show also that the outer shells cannot be disrupted since this would lead to a horizontal asymptote (see potential profile of sample B, fig. 5.6). In classical terms it is not clear why the measured potential decreases again after the defect to the value before the defect. So, this effect can be only discussed in terms of quantum conductance.

For low temperature it was reported that only outer shells contribute to the transport properties [68], consequently the intershell conductance has to be much lower than determined for room temperature. A lower intershell coupling, however, gives rise to the assumption that the electrochemical potentials in the outermost and the second shell are considerably different. If the coupling between the STM tip and the outermost shell of the tube is reduced due to structural defects the feedback control reduces the tip-nanotube distance in order to keep a constant tunnel current. The direct coupling between tip and second shell is typically negligible due to the higher distance to the tip and the exponential behavior of the tunnel current. With a decreased distance to the tip and a reduced coupling between outermost shell and tip the contribution of the second shell also has to be considered. So the second shell can contribute significantly to the current in the tip resulting in an increased potential.

At the second position where this effect occurs at the lower temperature of $28 \mathrm{~K}$ no structural defects at all can be observed within the resolution of the SEM image. Possible reasons for a lower coupling to the outermost shell might be some doping impurities that deplete the local density of states as well as adsorbates on the surface.

The last remarkable effect of the non-local low temperature measurements is the reproducible oscillating potential in the area ranging from $200 \mathrm{~nm}$ to $400 \mathrm{~nm}$ at $T=28 \mathrm{~K}$. The question why irreproducible fluctuations are strongly suppressed in this area is unsolved so far. Only one outlier in this region could be observed at $x=240 \mathrm{~nm}$. All other values lie close to one general oscillating curve. The explanation for this oscillating potential is, similar to the universal conductance 


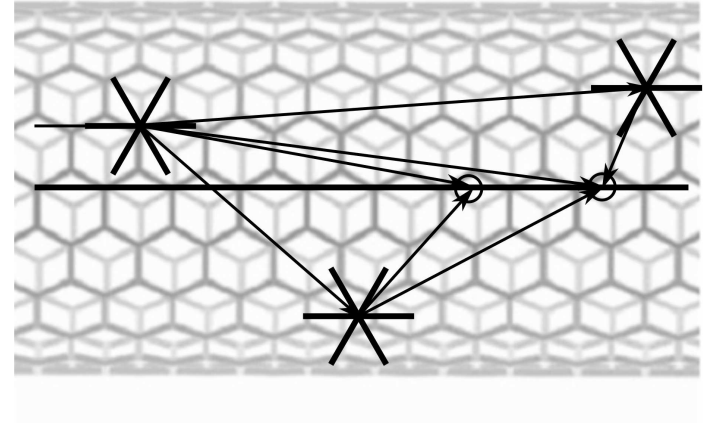

Figure 6.5: Several paths of one electron that hits the left scattering center. At every measurement position (two are illustrated, marked with circles) the injectivity to a probe (tip) is represented by the interference of all possible pathways.

fluctuations, a result of interference of several paths that included phase coherent scattering centers. The illustration in fig. 6.5 shows possible pathways after an electron is scattered at the leftmost scattering center. At the measurement position all amplitudes of the paths that depend on the scattering cross sections sum up with appropriate phase relation. Electrons originating from different contact electrodes form a specific interference pattern for each contact whose intensities represents the injectivities at this position. The resulting measured potential can be calculated as in equation 4.3. Depending on the density of scattering centers and on the position of the scattering centers the potential profile exhibit irregular but reproducible oscillations as observed.

In summary, the low temperature potential profile of sample D was measured in local as well as in non-local geometry. The average resistivity increases with decreasing temperature. The resistance increase can be interpreted in terms of weak localization but within the available temperature range and precision it cannot be distinguished between 1D od 2D weak localization.

In the non-local data, however, the exponential behavior that indicates classical diffusive intershell transport disappears at low temperature. Instead of that several quantum conductance effects appear. Fluctuations that appear as irreproducible are a result of small scale potential variations combined with the uncertainty in the lateral position of the probe. Furthermore, regions with strongly increased potential can be attributed to a reduced coupling to the outermost shell and therefore the transmission probability in the second shell can contribute considerably. In a region at $28 \mathrm{~K}$ where no irreproducible fluctuations occurred an oscillating behavior was observed that can be interpreted in terms of interference of several electron paths similar to universal conductance fluctuations. 


\section{Summary}

In this thesis electronic transport properties of multiwall carbon nanotubes have been examined. In particular, the influence of the multiple shell geometry and the impact of structural defects on the conductance were investigated.

In order to gain access to these material parameters experimentally, MWCNTs were placed on a thin insulator surface covering a conducting layer. Metal electrodes with low contact resistance have been evaporated on top as source and drain. The tip of a scanning tunneling microscope was used to localize the tube and to act as a movable voltage probe. For this purpose the spectroscopy mode was used since it allows extracting the potential in a voltage compensated state. Due to the tunneling contact and the zero current flow the intrinsic properties are not influenced by the voltage probe.

With this experimental setup the potential profile of the outermost shell or the electrode where the tube is covered by metal was recorded as a function of the position while a constant current was flowing through the tube. The contact resistance can be directly read out from the potential step at the metal tube transition. The measurements were performed in a local as well as in a non-local geometry at room temperature and at low temperatures.

The room temperature data were interpreted in terms of classical diffusive transport using a resistance model. The present model incorporates two shells where the second represents the effective parameters of the inner tube. It considers the finite dimensions of the contacts and the finite total length of the tube. The model rebuilds well the measured potential profile of a tube without obvious defects and yields additional information on the current paths over the shells and the current density in the injection zone. Deviations between the model and the measured data could be traced back to asymmetrically evaporated contacts. One main result of this work is a considerably higher conductance between the shells at room temperature than published so far [94]. This difference was traced back to a large impact of the array of contacts on the tube of the previous experiments.

The resistance model could also be fitted well to the potential profile of a MWCNT 
with an incomplete outermost shell. The extracted parameters like intershell conductivity and intrashell resistivity are of the same order of magnitude than those of a tube without obvious defect. Furthermore the potential profile of a tube with an intramolecular junction exhibited an anomaly including a change of slope that was directly attributed to the position where the diameter changes, indicating a transition from a metallic to a semiconducting tube. The local potential as a function of the gate voltage does not reveal any significant gate dependency at room temperature.

One sample, originally without obvious defect, was stretched and kinked by means of applying a force perpendicular to the tube with the STM tip. After manipulation, the structure exhibited three regions with individual geometrical properties. The differences of these regions are manifested in the potential profile with distinct slopes which represent different resistivities. Generally, the resistivities of all regions were increased during manipulation. The sector of the tube protruding the contacts was not manipulated and exhibits an exponentially decreasing potential as the model predicted. The extracted tube parameters are similar to those of the previous samples.

Further experiments on the same sample at low temperatures revealed several temperature dependent effects. The potential profile in local geometry reveals an increasing resistivity with decreasing temperature. It can be attributed to the weak localization theory and therefore indicates a decreasing amount of phase destroying inelastic scattering.

The data recorded in non-local geometry showed several anomalies that have to be described with quantum interference effects. In particular, it can be stated that not only the position of the probe but also the ratio of the coupling strengths to several modes like the orbital modes of one tube or modes of lower shells affect the measured potential. Furthermore, oscillations in the potential profile can be attributed to the interference of several pathways that arise from scattering at inhomogeneously distributed scattering centers. 


\section{Bibliography}

[1] H. P. Boehm, R. Setton, and E. Stumpp. Nomenclature and terminology of graphite intercalation compounds. Pure \& Appl. Chem. 66: 1893 (1994).

[2] C. Frondel and U. B. Marvin. Lonsdaleite, a hexagonal polymorph of diamond. Nature 214: 587 (1967).

[3] H. W. Kroto, J. R. Heath, S. C. O'Brien, R. F. Curl, and R. E. Smalley. $C_{60}$ : Buckminsterfullerene. Nature 318: 162 (1985).

[4] W. Krätschmer, L. D. Lamb, K. Forstiropoulos, and D. R. Huffman. Solid $c_{60}$ : a new form of carbon. Nature 347: 354 (1990).

[5] S. Iijima. Direct observation of the tetrahedral bonding in graphitized carbon black by high resolution electron microscopy. J. Cryst. Growth 50: 675 (1980).

[6] S. Iijima. Helical microtubules of graphitic carbon. Nature 354: 56 (1991).

[7] S. Iijima and T. Ichihashi. Single-shell carbon nanotubes of 1-nm diameter. Nature 636: 603 (1993).

[8] D. S. Bethune, C. H. Klang, M. S. de Vries, G. Gorman, R. Savoy, J. Vazquez, and R. Beyers. Single-shell carbon nanotubes of 1-nm diameter. Nature 636: 603 (1993).

[9] A. Thess, R. Lee, P. Nikolaev, H. Dai, P. Petit, J. Robert, C. Xu, Y. H. Lee, S. G. Kim, A. G. Rinzler, D. T. Colbert, G. E. Scuseria, D. Tománek, J. E. Fischer, and R. E. Smalley. Crystalline ropes of metallic carbon nanotubes. Science 273: 483 (1996).

[10] H. Dai, A. G. Rinzler, P. Nikolaev, A. Thess, D. T. Colbert, and R. E. Smalley. Single-wall nanotubes produced by metal-catalyzed disproportionation of carbon monoxide. Chem. Phys. Lett. 260: 471 (1996). 
[11] L. D. Landau. Zur theorie der phasenumwandlungen ii. Phys. Z. Sowjetunion 11: 26 (1937).

[12] K. S. Novoselov, A. K. Geim, S. V. Morozov, D. Jiang, Y. Zhang, S. V. Dubonos, I. V. Grigorieva, and A. A. Firsov. Electric field effect in atomically thin carbon films. Science 306: 666 (2004).

[13] P. R. Wallace. The band theory of graphite. Phys. Rev. 71: 622 (1946).

[14] K. S. Novoselov, A. K. Geim, S. V. Morozov, D. Jiang, M. I. Katsnelson, I. V. Girgorieva, S. V. Dubonos, and A. A. Firsov. Two-dimensional gas of massless dirac fermions in graphene. Nature 438: 197 (2005).

[15] Y. Zhang, Y. W. Tan, H. L. Stormer, , and P. Kim. Experimental observation of the quantum hall effect and berry's phase in graphene. Nature 438: 201 (2005).

[16] L. V. Radushkevich and V. M. Lukyanovich. O strukture ugleroda, obrazujucegosja pri termiceskom razlozenii okisi ugleroda na zeleznom kontakte. Zurn Fisic Chim 26: 88 (1952).

[17] From: User mstroeck on en.wikipedia; licence: Gfdl.

[18] T. Hayashi, Y. A. Kim, T. Matoba, M. Esaka, K. Nishimura, T. Tsukada, M. Endo, and M. S. Dresselhaus. Smallest freestanding single-walled carbon nanotube. Nano Letters 3: 887 (2003).

[19] S. Lebedkin, P. Schweissb, B. Renkerb, S. Malika, F. Hennrichc, M. Neumaierc, C. Stoermerc, and M. M. Kappesa. Single-wall carbon nanotubes with diameters approaching $6 \mathrm{~nm}$ obtained by laser vaporization. Carbon 40: 417 (2002).

[20] C. H. Kiang. Growth of large-diameter single-walled carbon nanotubes. J. Phys. Chem. A 104: 2454 (2000).

[21] J. A. Elliott, J. K. W. Sandler, A. H. Windle, R. J. Young, , and M. S. P. Shaffer. Collapse of single-wall carbon nanotubes is diameter dependent. Phys. Rev. Lett. 92: 095501 (2004).

[22] L. Henrard, A. Loiseau, C. Journet, and P. Bernier. Study of the symmetry of single-wall nanotubes by electron diffraction. Eur. Phys. J. B 13: 661 (2004). 
[23] P. X. Houa, C. Liua, and H. M. Cheng. Purification of carbon nanotubes. Carbon 46: 661 (2008).

[24] J. C. Charlier, X. Blase, and S. Roche. Electronic and transport properties of nanotubes. Rev. Mod. Phys. 79: 677 (2007).

[25] J. C. Charlier. Defects in carbon nanotubes. Acc. Chem. Res. 35: 1063 (2002).

[26] A. J. Stone and D. J. Wales. Theoretical studies of icosahedral $c_{60}$ and some related species. Chem. Phys. Lett. 128: 501 (1986).

[27] P. Lambin, A. Fonseca, J. P. Vigneron, J. B. Nagy, and A. A. Lucas. Structural and electronic properties of bent carbon nanotubes. Chem. Phys. Lett. 245: 85 (1995).

[28] M. Liuand and J. M. Cowley. Structures of carbon nanotubes studied by hrtem and nanodiffraction. Ultramicroscopy 53: 333 (1994).

[29] J. G. Lavina, S. Subramoney, R. S. Ruoffb, S. Berberc, and D. Tománek. Scrolls and nested tubes in multiwall carbon nanotubes. Carbon 40: 1123 (2002).

[30] P. J. F. Harris. Carbon Nanotube Science (Cambridge university press, 2009).

[31] S. Agrawal, M. S. Raghuveer, H. Li, and G. Ramanath. Defect-induced electrical conductivity increase in individual multiwalled carbon nanotubes. Appl. Phys. Lett. 90: 193104 (2007).

[32] P. Jensen, J. Gale, , and X. Blase. Catalysis of nanotube plasticity under tensile strain. Phys. Rev. B 66: 193403 (2002).

[33] D. Orlikowski, M. B. Nardelli, J. Bernholc, and C. Roland. Theoretical stm signatures and transport properties of native defects in carbon nanotubes. Phys. Rev. B 61: 14194 (2000).

[34] M. B. Nardelli, B. I. Yakobson, and J. Bernholc. Brittle and ductile behavior in carbon nanotubes. Phys. Rev. Lett. 81: 4656 (1998).

[35] T. Belytschko, S. P. Xiao, G. C. Schatz, and R. S. Ruoff. Atomistic simulations of nanotube fracture. Phys. Rev. B 65: 235430 (2002). 
[36] D. H. Kim and K. J. Chang. Electron transport in telescoping carbon nanotubes. Phys. Rev. B 66: 155402 (2002).

[37] J. Cumings and A. Zettl. Localisation and nonlinear resistance in telescopically extended nanotubes. Phys. Rev. Lett. 93: 086801 (2004).

[38] P. Poncharal, Z. L. Wang, D. Ugarte, and W. A. de Heer. Electrostatic deflections and electromechanical resonances of carbon nanotubes. Science 238: 1513 (1999).

[39] S. Iijima, C. Brabec, A. Maiti, and J. Bernholc. Structural flexibility of carbon nanotubes. J. Chem. Phys. 104: 2089 (1996).

[40] A. Loiseau, P. Launois, P. Petit, S. Roche, and J.-P. Salvetate. Understanding Carbon Nanotubes From Basics to Applications (Springer-Verlag Berlin, 2006).

[41] J. C. Charlier, X. Gonze, and J. P. Michenaud. First-principles study of the electronic properties of graphite. Phys. Rev. B 43: 4579 (1991).

[42] C. Bena and G. Montambaux. Remarks on the tight-binding model of graphene. New Journal of Physics 11: 095003 (2009).

[43] L. V. Hove. The occurrence of singularities in the elastic frequency distribution of a crystal. Phys. Rev. 89: 1189 (1952).

[44] C. L. Kane and E. J. Mele. Size, shape, and low energy electronic structure of carbon nanotubes. Phys. Rev. Lett 78: 1932 (1997).

[45] X. Blase, L. X. Benedict, E. L. Shirley, and S. G. Louie. Hybridization effects and metallicity in small radius carbon nanotubes. Phys. Rev. Lett. 72: 1878 (1993).

[46] M. Ouyang, J.-L. Huang, C. L. Cheung, and C. M. Lieber. Energy gaps in 'metallic' single-walled carbon nanotubes. Science 292: 702 (2001).

[47] P. Delaney, H. J. Choi, J. Ihm, S. G. Louie, and M. L. Cohen. Broken symmetry and pseudogaps in ropes of carbon nanotubes. Nature 391: 466 (1998).

[48] L. Langer, V. Bayot, E. Grivei, J. P. Issi, J. P. Heremans, C. H. Olk, L. Stockman, C. V. Haesendonck, and Y. Bruynseraede. Quantum transport in a multiwalled carbon nanotube. Phys Rev. Lett. 76: 479 (1996). 
[49] L. Langer, L. Stockman, J. P. Heremans, V. Bayot, C. Olk, C. V. Haesendonck, Y. Bruynseraede, and J.-P. Issi. Electrical resistance of a carbon nanotube bundle. J. Mater. Res. 9: 927 (1994).

[50] S. N. Song, X. K. Wang, R. P. H. Chang, and J. B. Ketterson. Electronic properties of graphite nanotubules from galvanomagnetic effects. Phys. Rev. Lett. 72: 697 (1994).

[51] R. Saito, G. Dresselhaus, and M. S. Dresselhaus. Physical properties of carbon nanotubes (Imperial College Press, 1998).

[52] F. Triozon, S. Roche, A. Rubio, and D. Mayou. Electrical transport icarbon nanotubes: Role of disorder and helical symmetries. Phys. Rev. B 69: 121410 (2004).

[53] S. Wang, M. Grifoni, and S. Roche. Anomalous diffusion and elastic mean free path in disorder-free multiwalled carbon nanotubes. Phys. Rev. B 74: 121407 (2006).

[54] B. Stojetz, C. Miko, L. Forró, and C. Strunk. Effect of band structure on quantum interference in multiwall carbon nanotubes. Phys. Rev. Lett. 94: $186802(2005)$.

[55] S. Frank, P. Poncharal, Z. L. Wang, and W. A. de Heer. Carbon nanotube quantum resistors. Science 280: 1744 (1998).

[56] A. Urbina, I. Echeverría, A. Pérez-Garrido, A. Díaz-Sánchez, and J. Abellán. Quantum conductance steps in solutions of multiwalled carbon nanotubes. Phys. Rev. Lett. 90: 106603 (2003).

[57] H. J. Li, W. G. Lu, J. J. Li, X. D. Bai, and C. Z. Gu. Multichannel ballistic transport in multiwalled carbon nanotubes. Phys. Rev. Lett. 95: 086601 (2005).

[58] C. Berger, Y. Yi, Z. L. Wang, and W. A. de Heer. Multiwalled carbon nanotubes are ballistic conductors at room temperature. Appl. Phys. A 74: 363 (2002).

[59] E. Brown, L. Hao, J. C. Gallop, and J. C. Macfarlane. Ballistic thermal an electrical conductance measurements on individual multiwall carbon nanotubes. Appl. Phys. Lett. 87: 023107 (2005). 
[60] A. Bachtold, M. S. Fuhrer, S. Plyasunov, M. Forero, E. H. Anderson, A. Zettl, and P. L. McEuen. Scanned probe microscopy of electronic transport in carbon nanotubes. Phys. Rev. Lett. 84: 6082 (2000).

[61] P. J. Pablo, C. G. Navarro, J. Colchero, P. A. Serena, J. G. Herrero, and A. M. Baró. Nonlinear resistance versus length in singe-walled carbon nanotubes. Phys. Rev. Lett. 88: 036804 (2002).

[62] Y. Yaish, J. Y. Park, S. Rosenblatt, V. Sazonova, M. Brink, and P. L. McEuen. Electrical nanoprobing of semiconducting carbon nanotubes using an atomic force microscope. Phys. Rev. Lett. 92: 046401 (2004).

[63] R. Hobara, S. Yoshimoto, T. Ikuno, M. Katayama, N. Yamauchi, W. Wongwiriyapan, S. Honda, I. Matsuda, S. Hasegawa, and K. Oura. Electronic transport in multiwalled carbon nanotubes contacted with patterned electrodes. Phys. Rev. Lett. 43: 1081 (2004).

[64] A. Stetter, J. Vancea, and C. H. Back. Determination of the intershell conductance in a multiwall carbon nanotube. Appl. Phys. Lett. 93: 172103 (2008).

[65] K. Liu, P. Avouris, and R. Martel. Electrical transport in doped multiwalled carbon nanotubes. Phys. Rev. B 63: 161404 (2001).

[66] C. Schönenberger, A. Bachtold, C. Strunk, J. P. Salvetat, and L. Forró. Interference and interaction in multi-wall carbon nanotubes. Appl. Phys. A 69: 283 (1999).

[67] B. L. Al'tshuler, A. G. Aronov, and B. Z. Spivak. The aaronov-bohm effect in disordered conductors. JETP Lett. 33: 94 (1981).

[68] A. Bachtold, C. Strunk, J. Salvetat, J. Bonard, L. Forró, T. Nussbaumer, and C. Schönenberger. Aharonov-bohm oscillations in carbon nanotubes. Nature 397: 673 (1999).

[69] S. J. Tans, M. H. Devoret, H. Dai, A. Thess, R. E. Smalley, L. J. Geerligs, and C. Dekker. Anomalous diffusion and elastic mean free path in disorderfree multiwalled carbon nanotubes. Nature 386: 474 (1997).

[70] M. Bockrath, D. H. Cobden, P. L. McEuen, N. G. Chopra, A. Zettl, A. Thess, and R. E. Smalley. Single-electron transport in ropes of carbon nanotubes. Science 575: 1922 (1997). 
[71] A. Bezryadin, A. R. M. Verschueren, S. J. Tans, and C. Dekker. Multiprobe transport on individual single-wall carbon nanotubes. Phys. Rev. Lett. 80: 4036 (1998).

[72] B. Gao, Y. F. Chen, M. S. Fuhrer, D. C. Glattli, and A. Bachtold. Fourpoint resistance of individual single-wall carbon nanotubes. Phys. Rev. Lett. 95: 196802 (2005).

[73] M. Bockrath, D. H. Cobden, J. L. nd A. G. Rinzler, R. E. Smalley, L. Balents, and P. L. McEuen. Luttinger-liquid behaviour in carbon nanotubes. Nature 397: 598 (1999).

[74] J. Appenzeller, J. Knoch, V. Derycke, R. Martel, S. Wind, , and P. Avouris. Field-modulated carrier transport in carbon nanotube transistors. Phys. Rev. Lett. 89: 126801 (2002).

[75] V. Derycke, R. Martel, J. Appenzeller, and P. Avouris. Controlling doping and carrier injection in carbon nanotube transistors. Appl. Phys. Lett. 80: 2773 (2002).

[76] M. S. Purewal, B. H. Hong, A. Ravi, B. Chandra, J. Hone, and P. Kim. Scaling of resistance and electron mean free path of single-walled carbon nanotubes. Phys. Rev. Lett 98: 186808 (2007).

[77] J. Park, S. Rosenblatt, Y. Yaish, V. Sazonova, H. Üstünel, S. Braig, T. A. Arias, P. W. Brouwer, and P. L. McEuen. Electron-phonon scattering in metallic single walled carbon nanotubes. Nano Letters 4: 517 (2004).

[78] X. Zhou, J.-Y. Park, S. Huang, J. Liu, and P. L. McEuen. Band structure, phonon scattering, and the performance limit of single-walled carbon nanotube transistors. Phys. Rev. Lett 95: 146805 (2005).

[79] M. Freitag, A. T. Johnson, S. V. Kalinin, and D. A. Bonnell. Role of defects in electronic transport through carbon nanotube field-effect transistors. Phys. Rev. Lett. 89: 216801 (2002).

[80] S. Wang and M. Grifoni. Helicity and electron-correlation effects on transport properties of double-walled carbon nanotubes. Phys. Rev. Lett. 95: $266802(2005)$.

[81] A. Rochefort, P. Avouris, F. Lesage, and D. R. Salahub. Electrical and mechanical properties of distorted carbon nanotubes. Phys. Rev. B 60: 13824 (1999). 
[82] M. B. Nardelli and J. Bernholc. Mechanical deformations and coherent transport in carbon nanotubes. Phys. Rev. B 60: 16338 (1999).

[83] M. B. Nardelli, J. L. Fattebert, D. Orlikowski, C. Roland, Q. Zhao, and J. Bernholc. Mechanical properties, defects and electronic behavior of carbon nanotubes. Carbon 38: 1703 (2000).

[84] T. Kuzumaki and Y. Mitsuda. Dynamic measurement of electrical conductivity of carbon nanotubes during machanical deformation by nanoprobe manipulation in transmission electron microscopy. Appl. Phys. Lett 85: 1250 (2004).

[85] H. S. Jang, Y. H. Lee, H. J. Na, and S. H. Nahm. Variation in electrical resistance versus strain of an individual multiwalled carbon nanotube. J. App. Phys. 104: 114304 (2008).

[86] T. W. Tombler, C. Zhou, L. Alexeyev, J. Kong, H. Dai, L. Liu, C. S. Jayanthi, M. Tang, and S. Y. Wu. Reversible electromechanical characteristics of carbon nanotubes under local-probe manipulation. Nature 405: 769 (2000).

[87] D. Bozovic, M. Bocjrath, J. H. Hafner, C. M. Lieber, and H. Park. Plastic deformations in mechanically strained single-walled carbon nanotubes. Phys. Rev. B 67: 33407 (2003).

[88] S. Sanvito, Y.-K. Kwon, D. Tománek, and C. J. Lambertk. Fractional quantum conductance in carbon nanotubes. Phys. Rev. Lett. 84: 1974 (2000).

[89] S. Roche, F. Triozon, A. Rubio, and D. Mayou. Conduction mechanisms and magnetotransport in multiwalled carbon nanotubes. Phys. Rev. B 64: 121401 (2001).

[90] Y.-G. Yoon, P. Delaney, and S. G. Louie. Quantum conductance of multiwall carbon nanotubes. Phys. Rev. B 66: 073407 (2002).

[91] A. Hansson and S. Stafström. Intershell conductance in multiwall carbon nanotubes. Phys. Rev. B 67: 075406 (2003).

[92] A. M. Lunde, K. Flensberg, and A. P. Jauho. Intershell resistance in multiwall carbon nanotubes: a coulomb drag study. Phys. Rev. B 71: 125408 (2005).

[93] P. G. Collins, M. S. Arnold, and P. Avouris. Engineering carbon nanotubes and nanotube circuits using electrical breaktown. Science 292: 706 (2001). 
[94] B. Bourlon, C. Miko, L. Forró, D. C. Glattli, and A. Bachtold. Determination of the intershell conductance in multiwalled carbon nanotubes. Phys. Rev. Lett. 93: 176806 (2004).

[95] F. W. K. Katayamaa and K. Gamoa. Contact resistance of multiwall carbon nanotubes. Microelectron. Eng. 67: 853 (2003).

[96] D. Mann, A. Javey, J. Kong, Q. Wang, and H. Dai. Ballistic transport in metallic nanotubes with reliable pd ohmic contacts. Nano Lett. 3: 1541 (2003).

[97] H. Dai, E. W. Wong, and C. M. Lieber. Probing electrical transport in nanomaterials: conductivity of individual carbon nanotubes. Science 272: 523 (1996).

[98] W. F. Brinkman and R. C. Dynes. Tunneling conductance of asymmetrical barriers. J. App. Phys. 41: 1915 (1970).

[99] A. Stetter. Leitfähigkeitsmessungen an kohlenstoffnanorörchen mit beweglichen nanokontakten. Diplomarbeit, Universität Regensburg (2006).

[100] A. J. Babić, J. Furer, M. Iqbal, and C. Schönenberger. Suitability of carbon nanotubes grown by chemical vapour deposition for electrical devices. AIPProceedings 723: 574 (2004).

[101] A. Javey, J. Guo, Q. Wang, M. Lundstrom, and H. Dai. Ballistic carbon nanotube field-effect transistors. Nature 424: 654 (2003).

[102] Z. Chen, J. Appenzeller, J. Knoch, Y. Lin, and P. Avouris. The role of metal-nanotube contact in the performance of carbon nanotube field-effect transistors. Nano Lett. 5: 1497 (2005).

[103] N. Nemec, D. Tománek, and G. Cuniberti. Contact dependence of carrier injection in carbon nanotubes: an an initio study. Phys. Rev. Lett. 96: 076802 (2007).

[104] Discussion with prof. jascha repp.

[105] T. Gramespacher and M. Büttiker. Nanoscopic tunneling contacts on mesoscopic multiprobe conductors. Phys. Rev. B 56: 13026 (1997).

[106] M. Büttiker. Four-terminal phase coherent resistance. Phys. Rev. Lett 57: 1761 (1986). 
[107] M. Büttiker. Symmetry of electrical conduction. IBM J. Res. Dev. 32: 317 (1988).

[108] S. Datta. Electronic transport in mesoscopic systems (Cambridge university press, 2007), 7. edition.

[109] K. Shabrawy, K. Maharatna, D. M. Bagnall, and B. M. Al-Hashimi. Modeling swcnt band-gap and effective mass variation using a monte carlo approach. IEEE Trans. Nano. (2009).

[110] J. M. Cowley and S. D. Packard. Coherent nanodiffraction from phase objects: carbon nanotubes. Ultramicroscopy 63: 39 (1996).

[111] C. H. Kiang, M. Endo, P. M. Ajayan, G. Dresselhaus, and M. S. Dresselhaus. Size effects in carbon nanotubes. Phys. Rev. Lett. 81: 1869 (1998).

[112] W. Primak and L. H. Fuchs. Electrical conductivities of natural graphite crystals. Phys. Rev. 95: 22 (1954).

[113] K. Matsubara, K. Sugihara, and T. Tsuzuku. Electrical resistance in the $c$ direction of graphite. Phys. Rev. B 41: 969 (1990).

[114] H. Schmidt, T. Lüdtke, P. Barthold, and R. J. Haug. Mobilities and scattering times in decoupled graphene monolayers. Phys. Rev. B 81: 121403 (2010).

[115] L. W. Nagel and D. Pederson. Spice (simulation program with integrated circuit emphasis). Technical Report UCB/ERL M382, EECS Department, University of California, Berkeley (1973).

[116] Z. Yao, H. C. Postma, L. Balents, and C. Dekker. Carbon nanotube intramolecular junctions. Nature 402: 273 (1999).

[117] S. Paulson, M. R. Falvo, N. Snider, A. Helser, T. Hudson, A. Seeger, R. M. Taylor, R. Superfine, and S. Washburn. In situ resistance measurements of strained carbon nanotubes. Appl. Phys. Lett. 75: 2936 (1999).

[118] M. BuongiornoNardelli, B. I. Yakobson, and J. Bernholc. Mechanism of strain release in carbon nanotubes. Phys. Rev. B 57: R4277 (1998).

[119] M. J. Biercuk, N. Mason, J. M. Chow, and C. M. Marcus. Locally addressable tunnel barriers within a carbon nanotube. Nano Lett. 4: 2499 (2004). 
[120] D. Bozovic, M. Bockrath, J. H. Hafner, C. M. Lieber, H. Park, and M. Tinkham. Electronic properties of mechanically induced kinks in single-walled carbon nanotubes. Appl. Phys. Lett. 78: 3693 (2001).

[121] J. Cao, Q. Wang, and H. Dai. Electromechanical properties of metallic, quasimetallic, and semiconducting carbon nanotubes under stretching. Phys. Rev. Lett. 90: 157601 (2003).

[122] L. Yang and J. Han. Electronic structure of deformed carbon nanotubes. Phys. Rev. Lett. 85: 154 (2000).

[123] E. Graugnard, P. J. de Pablo, B. Walsh, A. W. Ghosh, S. Datta, and R. Reifenberger. Temperature dependence of the conductance of multiwalled carbon nanotubes. Phys. Rev. B 64: 125407 (2001).

[124] C. L. Kane and M. P. A. Fisher. Transmission through barriers and resonant tunneling in an interacting one-dimensional electron gas. Phys. Rev. B 46: 15233 (1992).

[125] P. A. Lee and T. V. Ramakrishnan. Disordered electronic systems. Rev. Mod. Phys. 57: 287 (1985).

[126] S. H. Ke, W. Yang, and H. U. Baranger. Nanotube-metal junctions: 2- and 3-terminal electrical transport. J. Chem. Phys. 124: 124 (2006).

[127] A. Makarovski, A. Zhukov, J. Liu, and G. Finkelstein. Four-probe measurements of carbon nanotubes with narrow metal contacts. Phys. Rev. B 76: 161405 (2007).

[128] G. Gunnarsson, J. Trbovic, and C. Schönenberger. Large oscillating nonlocal voltage in multiterminal single-wall carbon nanotube devices. Phys. Rev. B 77: 201405 (2008). 



\section{Acknowledgement - Danksagung}

In der Experimentalphysik ist eine Doktorarbeit ohne Hilfe und Zusammenarbeit undenkbar. Neben vielen anderen trugen vor allem auch die folgenden Personen zum Gelingen dieser Arbeit bei.

Bei Prof. Dr. Christian Back möchte ich mich einerseits für die interessante Aufgabenstellung bedanken, auch wenn sich das Thema letztendlich weit vom ursprünglich geplanten wegentwickelt hat und so nichts mehr mit Spinphänomenen zu tun hatte. Andererseits möchte ich mich auch für die Betreuung und den angenehmen Umgang miteinander bedanken.

Mein direkter Betreuer Dr. Johann Vancea half mir mit seiner großen Erfahrung bei Experimenten und ermunterte mich meine Ergebnisse und Überlegungen offensiver $\mathrm{zu}$ verteidigen.

Für die angenehme Zusammenarbeit mit meinem Laborkollegen Florian Freund möchte ich mich ebenso bedanken. Das gleiche gilt für "unsere" Diplomanden und Zulassungskandidaten (Michael, Gerhard, Sebastian, Gerhard, Christian).

Unser Lehrstuhltechniker Tobias Stöckl half mit seiner großen Erfahrung bei der Konstruktion von neuen Bauteilen. Besonderer Dank gebührt ihm für seine schnelle Eisatzbereitschaft wenn kurzfristig Bauteile angepasst oder repariert werden mussten.

Allen Mitgliedern des Lehrstuhls Back danke ich für die angenehme Zusammenarbeit und Unterstützung wenn Not am Mann war.

Als Fachmann für mesoskopische Systeme war Prof. Dr. Christoph Strunk für mich immer wieder ein kompetenter Ansprechpartner wenn ich meine Ergebnisse nicht alleine interpretieren konnte. Für die Zeit und die Bereitschaft mir zu helfen möchte ich mich bedanken.

All meine Proben habe ich im Reinraum von Prof. Dr. Dieter Weiss hergestellt. Für den Zugang dazu möchte ich mich bedanken.

Dieter Riedl und Max Simmel aus der Elektronikwerkstatt danke ich für die maßgeschneiderten Elektroniken und vor allem für das Reparieren von Geräten 
die der Hersteller selbst als nicht mehr reparabel einstufte.

Außerdem möchte ich mich bei Manuel, Sebi, Öko, Julia, Sophia und insbesondere Steffi für die angenehme Zeit z.B. beim Teetrinken bedanken, und für die aufbauenden Worte, wenn schon wieder mal eine Probe kaputt war.

Nicht zuletzt möchte ich mich auch bei meinen Eltern für die Unterstützung während meines Studiums und meiner Promotion bedanken. 